\title{
CONFORMAL BOUNDARY OPERATORS, T-CURVATURES, AND CONFORMAL FRACTIONAL LAPLACIANS OF ODD ORDER
}

\author{
A. ROD GOVER AND LAWRENCE J. PETERSON
}

\begin{abstract}
We construct continuously parametrised families of conformally invariant boundary operators on densities. These may also be viewed as conformally covariant boundary operators on functions and generalise to higher orders the first-order conformal Robin operator and an analogous third-order operator of Chang-Qing. Our families include operators of critical order on odd-dimensional boundaries. Combined with the (conformal Laplacian power) GJMS operators, a suitable selection of the boundary operators yields formally self-adjoint elliptic conformal boundary problems. Working on a conformal manifold with boundary, we show that the operators yield odd-order conformally invariant fractional Laplacian pseudo-differential operators. To do this, we use higher-order conformally invariant Dirichlet-to-Neumann constructions. We also find and construct new curvature quantities associated to our new operator families. These have links to the Branson $Q$-curvature and include higher-order generalisations of the mean curvature and the $T$-curvature of Chang-Qing. In the case of the standard conformal hemisphere, the boundary operator construction is particularly simple; the resulting operators provide an elementary construction of families of symmetry breaking intertwinors between the spherical principal series representations of the conformal group of the equator, as studied by Juhl and others. We use our constructions to shed light on some conjectures of Juhl.
\end{abstract}

\section{INTRODUCTION}

Conformally invariant differential operators play a central role in the global geometric analysis of Riemannian, pseudo-Riemannian, and conformal structures $[14,20,55,61$. The nature of conformal geometry means that local issues can also be subtle and difficult. For example, given a proposed leading symbol, a corresponding conformally invariant differential operator may or may not exist, depending on the conformal class. While important open problems remain, nevertheless over the past decades there have been significant advances in our understanding of these issues [22, 33, 40, 41].

By comparison, relatively little is known about natural conformally invariant differential operators along submanifolds. Here we treat an aspect of this gap. Our

Key words and phrases. conformal boundary operators, conformal fractional Laplacians, $T$ curvature, differential intertwinors.

ARG gratefully acknowledges support from the Royal Society of New Zealand via Marsden Grants 13-UOA-018 and 16-UOA-051.

LJP would like to thank the University of Auckland for its hospitality on three separate occasions. 
work is primarily motivated by potential applications to boundary value problems, conformally invariant Dirichlet-to-Neumann constructions (cf. [5, 6, 17, 11, 12, 15, [23, 24]), and related issues linked to geometric PDE, scattering, and the AdS/CFT correspondence of physics [42, 52, 53, 54]. Although we do not treat continuous families of non-local operators here, we believe that our results are complementary to e.g. [7, 12, 15, 42] and that the tools developed here should shed light on such families. The results also give new constructions of families of representation intertwining operators in the sense of Juhl's families [47] and the differential symmetry breaking operators of Kobayashi et al. [50]. See also [18, 28, 49].

In this paper, we construct continuously parametrised families of conformally invariant differential boundary operators on densities. We also construct curvatures associated to these operator families. The new operators may be viewed as conformally covariant boundary operators on functions and for most parameter values generalise to higher orders the well-known first-order conformal Robin operator. We show that some of the operators from the new families combine with the GJMS operators of Graham et al. [41] to yield formally self-adjoint elliptic conformally invariant higher-order Laplacian boundary problems. In conjunction with these problems, we construct higher-order conformally invariant Dirichlet-toNeumann operators that provide realisations of odd-order conformally invariant fractional Laplacian pseudo-differential operators. In the later sections, we work in the setting of general conformally curved manifolds with boundary, but by virtue of the conformal invariance, the results may be applied to conformally compact manifolds and in particular to asymptotically hyperbolic and Poincaré-Einstein manifolds. Earlier work of Branson and the first author in [6] used tractors to construct new families of boundary operators. (We will review tractors in Section 3.2 , below.) In the present article, the approach is again via tractors, but it is simpler, quite different, and most importantly, more effective; we close gaps in [6].

We now explain in more detail the problems treated in this paper, the motivations for these problems, and the results obtained. Throughout our work, $M$ is a manifold of dimension $n, \mathbf{c}$ is a conformal equivalence class of metrics on $M$, and $g$ is a metric in $\mathbf{c}$. The term hypersurface will always refer to a smooth conformally embedded $(n-1)$-dimensional conformal manifold $(\Sigma, \overline{\mathbf{c}})$ in $(M, \mathbf{c})$. In this context, $\overline{\mathbf{c}}$ denotes the conformal equivalence class of the metric on $\Sigma$ induced by any $g \in \mathbf{c}$, and $\bar{g}$ will denote the metric on $\Sigma$ induced by such a $g$. We will often assume that $M$ is initially given as a manifold with boundary, and $\Sigma=\partial M$ is the boundary of $M$. But in this case, for the convenience of treating the local issues, we will assume that we have extended $M$ to a collar neighbourhood of $\Sigma$ (so that $\Sigma$ consists of interior points of $M$ ). Unless we indicate otherwise, we will always assume that $n \geq 3$. For simplicity of discussion, all structures we consider below will be taken to be smooth to infinite order, and we shall restrict to the case of Riemannian signature. These restrictions can be relaxed to a large extent with little change.

The operators in our new families are natural. In Section 2, below, we will discuss the idea of natural differential operators along a hypersurface, as well as the idea of scalar Riemannian hypersurface invariants. We note here, however, that a 
natural differential operator along a hypersurface is a rule that defines a differential operator on a neighbourhood of any hypersurface $(\Sigma, \overline{\mathbf{c}})$ in any ambient conformal $n$-manifold $(M, \mathbf{c})$. In a similar way, a hypersurface invariant is actually a rule which defines an invariant in a neigbourhood of any hypersurface $(\Sigma, \overline{\mathbf{c}})$ of any $(M, \mathbf{c})$. (In both cases, however, we may require $(\Sigma, \overline{\mathbf{c}})$ and $(M, \mathbf{c})$ to meet certain conditions.) The operators in our new families are parametrised by $n$ (in each case in some infinite set $\mathrm{D} \subseteq \mathbb{Z}_{\geq 3}$ ) and a real number $w$. We say that $w$ is a weight. The operators in a given family will always be determined by a single universal symbolic formula, so we will sometimes use the word "operator" to refer to any of our new families of operators or to any other family of operators determined by a single universal symbolic formula. Similar remarks apply to families of hypersurface invariants. Although our main results give operators that, along the hypersurface, are determined by the conformal embedding, the development of these results involves operators that can depend on a choice of metric $g$ within $\mathbf{c}$.

Some of the operators and curvature quantities we construct in this paper are motivated by certain well-known natural differential operators and their associated curvature quantities. One such operator and curvature pair is the conformal Robin operator (of Cherrier [17]) and the mean curvature. To define the conformal Robin operator, we begin by identifying $\Sigma$ with its image submanifold under an embedding $\iota: \Sigma \rightarrow M$. Fixing $g \in \mathbf{c}$ determines a metric $\bar{g} \in \overline{\mathbf{c}}$, and we write $n_{a}$ for the associated unit conormal field to $\Sigma$. This conormal field together with the metric determines a unit normal vector field $n^{a}=g^{a b} n_{b}$. We let $H$ (or sometimes $H^{g}$ ) denote the mean curvature of $\Sigma$. Let $\nabla$ denote the Levi-Civita connection determined by $g$, and let $C^{\infty}(M)$ and $C^{\infty}(\Sigma)$ denote the sets of all smooth realvalued functions on $M$ and $\Sigma$, respectively. The conformal Robin operator is the operator $\delta: C^{\infty}(M) \rightarrow C^{\infty}(\Sigma)$ defined by the composition of $f \mapsto n^{a} \nabla_{a} f-w H f$ with restriction to $C^{\infty}(\Sigma)$. Here $w$ is a real parameter. As before, we say that $w$ is a weight. We define conformal covariance and bidegree in Definition 2.1 below. For all $w \in \mathbb{R}, \delta$ is conformally covariant of bidegree $(-w, 1-w)$.

The operator $\delta$ is a Robin operator because it mixes Dirichlet and Neumann data. Its conformal covariance is important for forming well-posed conformal boundary problems involving the conformal Laplacian (or "Yamabe operator") [6, 17, 23, 45] on the $n$-dimensional interior. Let $w=1-n / 2$, which is the weight selected by the covariance properties of the Yamabe operator, and consider the limiting case in which $n=2$. Then $w=0$, and $\delta$ is just the Neumann operator $n^{a} \nabla_{a}$. The mean curvature $H$ drops out of the formula for $\delta$, but it retains an interesting limiting link with $\delta$ : In this specific case, $H$ is the geodesic curvature and transforms conformally by the rule $H^{\widehat{g}}=e^{-\Upsilon}\left(H^{g}+\delta^{g} \Upsilon\right)$. Here and throughout this paper, $\Upsilon \in C^{\infty}(M)$, and $\widehat{g}=e^{2 \Upsilon} g$.

In connection with the problem of understanding Polyakov-type formulae for conformally covariant elliptic differential operators on compact 4-manifolds with boundary, Chang and Qing discovered a third-order analogue, $P_{3}^{C Q}$, of the conformal Robin operator. (They denoted this operator by $P_{3}$.) See [16]. This operator acts along the boundary of the compact 4-manifold. Chang and Qing showed that $P_{3}^{C Q}$ is naturally associated to a scalar curvature quantity $T=T^{C Q}$ along the 
boundary and that the transformation rule for $T$ under conformal change of metric is $T^{\widehat{g}}=e^{-3 \Upsilon}\left(T^{g}+P_{3, g}^{C Q} \Upsilon\right.$ ). In this and other ways (as explained in Section 7 , below) the relation between $P_{3}^{C Q}$ and $T^{C Q}$ is analogous to the relation between the Neumann operator and the geodesic curvature on a surface with boundary which we described above. By [16], $P_{3}^{C Q}$ is conformally covariant of bidegree $(0,3)$.

In [41, Graham et al. defined a conformally invariant $k$ th power of the Laplacian on a conformal $n$-manifold $(M, \mathbf{c})$. This operator is commonly known as the $G J M S$ operator of order $2 k$, and we will denote it by $P_{2 k}$. It acts on conformal densities, and it is well-defined for all positive integers $k$ within an appropriate range. (We will discuss conformal densities and their weights in Section [3.2, below.) In [4], Branson used $P_{n}$ to define a scalar curvature quantity $Q_{n}$ on a compact Riemannian manifold $(M, g)$ without boundary of even dimension $n \geq 4$. This curvature is called the $Q$-curvature of $(M, g)$. The operator $P_{n}$ may be viewed as a conformally covariant operator $P_{n}^{g}: C^{\infty}(M) \rightarrow C^{\infty}(M)$ of bidegree $(0, n)$. (See Proposition 3.2.) Under conformal change of metric, $Q_{n}$ transforms according to the rule $Q_{n}^{\widehat{g}}=e^{-n \Upsilon}\left(Q_{n}^{g}+P_{n}^{g} \Upsilon\right)$. The pair $(\delta, H)$ in dimension $n=2$ and the Chang-Qing pair $\left(P_{3}^{C Q}, T^{C Q}\right)$ in dimension $n=4$ give, in a certain sense, oddorder boundary analogues of the pair $\left(P_{n}, Q_{n}\right)$. This has generated considerable interest ([11, 13, 47, 53, 54, 59]) and motivates Problem 1.2, below.

The statement of Problem 1.2 involves the notion of the transverse order of a boundary operator along $\Sigma$, as defined in Definition 1.1, below. Definition 1.1 is adapted from the definition of the normal order of a boundary operator given in [6]. Our definition uses a defining function for $\Sigma$ on a neighbourhood $U$ of a point $p \in \Sigma$. Here and below, a such a function is a function $t \in C^{\infty}(U)$ such that $\Sigma \cap U$ is the zero locus of $t$ and $d t$ is nonvanishing on $U$.

Definition 1.1. Let a Riemannian manifold $(M, g)$, a hypersurface $(\Sigma, \bar{g})$ in $(M, g)$, a vector bundle $\mathcal{F}$ over $M$, and a differential operator $B:\left.\mathcal{F} \mapsto \mathcal{F}\right|_{\Sigma}$ be given. Also let $p \in \Sigma$ be given, and suppose that $t$ is any defining function for $\Sigma$ on some neighbourhood $U$ of $p$. For any $m \in \mathbb{Z}_{>0}$, we say that $B$ has transverse order $m$ at $p$ if there is a smooth section $V$ of $\mathcal{F}$ such that $\left.B\left(t^{m} V\right)\right|_{p} \neq 0$ but $\left.B\left(t^{m+1} V^{\prime}\right)\right|_{p}=0$ for all smooth sections $V^{\prime}$ of $\mathcal{F}$. If $\left.B(t V)\right|_{p}=0$ for all smooth sections $V$ of $\mathcal{F}$, then we say that $B$ has transverse order 0 at $p$. Now let $m \in \mathbb{Z}_{\geq 0}$ be given. If $B$ has transverse order $m$ at every $p \in \Sigma$, then we say that $B$ has transverse order $m$. Finally, suppose that $m>0$. If $B$ has transverse order less than $m$ at every $p \in \Sigma$, then we say that $B$ has transverse order less than $m$ or transverse order at most $m-1$.

In the definition here, and also throughout the article, we interpret notation to mean vector bundles or their smooth section spaces according to context. The properties described in this definition are independent of the choice of the defining function $t$. The transverse order at a point $p \in \Sigma$ measures the number of derivatives in directions transverse to $\Sigma$ at $p$. If we say that a natural hypersurface differential operator $B$ has order $m$, order less than $m$, transverse order $m$, or transverse order less than $m$, we mean that the property holds for all hypersurfaces in all possible Riemannian manifolds; for the operator families that we 
deal with in this paper, the presence of these properties may depend on the values of the parameters $w$ and $n$ mentioned above. The conformal Robin operator has transverse order 1 in all dimensions, and $P_{3}^{C Q}$ has transverse order 3 .

In the statement of Problem 1.2 that follows, and throughout this paper, differential operators will act on everything to their right, unless parentheses indicate otherwise. In addition, we will always indicate multiplication operators by juxtaposition. For example, let differential operators $O p_{1}, O p_{2}: C^{\infty}(M) \rightarrow C^{\infty}(M)$ and functions $f_{1}, f_{2} \in C^{\infty}(M)$ be given. Then $O p_{1} f_{1} O p_{2} f_{2}$ denotes $O p_{1}$ acting on the product of $f_{1}$ and $O p_{2} f_{2}$. All differential operators in this paper will be linear.

We may now state one of the main problems of this paper.

Problem 1.2. For some $K \in \mathbb{Z}_{>0}$, construct a family of natural hypersurface operators $P_{w, K}^{g}: C^{\infty}(M) \rightarrow C^{\infty}(\Sigma)$ parametrised by the dimension $n$ and a real parameter $w$ such that the following properties hold: (1) For all $w \in \mathbb{R}, P_{w, K}^{g}$ has order at most $K$. There is a small finite set $E$ such that for all $w \in \mathbb{R} \backslash E, P_{w, K}^{g}$ has order and transverse order $K$. The set $E$ may depend on $n$. (2) For all $f \in C^{\infty}(M)$ and all $w \in \mathbb{R}, P_{w, K}^{\widehat{g}} f=e^{-(K-w) \Upsilon} P_{w, K}^{g} e^{-w \Upsilon} f$. (3) The family $P_{w, K}^{g}$ determines a scalar curvature quantity $Q$ along $\Sigma$ with the property that $Q^{\widehat{g}}=e^{-K \Upsilon}\left(Q^{g}+P_{0, K}^{g} \Upsilon\right)$. This scalar curvature quantity is a local scalar Riemannian hypersurface invariant.

One of our main objectives will be to find solutions to Problem 1.2 in which the set $E$ of exceptional weights is as small as possible or solutions in which some specific undesirable weight (such as $w=0$ ) is absent from $E$.

We will be especially interested in higher-order generalisations of the conformal (Cherrier-)Robin operator and the Chang-Qing operator. The Chang-Qing operator has order and transverse order $n-1=3$, the dimension of $\Sigma$. Similarly, in the limiting case in which $n=2$, the conformal Robin operator likewise has order and transverse order $n-1$. In general even dimensions $n$, establishing the existence of conformal boundary operators of order and transverse order $n-1$ is rather delicate. Such operators sit in a special position that is in part analogous to the place of the dimension-order GJMS operators of [41]. Thus, in the setting of Problem 1.2, if $n$ is even, we will say that a boundary operator of order and transverse order $n-1$ is a critical operator and that $n-1$ is the critical order. Paramount in our considerations here is finding a solution to Problem 1.2 that includes such operators as part of a continuous family. For emphasis we state the following special case of Problem 1.2.

Problem 1.3. In Problem 1.2, suppose that $n$ is even and $K=n-1$. Find a solution such that $P_{0, K}^{g}$ is a critical operator.

In this paper, we construct families of operators which solve Problem 1.3 in general even dimensions $n \geq 4$. We do this by first constructing three families of operators which solve Problem 1.2. Let $K$ be as in Problem 1.2. Then for all $K \in \mathbb{Z}_{>0}$, the operator family $\delta_{K}^{0}$ of Theorem 5.12 solves Problem 1.2 in the simplest setting, namely the case of conformally flat metrics. (Throughout this paper, conformally flat means what is sometimes referred to as "locally conformally flat", that is, the Weyl and Cotton tensors both vanish. Also, note that we suppress 
the $g$ and the $w$ from the operator notation.) The $\delta_{K}^{0}$ family is defined in all dimensions $n \geq 3$. Next, for all $K \in \mathbb{Z}_{>0}$, the operators $\delta_{J, k}$ of Theorem [5.16, with $J+k=K$, are defined for general Riemannian conformal structures in suitable dimensions and for conformally flat structures in all dimensions $n \geq 3$. Similarly, for all $K \in \mathbb{Z}_{>0}$, the operators $\delta_{K}$ of Lemma 5.4 are defined on general Riemannian conformal structures in all dimensions $n \geq 3$. The families $\delta_{J, k}$ and $\delta_{K}$ solve Problem 1.2.

The $\delta_{K}^{0}$ operator family solves Problem 1.2 on the standard conformal hemisphere, and in this case, the operators $\delta_{K}^{0}$ provide families of symmetry breaking intertwinors between the spherical principal series representations of the conformal group of the equator, as studied by Juhl and others [18, 47, 50]. There is some appeal in this picture, as here the symmetry breaking (the reduction of the conformal group of the sphere to a subgroup preserving the closed hemisphere) is manifestly governed by the normal tractor. By using the tools in, for example, [34], one may develop generalisations of the $\delta_{K}^{0}$ and $\delta_{J, k}$ families which treat differential forms and other tensors. Such generalisations should be of some interest to the more general intertwinor programme as in [28, 48, but we do not investigate this idea in this paper.

As part of a construction of a family of conformally invariant higher-order Dirichlet-to-Neumann operators, Branson and the first author discover and construct a family $\delta_{K}^{B G}$ of conformally invariant hypersurface operators in Theorem 5.1 of [6]. Although used at a discrete set of weights in [6], by construction the operators are available on any density bundle, and in the interesting work [47], Juhl studies the resulting continuously parametrised families of boundary operators (as well as so-called "residue families") and considers some problems linked to those studied here; see for example [47, Section 1.10 and Section 6.21]. Unfortunately, as evident in [6, Theorem 5.1] and its proof, the $\delta_{K}^{B G}$ family does not provide an answer to Problem 1.3. In [43], Grant constructs a modification $\delta_{3}^{G}$ of $\delta_{3}^{B G}$ which solves Problem 1.3 in the dimension $n=4$ case. (See also Stafford [57] and Case [11.) However, Grant's work was specific to third-order operators, and the problem of finding higher-dimensional analogues of the Chang-Qing pair $\left(P_{3}^{C Q}, T^{C Q}\right)$ has, to our knowledge, remained open up to now.

For every even integer $n \geq 4$, the $\delta_{K}^{0}$ family includes an operator which solves Problem 1.3 for conformally flat Riemannian conformal manifolds of dimension $n$, and the $\delta_{J, k}$ family contains a solution to this same problem for general Riemannian conformal manifolds of dimension $n$. See Theorem 7.10, below.

In Section 6.1, the operators $\delta_{J, k}$ and $\delta_{K}$ are used to set up conformally invariant elliptic boundary problems for the GJMS operators and then to construct non-local operators. The first main result is Theorem 6.3, which, for any GJMS operator $P_{2 k}$, describes a corresponding conformally invariant boundary system $B$ such that the boundary value problem $\left(P_{2 k}, B\right)$ is formally self-adjoint. The boundary system $B$ is given by (50). Lemma 6.5 then shows that the boundary problem $\left(P_{2 k}, B\right)$ satisfies the Lopatinski-Shapiro condition. The pair $\left(P_{2 k}, B\right)$ is properly elliptic, and in Theorem 6.7, we use this fact, together with the other properties of the pair, to construct Dirichet-to-Neumann operators. The statement of Theorem 6.7 is too 
technical to be given in this introduction, but we may easily state two corollaries of the theorem here. In these corollaries, $\overline{\mathcal{E}}[w]$ denotes the bundle of conformal densities of weight $w$ associated to $(\Sigma, \overline{\mathbf{c}})$; here $w$ is any given real number. The first corollary follows from the case $k=n / 2$ ( $n$ even) of Theorem 6.7 and Lemma 6.1.

Corollary 1.4. Let a compact Riemannian conformal manifold with boundary $(M, \Sigma, \mathbf{c})$ of even dimension $n \geq 4$ be given. Let $B$ be as in (50), and suppose that $\left(P_{n}, B\right)$ has trivial kernel. Then for $2 m=1,3,5, \ldots, n-1$, Theorem 6.7 yields conformally invariant non-local operators

$$
P_{2 m}^{T, n / 2}: \overline{\mathcal{E}}\left[m-\frac{n-1}{2}\right] \rightarrow \overline{\mathcal{E}}\left[-m-\frac{n-1}{2}\right]
$$

which have leading term $(-\bar{\Delta})^{m}$.

Corollary 1.4 produces a critical-order (i.e. order $n-1$ ) fraction Laplacian as the case $2 m=n-1$. Such an operator was missing from the results of [6].

The next corollary also follows from Theorem 6.7 and Lemma 6.1.

Corollary 1.5. Let a compact Riemannian conformal manifold with boundary $(M, \Sigma, \mathbf{c})$ of dimension $n \geq 3$ be given. Also let $k \in \mathbb{Z}_{>0}$ be given, and suppose that (1) $n$ is odd, or (2) $n$ is even and $k \leq n / 2$, or (3) $(M, \mathbf{c})$ is conformally flat. Finally, let $B$ be as in (50), and suppose that $\left(P_{2 k}, B\right)$ has trivial kernel. Then for $2 m=1,3,5, \ldots, 2 k-1$, Theorem 6.7 yields conformally invariant non-local operators

which have leading term $(-\bar{\Delta})^{m}$.

$$
P_{2 m}^{T, k}: \overline{\mathcal{E}}\left[m-\frac{n-1}{2}\right] \rightarrow \overline{\mathcal{E}}\left[-m-\frac{n-1}{2}\right]
$$

For each solution $P_{w, K}^{g}$ to Problem 1.2, we will say that the associated scalar curvature quantity $Q$ is a $Q$-type curvature. This $Q$-type curvature has certain properties analogous to those of Branson's $Q$-curvature. However, for any dimension $n$ and any $K \in \mathbb{Z}_{>0}$, if a solution $P_{0, K}^{g}$ has transverse order $K$, then we will say that the $Q$-type curvature associated to $P_{w, K}^{g}$ is a $T$-curvature of order $K$ and that $\left(P_{0, K}^{g}, Q^{g}\right)$ is a $T$-curvature pair of this order. We often let $T^{g}$ denote $Q^{g}$ in this case. In Section 7, we will see that for any given even dimension $n \geq 4$, there are $T$-curvature pairs of all orders. Specifically, we will establish the following theorem:

Theorem 1.6. Let $n_{0} \in \mathbb{Z}_{\geq 4}$ be given, and suppose that $n_{0}$ is even. Then in dimension $n=n_{0}$, there are canonical $T$-curvature pairs

$$
\begin{aligned}
& \left(\delta_{1}, T_{1}^{g}\right),\left(\delta_{2}, T_{2}^{g}\right), \ldots,\left(\delta_{n_{0} / 2}, T_{n_{0} / 2}^{g}\right),\left(\delta_{1, n_{0} / 2}, T_{1+n_{0} / 2}^{g}\right), \ldots, \\
& \quad\left(\delta_{\left(n_{0}-2\right) / 2, n_{0} / 2}, T_{n_{0}-1}^{g}\right),\left(\delta_{n_{0}}, T_{n_{0}}^{g}\right),\left(\delta_{n_{0}+1}, T_{n_{0}+1}^{g}\right), \ldots
\end{aligned}
$$

of orders $1,2,3, \ldots$, respectively.

Let $g \in \mathbf{c}$ be given. Proposition [7.15, below, gives conditions which ensure that for all $m \in \mathbb{Z}_{\geq 2}$, there is a metric $\widehat{g} \in \mathbf{c}$ which induces $\bar{g}$ and which satisfies $T_{1}^{\widehat{g}}=T_{2}^{\widehat{g}}=\cdots=T_{m}^{\widehat{g}}=0$ along $\Sigma$. Under these same conditions, $T_{1}^{\widehat{g}}=H^{\widehat{g}} ;$ this 
will become clear later. So under these conditions, the hypersurface $\Sigma$ is minimal for the metric $\widehat{g}$ in the sense that $H^{\widehat{g}}=0$ along $\Sigma$, and $\Sigma$ also satisfies the related higher-order condition $T_{2}^{\widehat{g}}=\cdots=T_{m}^{\widehat{g}}=0$ along $\Sigma$.

We have computed explicit symbolic symbolic formulae for our new operator families and their $Q$-type curvatures in certain cases. One such formula is the following formula for the $Q$-type curvature of the $\delta_{1,2}$ family:

$$
3 n^{a} \nabla_{a} \mathrm{~J}-(n-2) n^{a} n^{b} n^{c} \nabla_{a} \mathrm{P}_{b c}+6 H \mathrm{~J}-6(n-2) H n^{a} n^{b} \mathrm{P}_{a b}+2(n-2) H^{3} .
$$

Here $\mathrm{P}_{a b}$ is the Schouten tensor, and $\mathrm{J}=\mathrm{P}_{a}{ }^{a}$. (We define the Schouten tensor in Section 3, below.) We give explicit formulae for $\delta_{1,2}$ and a few of our other operator families and their curvatures in Section 8 , below. Some of these formulae are valid only in certain dimensions, as explained in that section.

Our operator and curvature constructions use the Fefferman-Graham ambient metric of [25, 26], its link to tractors [9], and the tractor construction of the GJMS operators developed in [35]. We will often work with symbolic formulae which are polynomial in the parameter $w$ of Problem 1.2 and rational in the dimension $n$. As a consequence, our proofs will often use polynomial continuation in $w$ and rational continuation in $n$. To treat these notions, we develop some tools and results in Section 4, below.

\section{Conformally COVARiant operators Along a hypersurface}

In this section, we work with arbitrary Riemannian metrics. We will generally employ Penrose's abstract index notation. (If no ambiguity will occur, however, we will sometimes omit indices from tensors.) We shall write $\mathcal{E}^{a}$ to denote the space of sections of the tangent bundle $T M$ over $M$, and $\mathcal{E}_{a}$ for the space of sections of the cotangent bundle $T^{*} M$. (In fact, we will often use the same symbols for the bundles themselves.) We write $\mathcal{E}$ for the space of real-valued functions on $M$ (or for the trivial bundle $M \times \mathbb{R}$ ). All functions, vector bundles, and sections of vector bundles will be assumed to be smooth, meaning $C^{\infty}$. An index which appears twice, once raised and once lowered, indicates a contraction. The metric $g_{a b}$ and its inverse $g^{a b}$ enable the identification of $\mathcal{E}^{a}$ and $\mathcal{E}_{a}$, and we indicate this by raising and lowering indices in the usual way. In Section 3 , below, we will discuss the Riemannian and Weyl curvature tensors and the Ricci and Schouten tensors; throughout much of this paper, the term "Riemannian curvature tensor" will include these tensors and the traces of the Ricci and Schouten tensors.

Let an embedded hypersurface $\Sigma$ of $M$ be given, and suppose that $M$ and $\Sigma$ are both orientable. We will usually work locally and assume that $\Sigma$ is the zero locus $\mathcal{Z}(t)$ of a defining function $t$, so the orientability assumptions will usually not impose any restriction. We will need to consider geometric quantities determined on $\Sigma$. Rather than deal with the awkwardness of fields and quantities which are defined only along $\Sigma$, we will define extensions of these into a neighbourhood of $\Sigma$; we emphasise that our final results will not depend on the choice of these extensions. We will calculate in a neighbourhood on which $d t$ is nowhere zero, and 
we define

$$
n_{a}:=\frac{d t}{|d t|_{g}}
$$

in this neighbourhood. The level sets of $t$ define a foliation $\Sigma_{t}$, with $\Sigma=\Sigma_{0}$, such that $n_{a}$ gives the unit conormal field along each leaf $t=$ constant.

For operators on functions, the notion of naturality along a hypersurface is an obvious adaptation of the usual notion from Riemannian geometry (see, e.g., [2, 58]). A natural differential operator along a hypersurface is a differential operator which, in a neighbourhood of the hypersurface, may be expressed by a universal symbolic formula which is polynomial in the Levi-Civita connection $\nabla$ of $(M, g)$ and has tensor-valued pre-invariants as coefficients. We refer the reader to Section 2.4 of [37] for the definitions of scalar- and tensor-valued Riemannian hypersurface pre-invariants and invariants. In this paper, any family of natural operators will always be given by the same universal symbolic formula for all possible conformal manifolds $(M, \mathbf{c})$; a similar remark applies to families of invariants. The symbolic formula for a family of such operators or invariants will be a polynomial in the conormal field $n_{a}$, the Riemannian metric $g_{a b}$, its inverse $g^{a b}$, the Riemannian curvature tensor $R_{a b}{ }^{c} d$, the mean curvature $H$, and the Levi-Civita connection $\nabla_{a}$. (We give examples of symbolic formulae for operators and invariants at various points in this paper. Tractors, the trace-free part of the second fundamental form, and various operators will appear in some of these formulae, but it will always be possible to expand these formulae and write them as polynomials of the above type.) The coefficients in the polynomial formula for a family of operators or invariants will be real functions of the dimension $n$ and the weight parameter $w$ that we discussed in Section 1, above. This real function will be polynomial in $w$ and rational in $n$. In the case of an invariant, $w$ will be absent from the formula. For most of the universal formulae that we work with in this paper, we may assume that $\nabla_{a}$ never explicitly hits $n_{b}$ or $H$.

In Section 3.2, below, we will incorporate conformal densities into our operators and invariants. The above definitions of natural operators and hypersurface invariants will extend to this situation in the obvious way. Our work will also require the notion of natural differential operators between sections of tractor bundles. As we will see in Section 3.2, below, a tractor bundle is a finite-dimensional vector bundle over $(M, \mathbf{c})$. We will see that for each $g \in \mathbf{c}$, a tractor bundle decomposes into a direct sum of tensor bundles. Naturality of a differential operator between sections of tractor bundles will mean naturality with respect to any such decomposition. Our earlier discussion concerning families of operators and invariants extends to our work with densities and tractors in an obvious way.

Now suppose that we choose an orientation for $\Sigma$. Then on $\Sigma$, hypersurface invariants and natural differential operators along $\Sigma$ are independent of the choice of the defining function $t$, because we insist that $d t /|d t|_{g}$ be consistent with the orientation. Such invariants and operators need not be uniquely determined off of $\Sigma$. Let $\mathcal{F}$ denote $\mathcal{E}$ or the set of all smooth sections of any power of the tractor bundle over $M$, and let $V \in \mathcal{F}$ be given. Also let a natural hypersurface differential 
operator $O p:\left.\mathcal{F} \rightarrow \mathcal{F}\right|_{\Sigma}$ be given. Then $O p V$ will usually denote the restriction of $O p V$ to $\Sigma$, but we will sometimes indicate the restriction explicitly by writing $\left.O p V\right|_{\Sigma}$. We will sometimes write $O p: \mathcal{F} \rightarrow \mathcal{F}$ instead of $O p:\left.\mathcal{F} \rightarrow \mathcal{F}\right|_{\Sigma}$.

One example of a scalar hypersurface invariant is the mean curvature $H=$ $(1 /(n-1)) \bar{g}^{a b} L_{a b}$. Here $L_{a b}$ is the second fundamental form, and $\bar{g}^{a b}$ is the hypersurface metric determined by $g$ as in Section 3.1, below. Similarly, $L_{a b}$ is a tensor-valued hypersurface invariant, and $|L|^{2}=L_{a b} L^{a b}:=\bar{g}^{a c} \bar{g}^{b d} L_{a b} L_{c d}$ is another scalar invariant.

For any $w \in \mathbb{R}$, a scalar Riemannian hypersurface invariant $K$ determines a conformal invariant of weight $w$ if it satisfies the conformal covariance relation $K\left(e^{2 \Upsilon} g, t\right)=e^{w \Upsilon_{\Sigma}} K(g, t)$ for all $\Upsilon \in \mathcal{E}$. Here $\Upsilon_{\Sigma}$ is the pullback of $\Upsilon$ to the hypersurface $\Sigma$. Thus $K$ determines a homogeneous function on $\mathcal{Q}$, the bundle of conformal metrics. This function represents an invariant conformal density of weight $w$.

Definition 2.1. We say that a natural differential operator $P^{g}: \mathcal{E} \rightarrow \mathcal{E}$ is conformally covariant of bidegree $\left(w_{1}, w_{2}\right)$ in $\mathbb{R}^{2}$ if $P^{\widehat{g}} V=e^{-w_{2} \Upsilon} P^{g} e^{w_{1} \Upsilon} V$ for all $g$ and for all $\Upsilon$ and $V$ in $\mathcal{E}$. This definition extends to hypersurface operators and operators on tractors in the obvious way.

In Section 3.3, we will replace conformal covariance with the equivalent notion of conformal invariance. This will simplify the discussion and calculations. Our aim will be to construct special natural conformally invariant operators; there will be no attempt to classify operators. The strategy is to build these in such a way that, by construction, they satisfy the naturality conditions. Achieving conformal invariance, although more subtle, will eventually be seen to yield to the same approach.

Before continuing, we consider two examples of conformally covariant natural operators. For the conformal Robin operator $\delta:\left.\mathcal{E} \rightarrow \mathcal{E}\right|_{\Sigma}$, naturality is evident from the formula

$$
\delta f:=n^{a} \nabla_{a} f-w H f .
$$

We will also let $\delta_{1}$ or $\delta_{1, g, w}$ denote the conformal Robin operator. In any dimension $n \geq 2$, the mean curvature $H$ satisfies the conformal transformation rule $H_{\widehat{\mathrm{g}}}=$ $e^{-\Upsilon}\left(H_{g}+\delta_{1, g, 0} \Upsilon\right)$. From this it follows that for any $w \in \mathbb{R}$, the conformal Robin operator is conformally covariant of bidegree $(-w, 1-w)$.

A second-order analogue of $\delta_{1}$ is the operator $\delta_{2}$ (also denoted by $\delta_{2, g, w}$ ) given by the formula

(4)

$$
\begin{aligned}
& \delta_{2} f= \\
& \quad-(\Delta+w \mathrm{~J}) f+(n+2 w-2) n^{a} n^{b} \nabla_{a} \nabla_{b} f-2(w-1)(n+2 w-2) H n^{a} \nabla_{a} f \\
& \quad+(w-1) w(n+2 w-2) H^{2} f+w(n+2 w-2) n^{a} n^{b} \mathrm{P}_{a b} f .
\end{aligned}
$$

Here $\Delta=\nabla_{i} \nabla^{i}$ and $f \in \mathcal{E}$. In Section 3 , below, we will see that $\mathrm{P}_{a b}$ and $\mathrm{J}$ are Riemannian invariants. From this it will follow that $\delta_{2}$ is manifestly natural, since each object in the formula for $\delta_{2}$ is determined by the data of the ambient Riemannian structure on $M$ and the embedding. No other information is involved. 
The normal vector field $n^{a}$ appears in the formula, but along $\Sigma$, the value of $\delta_{2} f$ is independent of the extension of $n^{a}$ off of $\Sigma$. Consideration of the tractor formula for $\delta_{2}$ in Section 5.1 will show that if $w$ is any real number, then $\delta_{2, g, w}$ is conformally covariant of bidegree $(-w,-w+2)$. If $w=1-n / 2$, then $\delta_{2}=-\square$, where $\square$ is as defined in (16), below. In this case, $\square$ is the Yamabe operator for $(M, \mathbf{c})$. In Section 8.1, we will relate $\delta_{2}$ to the intrinsic Yamabe operator on $\Sigma$ and to a secondorder hypersurface operator of [43]. Finally, note that $\delta_{2}$ is considerably more complex than $\delta_{1}$. It is easily seen that there is exponential growth in complexity as order increases. A naïve approach to conformal submanifolds will therefore not suffice.

\section{Conformal Geometry and hypersurfaces}

Let $\nabla_{a}$ denote the Levi-Civita connection on a Riemannian manifold $(M, g)$ of dimension $n \geq 2$. The Riemannian curvature tensor $R$ on $(M, g)$ is defined by

$$
\mathrm{R}(X, Y) Z=\nabla_{X} \nabla_{Y} Z-\nabla_{Y} \nabla_{X} Z-\nabla_{[X, Y]} Z .
$$

Here $X, Y$, and $Z$ are arbitrary vector fields. In abstract index notation, $R$ is denoted by $R_{a b}{ }^{c}$, and $\mathrm{R}(X, Y) Z$ is $X^{a} Y^{b} Z^{d} R_{a b}{ }^{c} d$. In dimensions $n \geq 3$ this can be decomposed into the totally trace-free Weyl curvature $C_{a b c d}$ and the symmetric Schouten tensor $\mathrm{P}_{a b}$ according to

$$
R_{a b c d}=C_{a b c d}+2 g_{c[a} \mathrm{P}_{b] d}+2 g_{d[b} \mathrm{P}_{a] c},
$$

where $[\cdots]$ indicates antisymmetrisation over the enclosed indices. In (5) , $\mathrm{P}_{a b}$ is a trace modification of the Ricci tensor given by

$$
\mathrm{Rc}_{a b}=(n-2) \mathrm{P}_{a b}+\mathrm{Jg}_{a b} .
$$

Here $\mathrm{Rc}_{a b}=R_{c a b}{ }^{c}$, and $\mathrm{J}=\mathrm{P}_{a}{ }^{a}$. In dimensions 2 and 3 the Weyl tensor $C_{a b c d}$ vanishes identically by dint of its symmetries. In dimension 2, (6) does not define a Schouten tensor in the sense we require in this work. By adding additional structure (a Möbius structure [8]), however, one may define a Schouten tensor in dimension 2 in such a way that that it has conformal properties similar to the Schouten tensor in higher dimensions.

3.1. Riemannian hypersurfaces. In this subsection, we discuss covariant derivatives and various structures on $\Sigma$ and relate them to covariant derivatives and structures on $M$. These ideas will facilitate the understanding of some of the example formulae in Section 8, below. This material is standard and appears in such sources as [56], Chapter 1, but we develop it here to set notation fit with our current approach via defining functions, cf. [19, 37, 43, 57].

To begin, let $\Sigma$ denote a hypersurface in $(M, g)$ as in Section 1, and let $t$ denote any local defining function for $\Sigma$, as in Section 2. We are concerned with local theory here, so without loss of generality, we may assume that $d t$ is nowhere zero. Let $n_{a}$ be as in (2). Recall that the level sets of $t$ define a foliation $\Sigma_{t}$, with $\Sigma=\Sigma_{0}$. For each leaf $t=t_{0}$, the embedding $\iota: \Sigma_{t_{0}} \rightarrow M$ induces an injective bundle map $\iota_{*}: T \Sigma_{t_{0}} \rightarrow T M$, and we shall simply identify $T \Sigma_{t_{0}}$ with its image. Dually, $T^{*} \Sigma_{t_{0}}$ is naturally a quotient of $T^{*} M$. The bundle epimorphism $T^{*} M \rightarrow T^{*} \Sigma_{t_{0}}$ is split 
by the metric, and so $T^{*} \Sigma_{t_{0}}$ is naturally identified with the subbundle of $T^{*} M$ consisting of 1 -forms orthogonal to the conormal bundle to $\Sigma_{t_{0}}$. We shall thus use the same abstract indices for $T \Sigma_{t_{0}}$ as we do for $T M$. We will, however, use a bar to denote objects intrinsic to $\Sigma$ or to any leaf of the foliation. Thus $\overline{\mathcal{E}}^{a}$ (resp. $\overline{\mathcal{E}}_{a}$ ) will denote the subbundle of $\mathcal{E}^{a}$ defined by the foliation (resp. the subbundle of 1-forms annihilating $n^{a}$ ), the spaces of smooth sections of these, or the restrictions of these objects to a leaf $\Sigma_{t_{0}}$ (for any $t_{0} \in \mathbb{R}$ where the foliation is defined). For example, $u_{a} \in \mathcal{E}_{a}$ is a section of $\overline{\mathcal{E}}_{a}$ if and only if $u_{a} n^{a}=0$. In particular, this applies along $\Sigma=\Sigma_{0}$, where our interest really lies, and any section of $\overline{\mathcal{E}}^{a}$ (resp. $\overline{\mathcal{E}}_{a}$ ) along $\Sigma$ will be assumed to be the restriction to $\Sigma$ of a section of $\overline{\mathcal{E}}^{a}$ (resp. $\overline{\mathcal{E}}_{a}$ ) defined in a neighbourhood of $\Sigma$. This idea will be extended to tensor powers in an obvious way with little further mention.

Next, let $\Pi_{a}{ }^{b}:=\delta_{a}{ }^{b}-n_{a} n^{b}$. As a section of $\operatorname{End}(T M)$, this defines projections $T M \rightarrow T \Sigma_{t_{0}}$ and $T^{*} M \rightarrow T^{*} \Sigma_{t_{0}}$ in the obvious way. Thus, for example, the formula $\bar{g}_{a b}:=g_{a b}-n_{a} n_{b}$ defines a symmetric 2-tensor that restricts to give an induced intrinsic metric along any $\Sigma_{t_{0}}$. Similarly $\bar{g}^{a b}=g^{a b}-n^{a} n^{b}$, which is consistent with raising indices using the ambient metric $g^{a b}$. We write $\bar{\nabla}$ for the corresponding intrinsic Levi-Civita connection along the leaves, and $\overline{\mathrm{R}}$ denotes the corresponding Riemannian curvature. Although we shall be finally interested in these quantities only along $\Sigma=\Sigma_{0}$, it is convenient to have fixed an extension off $\Sigma$ in this way; this is consistent with our treatment of vector fields and tensors in general, as discussed above. They depend smoothly on points in the foliated neighbourhood of $\Sigma$.

Along $\Sigma$, and indeed along each leaf $\Sigma_{t_{0}}$, the second fundamental form of the embedding, $L_{a b}$, is given by the formula $L_{a b}=\Pi_{a}{ }^{c} \nabla_{c} n_{b}$. (Note that the sign of $L$ here differs from that of many sources, including [56].) By the Weingarten equations $L_{a b}$ is symmetric. An easy exercise shows that $n^{a} L_{a b}=0$, so $L$ defines a smooth section of $\overline{\mathcal{E}}_{(a b)}$, the second symmetric power of $\overline{\mathcal{E}}_{a}$. Let $V^{a}, T^{b} \in \overline{\mathcal{E}}^{a}$ be given. Then $L_{a b} V^{a} T^{b}=-g\left(n^{a}, \nabla_{V} T\right)$ along $\Sigma$, again by the Weingarten equations. Thus if $d t$ is compatible with a given orientation on $\Sigma$, then $L_{a b}$, and hence also the mean curvature $H$, are independent of the choice of the defining function $t$. The metric trace-free part of $L$, denoted by $\stackrel{o}{L}$, is given by $\stackrel{o}{L}_{a b}=L_{a b}-H \bar{g}_{a b}$.

An easy computation shows that $H=(1 /(n-1)) \nabla_{a} n^{a}$. Hypotheses 4.1, below, will refer to a modified mean curvature tensor $G$ given by $G=\nabla_{a} n^{a}$. The purpose of defining $G$ in this way is to obtain a curvature given by a symbolic formula which involves $\nabla_{a}$ and $n^{a}$ but not the dimension $n$.

It is easily verified that $\bar{\nabla}$ is related to the ambient Levi-Civita connection $\nabla$ by the Gauss formula

$$
\bar{\nabla}_{a} V^{b}=\Pi_{a}^{c} \nabla_{c} V^{b}+n^{b} V^{c} L_{a c},
$$

where $V^{a} \in \overline{\mathcal{E}}^{a}$; in particular $\bar{\nabla}_{a} V^{b}=\Pi_{a}{ }^{c} \prod_{d}{ }^{b} \nabla_{c} V^{d}$. From this follows the classical Gauss equation, which we give in our current notation:

Proposition 3.1. Let $\bar{R}_{a b}{ }^{c}$ denote the intrinsic Riemannian curvature tensor. Then

$$
\bar{R}_{a b c d}=\Pi_{a}^{i} \Pi_{b}^{j} \Pi_{c}^{k} \Pi_{d}^{l} R_{i j k l}+L_{a c} L_{b d}-L_{a d} L_{b c} .
$$


3.2. Conformal structures. A conformal geometry is a manifold of dimension at least 2 equipped with a conformal structure, i.e. an equivalence class $\mathbf{c}$ of Riemannian metrics such that if $g, \widehat{g} \in \mathbf{c}$, then $\widehat{g}=e^{2 \Upsilon} g$ for some $\Upsilon \in \mathcal{E}$. Our operator constructions will require several results and techniques from conformal geometry. For further details see [3, 9, 35], or [19] for a recent overview.

We begin by interpreting a conformal structure c as a ray subbundle $\mathcal{Q} \subseteq$ $S^{2} T^{*} M$ whose fibre over $x \in M$ consists of the set of all metrics at $x$ which are conformally related to some given metric $g$ at the point $x$. The principal bundle $\pi: \mathcal{Q} \rightarrow M$ has structure group $\mathbb{R}_{>0}$, so for any $w \in \mathbb{R}$, the representation $\mathbb{R}_{>0} \ni x \mapsto x^{-w / 2} \in \operatorname{End}(\mathbb{R})$ induces a natural (oriented) line bundle on $M$ that we term the bundle of conformal densities of weight $w$. We let $\mathcal{E}[w]$ denote the space of sections of this bundle or the bundle itself. There is a tautological section $\boldsymbol{g}_{a b}$ of $S^{2} T^{*} M \otimes \mathcal{E}[2]$ that is termed the conformal metric. Similarly, $\mathbf{g}^{-1}$ is a section $\mathbf{g}^{a b}$ of $S^{2} T M \otimes \mathcal{E}[-2]$. Henceforth, $\mathbf{g}_{a b}$ and $\mathbf{g}^{a b}$ will be the default objects that will be used to identify $T M$ with $T^{*} M \otimes \mathcal{E}[2]$ and to raise and lower indices associated to these bundles (even when a metric $g \in \mathbf{c}$ has been chosen). As a consequence, tensors, hypersurface invariants, and natural differential operators will now carry weights. For example, if $R_{a b}{ }^{c}{ }_{d}$ is the Riemannian curvature tensor associated to a metric $g \in \mathbf{c}$, then $R_{a b}{ }^{c}$ will have weight 0 . Similarly, $R_{a b c d}$ will denote $\boldsymbol{g}_{c e} R_{a b}{ }^{e} d$, and this tensor will have weight 2 . The weights of other such curvature tensors will be evident from their definitions. We will assign weights to $n_{a}, L_{a b}, H$, and $\stackrel{o}{L}_{a b}$ in Section 3.3. If $P$ is a natural differential operator acting between densities, then for some $c \in \mathbb{R}$ and all conformal manifolds $(M, \mathbf{c})$ (or all conformally flat conformal manifolds $(M, \mathbf{c}))$ of appropriate dimensions, $P$ will map $\mathcal{E}[w]$ to $\mathcal{E}[w-c]$. For example, for any $f \in \mathcal{E}[w]$, we have $\Delta f=\mathbf{g}^{a b} \nabla_{a} \nabla_{b} f \in \mathcal{E}[w-2]$.

A metric $g \in \mathbf{c}$ is equivalent to a positive section $\xi^{g}$ of $\mathcal{E}[1]$ via the relation $g_{a b}=\left(\xi^{g}\right)^{-2} \boldsymbol{g}_{a b}$. We say that $\xi^{g}$ is the scale density associated to $g$. Let $w \in \mathbb{R}$ and a section $\sigma$ of $\mathcal{E}[w]$ be given. We may write $\sigma=\left(\xi^{g}\right)^{w} f$ for some $f \in \mathcal{E}$. It is easily verified that the Levi-Civita connection $\nabla$, of $g$, acting on $\mathcal{E}[w]$, is the connection mapping $\sigma$ to $\left(\xi^{g}\right)^{w} d f$, where $d$ is the exterior derivative. Let $\mathcal{E}_{b}[w]=\mathcal{E}_{b} \otimes \mathcal{E}[w]$, and let $\widehat{\nabla}$ denote the Levi-Civita connection associated to the metric $\widehat{g}=e^{2 \Upsilon} g$. Then for all $\mu_{b} \in \mathcal{E}_{b}[w]$,

$$
\widehat{\nabla}_{a} \mu_{b}=\nabla_{a} \mu_{b}+(w-1) \Upsilon_{a} \mu_{b}-\Upsilon_{b} \mu_{a}+\mathbf{g}_{a b} \Upsilon^{c} \mu_{c}
$$

where $\Upsilon_{a}=\nabla_{a} \Upsilon$.

On a general conformal manifold there is no distinguished connection on $T M$. There is, however, a canonical conformally invariant connection on a slightly larger bundle, and this is called the (conformal) tractor connection 3 . It is linked, and equivalent to, the normal conformal Cartan connection of Elie Cartan [10]. On a conformal manifold $(M, \mathbf{c})$ of dimension $n \geq 3$, let $\mathcal{T}$ (or $\mathcal{T}^{A}$ as the abstract index notation) denote the (standard) tractor bundle. This bundle is a canonical rank $n+2$ vector bundle equipped with the canonical (normal) tractor connection $\nabla^{\mathcal{T}}$. This connection is conformally invariant. We usually write $\nabla$ instead of $\nabla^{\mathcal{T}}$. We let $\mathcal{T}^{\Phi}$ denote any tensor power of $\mathcal{T}$, including $\mathcal{E}$. To distinguish different (or potentially different) powers of $\mathcal{T}$, we write $\mathcal{T}^{\Phi_{1}}$ and $\mathcal{T}^{\Phi_{2}}$. Let $\mathcal{T}^{\Phi}[w]=\mathcal{T}^{\Phi} \otimes \mathcal{E}[w]$. 


\begin{tabular}{c|ccc} 
& $Y^{A}$ & $Z^{A c}$ & $X^{A}$ \\
\hline$Y_{A}$ & 0 & 0 & 1 \\
$Z_{A}{ }^{b}$ & 0 & $\mathbf{g}^{b c}$ & 0 \\
$X_{A}$ & 1 & 0 & 0
\end{tabular}

Figure 1. The tractor metric

Also let $\mathcal{T}, \mathcal{T}^{\Phi}$ and $\mathcal{T}^{\Phi}[w]$ denote the spaces of sections of these bundles. A choice of metric $g \in \mathbf{c}$ determines a splitting of $\mathcal{T}$, i.e. an isomorphism

$$
\mathcal{T} \stackrel{g}{\cong} \mathcal{E}[1] \oplus \mathcal{E}_{b}[1] \oplus \mathcal{E}[-1]
$$

We may write $T \stackrel{g}{=}\left(\sigma, \mu_{b}, \rho\right)$ to indicate that $T$ is an invariant section of $\mathcal{T}$, and $\left(\sigma, \mu_{b}, \rho\right)$ is its image under the splitting given by (9). In general, a conformally related metric $\widehat{g}$ determines a different splitting of $\mathcal{T}$. If $T \stackrel{g}{=}\left(\sigma, \mu_{b}, \rho\right)$, then

$$
T \stackrel{\widehat{g}}{=}\left(\sigma, \mu_{b}+\sigma \Upsilon_{b}, \rho-\boldsymbol{g}^{c d} \Upsilon_{c} \mu_{d}-\frac{1}{2} \sigma \boldsymbol{g}^{c d} \Upsilon_{c} \Upsilon_{d}\right)
$$

To facilitate our computations, we introduce three algebraic splitting operators,

$$
Y^{A} \in \mathcal{E}^{A}[-1], \quad Z^{A b} \in \mathcal{T}^{A b}[-1]:=\mathcal{T}^{A} \otimes \mathcal{E}^{b} \otimes \mathcal{E}[-1], \quad X^{A} \in \mathcal{E}^{A}[1],
$$

which administer the isomorphism (9) determined by the metric $g \in \mathbf{c}$. If $T^{A} \underline{\underline{g}}$ $\left(\sigma, \mu_{b}, \rho\right)$, then $T^{A}=\sigma Y^{A}+\mu_{b} Z^{A b}+\rho X^{A}$. Note that (10) determines the transformations of $Y^{A}, Z^{A b}$, and $X^{A}$ under conformal change of metric. These are easily computed and are given explicitly in [35].

There is a conformally invariant tractor metric $h$ on $\mathcal{T}$ which is preserved by $\nabla^{\mathcal{T}}$. We let $h^{\#}$ denote the co-metric associated to $h$ on the dual bundle to $\mathcal{T}$. In terms of the splitting operators, the tractor metric is given by Figure 1. In a symbolic tractor expression with tractor indices, one may eliminate all references to $h$ and $h^{\#}$ as follows. First, if one index of $h$ or $h^{\#}$ is contracted with an index of some other tractor, one may eliminate the reference to $h$ or $h^{\#}$ by raising or lowering the index of this other tractor. On the other hand, if $h$ or $h^{\#}$ has only free indices, then one may express $h$ and $h^{\#}$ in terms of the splitting operators $X$, $Y$, and $Z$. For example, $h_{A B}=Z_{A}{ }^{c} Z_{B c}+X_{A} Y_{B}+Y_{A} X_{B}$, as noted in [35].

The tractor connection is usefully encoded in the formulae for the tractor LeviCivita coupled derivatives of the splitting operators:

$$
\nabla_{a} Y^{A}=\mathrm{P}_{a b} Z^{A b}, \quad \nabla_{a} Z_{b}^{A}=-\mathrm{P}_{a b} X^{A}-\mathrm{g}_{a b} Y^{A}, \quad \nabla_{a} X^{A}=Z_{a}^{A} .
$$

The curvature $\Omega$ of the tractor connection is defined by

$$
\left(\nabla_{i} \nabla_{j}-\nabla_{j} \nabla_{i}\right) V^{A}=\Omega_{i j}{ }^{A}{ }_{B} V^{B}
$$

for $V^{A} \in \mathcal{T}^{A}$. A basic computation using (11) shows that

$$
\Omega_{i j}{ }_{B}{ }_{B}=C_{i j}{ }_{m}{ }_{m} Z_{k}^{A} Z_{B}{ }^{m}+2\left(\nabla_{[i} \mathrm{P}_{j]}{ }^{k}\right) Z^{A}{ }_{k} X_{B}-2\left(\nabla_{[i} \mathrm{P}_{j] m}\right) X^{A} Z_{B}{ }^{m} .
$$

We will also use the conformally invariant tractor $W$-curvature $W_{A B C E}$ as described in e.g. [35]:

$$
W_{A B C E}=(n-4) Z_{A}^{a} Z_{B}^{b} \Omega_{a b C E}-2 X_{[A} Z_{B]}^{b} \nabla^{p} \Omega_{p b C E} .
$$


From this formula and well known results it follows easily that $\mathbf{c}$ is conformally flat if and only if $W_{A B C E}=0$.

The notation $\psi:\left.\mathcal{T}^{\Phi_{1}}[w] \rightarrow \mathcal{T}^{\Phi_{2}}[w-c]\right|_{\Sigma}$ or $\psi: \mathcal{T}^{\Phi_{1}}[w] \rightarrow \mathcal{T}^{\Phi_{2}}[w-c]$ will indicate that $\psi$ is a family of natural differential operators parametrised by $w$ and $n$. Here $c$ is a real constant and $w$ is a real number. The family $\psi$ is a rule given by a universal symbolic formula. This rule defines an operator for all $(M, \mathbf{c})$ of dimension $n$, all bundles $\mathcal{T}^{\Phi_{1}}$, and all $w \in \mathbb{R}$. (We may require $(M, \mathbf{c})$ to meet various conditions, and $\mathcal{T}^{\Phi_{2}}$ will depend on $\mathcal{T}^{\Phi_{1}}$ and $\psi$.) If $w$ appears in the symbolic formula for $\psi$, we set $w$ equal to the weight of the bundle on which $\psi$ acts, unless we explicitly indicate otherwise. Since $\psi$ is given by a universal symbolic formula, we will sometimes refer to $\psi$ as an "operator" rather than a family of operators.

One important family of conformally invariant natural operators on weighted tractors is the family $D: \mathcal{T}^{\Phi}[w] \rightarrow \mathcal{T}^{A} \otimes \mathcal{T}^{\Phi}[w-1]$ defined as follows:

$$
D^{A} V=w(n+2 w-2) Y^{A} V+(n+2 w-2) Z^{A b} \nabla_{b} V-X^{A}(\Delta+w \mathrm{~J}) V .
$$

Cf. [3]. For developments of $D$ and proofs of its conformal invariance, see [21, 30]. Another important family of natural operators is the family $\square: \mathcal{T}^{\Phi}[w] \rightarrow \mathcal{T}^{\Phi}[w-2]$ given by

$$
\square V=(\Delta+w \mathrm{~J}) V .
$$

If $w=1-n / 2$, then $\square$ is the Yamabe operator, which is conformally invariant. We will use $D$ and $\square$ in our main operator constructions.

3.3. Conformal hypersurfaces. Let a hypersurface $\iota: \Sigma \rightarrow M$ be given. In this subsection, we present the necessary elements of basic conformal hypersurface geometry.

Let $g, \widehat{g} \in \mathbf{c}$ be given. These metrics induce conformally related metrics $\bar{g}$ and $\widehat{\bar{g}}$ on $\Sigma$, and so $\mathbf{c}$ induces a conformal structure $\overline{\mathbf{c}}$ on $\Sigma$. We term $\overline{\mathbf{c}}$ the intrinsic conformal structure of $\Sigma$. If $n \geq 4$, this conformal structure determines an intrinsic version of each of the constructions and results from Section 3.2 in the usual way. (We treat the $n=3$ case in Section 3.4, below.) Let $\bar{g}$ denote the intrinsic conformal metric, and let $\left(\overline{\mathcal{T}}, \bar{\nabla}^{\overline{\mathcal{T}}}\right)$ denote the intrinsic tractor bundle and its connection on $\Sigma$. (In fact, we shall usually write $\bar{\nabla}$ rather than $\bar{\nabla}^{\overline{\mathcal{T}}}$.) The conformally invariant (and $\bar{\nabla}$-parallel) metric on $\overline{\mathcal{T}}$, the intrinsic tractor metric, shall be denoted $\bar{h}$ and has signature $(n, 1)$. We identify $\left.\mathcal{E}[w]\right|_{\Sigma}$ with $\overline{\mathcal{E}}[w]$ in the obvious way.

Now let a local defining function, $t$, for $\Sigma$, as in Section 2, be given. Henceforth, we let

$$
n_{a}:=d t /|d t|_{g} .
$$

Thus $n_{a}$ is now a weight 1 conformally invariant conormal field for $\Sigma$ and, more generally, for the $\Sigma_{t}$ foliation. Let $\overline{\boldsymbol{g}}_{a b}:=\boldsymbol{g}_{a b}-n_{a} n_{b}$. This tensor extends the intrinsic conformal metric of $(\Sigma, \overline{\mathbf{c}})$ to a neighbourhood of $\Sigma$; its restriction gives the conformal metric on each leaf $\Sigma_{t_{0}}$. Given $g \in \mathbf{c}$, we again define $L_{a b}, H$, and $\stackrel{o}{L}_{a b}$ on a neighbourhood of $\Sigma$ as in Section 3.1, above. This time, however, we 
replace $\bar{g}$ with $\bar{g}$, and we use (17) to define $n_{a}$. As a consequence, $L_{a b}, H$, and $\stackrel{o}{L}_{a b}$ will have weights $1,-1$, and 1 , respectively. This convention will hold for the remainder of this paper. In fact, all formulae will now carry conformal weights, unless we note otherwise. This use of weights simplifies the transformation of the formulae under conformal rescaling. The formulae and results from Section 3.1 carry over to the present setting in the obvious way.

These remarks apply to natural differential operators, of course, and this leads to the following proposition.

Proposition 3.2. Let a pair $\left(w_{1}, w_{2}\right) \in \mathbb{R}^{2}$ and natural differential operators $P: \mathcal{T}^{\Phi}\left[-w_{1}\right] \rightarrow \mathcal{T}^{\Phi}\left[-w_{2}\right]$ and $P^{\prime}: \mathcal{T}^{\Phi} \rightarrow \mathcal{T}^{\Phi}$ be given. Finally, suppose that $P=\left(\xi^{g}\right)^{-w_{2}} P^{\prime}\left(\xi^{g}\right)^{w_{1}}$ for all $g \in \mathbf{c}$. Then $P^{\prime}$ is conformally covariant of bidegree $\left(w_{1}, w_{2}\right)$ if and only if $P$ is conformally invariant. A similar statement holds for operators mapping $\mathcal{T}^{\Phi}\left[-w_{1}\right]$ to $\left.\mathcal{T}^{\Phi}\left[-w_{2}\right]\right|_{\Sigma}$ and $\mathcal{T}^{\Phi}$ to $\left.\mathcal{T}^{\Phi}\right|_{\Sigma}$.

Proof. Note that $\left(\xi^{\widehat{g}}\right)^{w}=e^{-w \Upsilon}\left(\xi^{g}\right)^{w}$ for any $w \in \mathbb{R}$. The result thus follows from an elementary argument.

Remark 3.3. Proposition 3.2 allows us to identify conformally covariant and conformally invariant operators. In the main new operator constructions of this paper, we will work with conformally invariant operators, so for the rest of this paper, we will usually replace property (2) of Problem 1.2 with the following equivalent property: $P_{w, K}^{g}: \mathcal{E}[w] \rightarrow \overline{\mathcal{E}}[w-K]$ is conformally invariant. Here $K$ is as in Problem 1.2.

3.4. Tractors and conformal Gauss theory. We will subsequently exploit a conformally invariant replacement for the Gauss formula (77). Here we develop this machinery. In the following, we work along $\Sigma$, but the discussion applies to any leaf of the foliation without adjustment. Thus all quantities defined are extended into a neighbourhood of $\Sigma$. We assume $n \geq 3$, except as noted.

An elementary computation shows that

$$
\widehat{L}_{a b}=L_{a b}+\overline{\mathbf{g}}_{a b} \Upsilon^{c} n_{c},
$$

whence

$$
\widehat{H}=H+\Upsilon_{a} n^{a} .
$$

(Since $H$ and $n^{a}$ now carry weights, (19) differs from the conformal transformation law for $H$ that we discussed in Section 1, above.) It follows from (18) that $\stackrel{o}{L}_{a b}$ is conformally invariant. From (19) and (10) it follows that

$$
N^{A}=n_{b} Z^{A b}-H X^{A}
$$

is conformally invariant along $\Sigma$ and, more generally, along each leaf of the foliation $\Sigma_{t}$. This is the normal tractor as defined in [3]. It has conformal weight 0 . From $\mathrm{g}^{a b} n_{a} n_{b}=1$, it follows that $h^{A B} N_{A} N_{B}=1$. Let $\mathcal{T}_{\|}$be the (conformally invariant) subbundle of $\left.\mathcal{T}^{A}\right|_{\Sigma}$ whose fibre is the orthogonal complement (with respect to $h$ ) of $N^{A}$. If $n \geq 4$, then $\overline{\mathcal{T}}$ exists and has the same rank as $\mathcal{T}_{\mathbb{1}}$. This suggests the following proposition. This proposition follows [43, 57, which in turn follow an equivalent argument in [6]. 
Proposition 3.4. Suppose $n \geq 4$. Then along any leaf of the foliation, we may canonically and isometrically identify the bundles $\overline{\mathcal{T}}$ and $\mathcal{T}_{\|}$.

Proof. Let $g \in \mathbf{c}$ be given, and along any leaf of the foliation $\Sigma_{t}$, define an embed$\operatorname{ding} i: \overline{\mathcal{T}} \hookrightarrow \mathcal{T}$ as follows:

$$
\overline{\mathcal{T}} \ni T \stackrel{\bar{g}}{=}\left(\bar{\sigma}, \bar{\mu}_{b}, \bar{\rho}\right) \mapsto\left(\bar{\sigma}, \bar{\mu}_{b}+\bar{\sigma} H n_{b}, \bar{\rho}-\frac{1}{2} H^{2} \bar{\sigma}\right) \stackrel{g}{=} i T .
$$

The range of $i$ is clearly orthogonal to $N^{A}$. By (10), (19), and a basic calculation, it follows that $i: \overline{\mathcal{T}} \rightarrow \mathcal{T}_{\text {II }}$ is a conformally invariant bundle isomorphism compatible with the tractor metrics.

In the case $n=3$, we can still define the intrinsic tractor bundle $\overline{\mathcal{T}}$ in the usual way (i.e. as in higher dimensions), or we can equivalently identify $\overline{\mathcal{T}}=\mathcal{T}_{\|}$along $\Sigma$ as in [6]. In any case, we then define the intrinsic tractor connection $\bar{\nabla}$ and tractor $D$-operator, $\bar{D}$, on $\overline{\mathcal{T}}$ as in that reference.

For all $n \geq 3$, we shall henceforth identify $\overline{\mathcal{T}}$ and $\mathcal{T}_{\| 1}$. We can thus use the same abstract indices for the intrinsic and ambient tractor bundles; sections of $\overline{\mathcal{T}}$ are characterised by orthogonality to $N^{A}$. In an obvious way, these conventions are extended to the dual tractor bundle, tensor products, and so forth.

The normal tractor gives a conformally invariant tractor analogue of a Riemannian hypersurface conormal. Along any hypersurface $\Sigma$, the ambient tractor bundle $\mathcal{T}$ decomposes directly and orthogonally into $\overline{\mathcal{T}} \oplus \mathcal{N}$, where $\mathcal{N}$ is the tractor subbundle generated by the normal tractor field $N^{A}$. Note that $\Pi_{A}{ }^{B}:=\delta_{A}{ }^{B}-N_{A} N^{B}$, as a section of $\operatorname{End}(\mathcal{T})$, gives the projection $\mathcal{T} \rightarrow \overline{\mathcal{T}}$, and using the abstract index notation, we write $\Pi_{A}{ }^{B}: \mathcal{T}^{A} \rightarrow \overline{\mathcal{T}}^{B}$.

Although Proposition 3.4 is not surprising, there is a slight subtlety involved. Specifically, in the case $n \geq 4$, the proposition shows that the tractor splitting (9) determined by $\bar{g} \in \overline{\mathbf{c}}$ is not related to the splitting determined by $g \in \mathbf{c}$ in the most naïve way. (But it is, if, on a particular hypersurface, we work in a scale with $H \equiv 0$. Compare to [6, 29].)

By Proposition 3.4 and the above discussion, we have the following.

Proposition 3.5. $\bar{h}_{A B}=h_{A B}-N_{A} N_{B}$ and $\bar{h}^{A B}=h^{A B}-N^{A} N^{B}$ for all $n \geq 3$.

Now suppose $n \geq 4$. Let $\bar{Y}^{A}, \bar{Z}^{A b}$, and $\bar{X}^{A}$, in $\left.\mathcal{T}^{A}[-1]\right|_{\Sigma},\left.\mathcal{T}^{A b}[-1]\right|_{\Sigma}$, and $\left.\mathcal{T}^{A}[1]\right|_{\Sigma}$, respectively, denote the algebraic splitting operators associated to $\overline{\mathcal{T}}$ as determined by the induced metric $\bar{g}$. From (21), we see that

$$
\bar{Y}^{A}=Y^{A}+H n_{b} Z^{A b}-\frac{1}{2} H^{2} X^{A}, \quad \bar{Z}^{A b}=\Pi_{c}{ }^{b} Z^{A c}, \quad \bar{X}^{A}=X^{A} .
$$

Let a section $V^{A}$ of $\overline{\mathcal{T}}$ be given, and suppose that $V^{A} \stackrel{\bar{g}}{=}\left(\bar{\sigma}, \bar{\mu}_{b}, \bar{\rho}\right)$. Then

$$
V^{A}=\bar{\sigma} \bar{Y}^{A}+\bar{\mu}_{b} \bar{Z}^{A b}+\bar{\rho} \bar{X}^{A} .
$$

On the other hand, if $V^{A}=\sigma Y^{A}+\mu_{b} Z^{A b}+\rho X^{A} \in \mathcal{T}_{\text {II }}$, then

$$
V^{A}=\sigma \bar{Y}^{A}+\mu_{a} \Pi_{b}{ }^{a} \bar{Z}^{A b}+\left(\rho+\frac{1}{2} H^{2} \sigma\right) \bar{X}^{A} .
$$

Equation (22) describes the inverse of the isomorphism $\overline{\mathcal{T}} \rightarrow \mathcal{T}_{\text {॥l }}$ (as in [57]). 
Suppose again that $n \geq 4$, and define a projected ambient connection $\widetilde{\nabla}$ on $\overline{\mathcal{T}}$ as in [57] by letting

$$
\widetilde{\nabla}_{c} T^{A}=\Pi_{B}{ }^{A} \Pi_{c}{ }^{e} \nabla_{e} T^{B}
$$

for all sections $T^{A}$ of $\overline{\mathcal{T}}$. In [57], Stafford defines the tractor contorsion, which has the property that

$$
\bar{\nabla}_{c} T^{B}-\widetilde{\nabla}_{c} T^{B}=C_{c A}^{B} T^{A}
$$

for any section $T^{B}$ of $\overline{\mathcal{T}}$. By [57],

$$
C_{c A}{ }^{B}=X_{A} Z^{B b} \mathcal{F}_{b c}-X^{B} Z_{A}{ }^{a} \mathcal{F}_{a c},
$$

where $\mathcal{F}_{b c}:=\overline{\mathrm{P}}_{b c}-\Pi_{b}{ }^{i} \Pi_{c}{ }^{j} \mathrm{P}_{i j}-H \stackrel{o}{L}_{b c}-\frac{1}{2} \overline{\boldsymbol{g}}_{b c} H^{2}$ is the conformally invariant Fialkow tensor. Formulae (23) and (24) will be useful in Section 4 below.

Some of the examples in Section 8 , below, will refer to the tractor second fundamental form. The tractor second fundamental form is a conformally invariant tractor prolongation $L_{A B} \in \overline{\mathcal{T}}_{A B}[-1]$ of the second fundamental form $L_{a b}$. We use the definition given in [43].

Definition 3.6. In dimensions $n=\operatorname{dim}(M) \geq 4$, the tractor second fundamental form is given by

$$
L_{A B}=(n-3) \bar{Z}_{A}^{a} \prod_{a}^{d} \nabla_{d} N_{B}-\bar{X}_{A} \bar{\nabla}^{a} \Pi_{a}{ }^{d} \nabla_{d} N_{B} .
$$

along $\Sigma$.

The tractor second fundamental form is conformally invariant. To see this, begin by letting $w \in \mathbb{R}$ and $n \geq 4$ be given. Define an operator $E_{A B}{ }^{a C}: \overline{\mathcal{E}}_{a} \otimes \overline{\mathcal{T}}_{C}[w] \rightarrow$ $\overline{\mathcal{T}}_{A B}[w-1]$ as follows. For any $T_{a C} \in \overline{\mathcal{E}}_{a} \otimes \overline{\mathcal{T}}_{C}[w]$, let

$$
E_{A B}^{a C} T_{a C}=(n+w-3) \bar{Z}_{A}^{a} T_{a B}-\bar{X}_{A} \bar{\nabla}^{a} T_{a B} .
$$

In this definition, $\bar{\nabla}$ acts as the intrinsic Levi-Civita connection on $\overline{\mathcal{E}}_{a}[w]$ and as the intrinsic tractor connection on $\overline{\mathcal{T}}_{C}$. An easy adaptation of (8) shows that $E_{A B}{ }^{a C}$ is conformally invariant. A substitution then shows that $L_{A B}=E_{A B}{ }^{a C} \prod_{a}{ }^{d} \nabla_{d} N_{C}$. Thus $L_{A B}$ is conformally invariant.

Various versions of the following proposition appear in [37, 43, 57].

Proposition 3.7. In dimensions $n \geq 4$,

$$
\begin{aligned}
& L_{A B}=(n-3) \bar{Z}_{A}{ }^{a} \bar{Z}_{B}{ }^{b} \stackrel{o}{L}_{a b}-2 \frac{n-3}{n-2} \bar{X}_{(A} \bar{Z}_{B)}{ }^{a} \bar{\nabla}^{b} \stackrel{o}{L}_{a b} \\
& \quad+\bar{X}_{A} \bar{X}_{B}\left(o_{a b} \bar{P}^{a b}+\frac{1}{n-2} \bar{\nabla}^{a} \bar{\nabla}^{b}{ }^{o}{ }_{a b}\right) .
\end{aligned}
$$

Here $(A B)$ indicates symmetrisation over $A$ and $B$.

The following corollary summarises some of the important properties of the tractor second fundamental form:

Corollary 3.8. In dimensions $n \geq 4, L_{A B}$ is conformally invariant, symmetric, and trace free. 


\section{BASES OF Operators}

One of the keys to our operator constructions will be to work with bases of finite-dimensional vector spaces of natural hypersurface operators. In this section, we will construct such bases and derive some results relating to our work with these bases. The development of these bases will involve families of hypersurface operators $\psi$ which satisfy the following hypotheses:

Hypotheses 4.1. The family $\psi$ is a family of natural hypersurface differential operators given by a single universal symbolic formula. This formula is a polynomial in $n_{a}, R_{a b}{ }^{c} d, \mathbf{g}_{a b}, \mathbf{g}^{a b}$, the modified mean curvature $G$, and Levi-Civita connection $\nabla$. In this formula, $\nabla$ never explicitly hits $n_{a}$ or $G$. If $\mathbf{g}_{a b}$ or $\mathbf{g}^{a b}$ appears in the formula, then $a$ and $b$ are contracted with indices on $n_{a}, R_{a b}{ }^{c}$, or $\nabla_{a}$. For some $c \in \mathbb{R}$, some infinite set $\mathrm{D}$ of dimensions, all Riemannian manifolds $(M, g)$ of all dimensions $n \in \mathrm{D}$, all hypersurfaces $\Sigma$ in such manifolds, and all $w \in \mathbb{R}$, the symbolic formula for $\psi$ determines an operator $\psi: \mathcal{E}[w] \rightarrow \overline{\mathcal{E}}[w-c]$. In the polynomial formula for $\psi$, the coefficients are real functions of $w$ and $n$ which are polynomial in $w$ and rational in $n$.

The set D in Hypotheses 4.1 will be important in our work with the spaces $\mathcal{Q}_{K, c, w}$, $\mathcal{R}_{K, c, 0}$, and $\mathcal{S}_{K, c, 0}$, below. The trace-free part, $\stackrel{\circ}{L}_{a b}$, of the second fundamental form appears in many of the example formulae in Section 8 , below, but these appearances of $\stackrel{o}{L}_{a b}$ result from manipulation of other computed formulae.

In Hypotheses 4.1 and throughout this section, we may work with general Riemannian metrics. All of the results of this section continue to hold, however, if we assume that all metrics are conformally flat. In some situations, we will implicitly modify Hypotheses 4.1 by adding the assumption that all metrics are conformally flat. We do this, for example, in the proof of Theorem 5.12, below. Theorem 5.16, below, implicitly treats two cases, namely (1) conformally flat metrics and (2) general metrics, including conformally flat metrics. The proof of this theorem will implicitly require two separate applications of the theory of this section. Some of our other proofs will also implicitly treat the above two cases as separate cases.

Our development of operator bases and related results will require some groundwork. One key idea will be the idea of the mass of an operator, tensor, tractor, or combination of these. The concept of mass will allow us to construct finitedimensional vector spaces of operators and classes of operators. We will also use the concept of mass in Section 5.3, below, to estimate the order of a differential operator. We define mass as follows. Let $\mathcal{P}$ be a symbolic formula which is polynomial in $n_{a}, R_{a b}{ }^{c} d, \mathbf{g}_{a b}, \mathbf{g}^{a b}$, the coupled Levi-Civita connection $\nabla$, the splitting operators $Y^{A}, Z^{A b}$, and $X^{A}$, the tractor metric $h$, its co-metric $h^{\#}$, and real powers of $\xi^{g}$. Suppose that the coefficients in this polynomial are real rational functions of $n$ which may depend polynomially on a weight $w$. We will say that $R_{a b}{ }^{c}$ has mass $2, \nabla$ and $Y^{A}$ have mass $1, n^{a}, \mathbf{g}_{a b}, \mathbf{g}^{a b}, Z^{A b}, h, h^{\#}$, and powers of $\xi^{g}$ have mass 0 , and $X^{A}$ has mass -1 . All coefficients in $\mathcal{P}$ will have mass 0 . We define the mass of any given term of $\mathcal{P}$ to be the sum of the masses of the expressions that 
appear in the term. In this paper, all of the terms in a given symbolic formula $\mathcal{P}$ will always have the same mass, and we will say that this common mass is the mass of $\mathcal{P}$ (or the mass of the operator, tensor, or tractor corresponding to $\mathcal{P}$ ).

The use of (11), (12), and Figure 1 preserves mass. If one commutes covariant derivatives, one typically generates terms containing curvatures, but these terms have the same mass as the original expression. Recall that $L_{a b}=\Pi_{a}{ }^{c} \nabla_{c} n_{b}$. Thus $L_{a b}$ has mass 1 , and hence $H$ also has mass 1 . From (20) it then follows $N^{A}$ has mass 0. Similarly, $\bar{X}^{A}$ has mass $-1, \bar{Z}^{A b}$ has mass $0, \delta, D^{A}, \bar{\nabla}, \stackrel{o}{L}_{a b}, \bar{Y}^{A}, C_{c A}{ }^{B}$, and $L_{A B}$ have mass 1 , and $\delta_{2}, \Delta, \square, \bar{R}_{a b c d}, C_{a b c d}, \mathrm{P}_{a b}, \mathrm{~J}, \Omega_{i j}{ }_{B}{ }_{B}$, and $W_{A B C E}$ have mass 2. In Section 8 , below, the operators in figures 2 and 3 have masses 3 and 4 , respectively.

In the proofs of propositions 4.6 and 4.9, below, we will use a certain technical procedure which we now describe. Let a family of natural hypersurface operators $\psi$ be given, and suppose that $\psi$ satisfies Hypotheses 4.1. Let $w, c, n$, and $\mathrm{D}$ be as in Hypotheses 4.1, and let $w=0$. Suppose there is an infinite set $S \subseteq \mathrm{D}$ such that in all dimensions $n \in S, \psi: \mathcal{E}[0] \rightarrow \overline{\mathcal{E}}[-c]$ is the zero operator. Let $m \in \mathrm{D}$ be given, and also let the following be given and fixed: a Riemannian manifold $(M, g)$ of dimension $m$, a hypersurface $\Sigma$ of $M$, a point $p \in \Sigma$, and a smooth function $V$ on $M$. Then $\left(\xi^{g}\right)^{c}(\psi V)(p)$ is given by a symbolic formula. In this symbolic formula, we have set $n$ equal to $m$, but suppose we now replace $m$ with an arbitrary integer parameter $n$. (In the symbolic formula for $\left(\xi^{g}\right)^{c}(\psi V)(p)$, we still keep $(M, g), \Sigma, p$, and $V$ fixed.) The result is a real rational function $f$ of $n$. Of course $f(m)$ is equal to the original numerical value of $\left(\xi^{g}\right)^{c}(\psi V)(p)$. In the proofs of propositions 4.6 and 4.9, our plan will be to show that $f(n)=0$ for infinitely many $n \in \mathbb{Z}$. The key to doing this will be Proposition 4.2, below. The idea is to consider the action of a natural operator in a given dimension and relate this action to the action of the operator in other dimensions.

Proposition 4.2 will refer to the scale density $\xi^{g}$ associated to a Riemannian metric $g$ as defined in Section 3.2, above. Proposition 4.2 will also refer to the "natural" elevation of tensor indices. For $\nabla$ and the unit conormal field, this means that the index is down. The Riemannian curvature tensor naturally has one index up and three indices down. For $g$, it is natural to have both indices up or both down.

Proposition 4.2. Let a family of natural hypersurface operators $\psi$ be given, and suppose that $\psi$ satisfies Hypotheses 4.1. Let $c$, D, and $w$ be as in Hypotheses 4.1, and suppose that $w$ and $n$ do not appear in the universal symbolic formula for $\psi$. In this formula, suppose that all indices appear at their natural elevations, and consider $\left(\xi^{g}\right)^{c} \psi$ as acting on densities of weight zero only. For simplicity, we will write $\psi$ instead of $\left(\xi^{g}\right)^{c} \psi$. Let $m, m^{\prime} \in \mathrm{D}$ be given, and suppose that $m<m^{\prime}$. Finally, let a Riemannian manifold $(M, g)$ of dimension $m$, a hypersurface $\Sigma$ of $M$, and $V \in C^{\infty}(M)$ be given. Then there is a Riemannian manifold $\left(M^{\prime}, r\right)$ of dimension $m^{\prime}$, a hypersurface $\Sigma^{\prime}$ of $M^{\prime}$, and $V^{\prime} \in C^{\infty}\left(M^{\prime}\right)$ such that the following holds: For all $p \in \Sigma$, there is a $p^{\prime} \in \Sigma^{\prime}$ such that

$$
(\psi V)(p)=\left(\psi V^{\prime}\right)\left(p^{\prime}\right) \text {. }
$$


The proof of Proposition 4.2 appears in Appendix A, below.

We now turn to the definition of two general families of natural operators and one general family of operator classes. These families will lead to the bases that we mentioned above. Let $K \in \mathbb{Z}_{>0}, c, w \in \mathbb{R}$, and an infinite set $\mathrm{D} \subseteq \mathbb{Z}_{>0}$ be given. Let $\mathcal{Q}_{K, c, w}$ denote the set of all natural operators $\psi: \mathcal{E}[w] \rightarrow \overline{\mathcal{E}}[w-c]$ of mass $K$ defined in all dimensions $n \in \mathrm{D}$. We will assume that every $\psi \in \mathcal{Q}_{K, c, w}$ satisfies all of the properties in Hypotheses 4.1 with the following exception: The value of $w$ is always equal to the fixed value given above. For this choice of $w, \psi$ always maps $\mathcal{E}[w]$ to $\overline{\mathcal{E}}[w-c]$. We will also assume that the coefficients in the symbolic formula for $\psi$ are independent of $n$. These coefficients are thus real constants. We emphasise that every $\psi \in \mathcal{Q}_{K, c, w}$ is a rule which determines a hypersurface operator for every hypersurface $\Sigma$ in every Riemannian manifold $(M, g)$ of every dimension $n \in \mathrm{D}$. We never consider $\psi$ in dimensions $n \notin \mathrm{D}$. If $\psi \in \mathcal{Q}_{K, c, w}$ is zero, this means that $\psi$ is the zero operator in all dimensions $n \in \mathrm{D}$. It is clear that $\mathcal{Q}_{K, c, w}$ is a vector space over $\mathbb{R}$.

Proposition 4.3. Let $K \in \mathbb{Z}_{>0}$ and $c$, $w_{1}$, and $w_{2} \in \mathbb{R}$ be given, and define a mapping $\Phi: \mathcal{Q}_{K, c, w_{1}} \rightarrow \mathcal{Q}_{K, c, w_{2}}$ as follows. For all $\psi \in \mathcal{Q}_{K, c, w_{1}}$, let $\Phi(\psi): \mathcal{E}\left[w_{2}\right] \rightarrow$ $\overline{\mathcal{E}}\left[w_{2}-c\right]$ be the operator given by the universal symbolic formula for $\psi$. Then $\Phi$ is a vector space isomorphism.

Proof. Note that $\Phi$ is clearly linear and surjective. To show that $\Phi$ is injective, let $\psi \in \mathcal{Q}_{K, c, w_{1}}$ be given, and suppose that $\Phi(\psi)=0$. Also let $V \in \mathcal{E}\left[w_{1}\right]$ be given. Since powers of $\xi^{g}$ are parallel, it follows that

$$
\psi V=\left(\xi^{g}\right)^{w_{1}-w_{2}} \psi\left(\xi^{g}\right)^{w_{2}-w_{1}} V=\left(\xi^{g}\right)^{w_{1}-w_{2}} \Phi(\psi)\left(\xi^{g}\right)^{w_{2}-w_{1}} V=0 .
$$

Thus $\psi$ is the zero element of $\mathcal{Q}_{K, c, w_{1}}$, and $\Phi$ is injective.

Now let $K, c, w$, and $\mathrm{D}$ again be as above. Let $\mathcal{R}_{K, c, 0}$ denote the set of all natural differential operators $\psi: \mathcal{E}[0] \rightarrow \overline{\mathcal{E}}[-c]$ of mass $K$ which satisfy all of the properties of Hypotheses 4.1 except as follows. First, $w$ will always be zero. Thus every $\psi \in \mathcal{R}_{K, c, 0}$ will always map $\mathcal{E}[0]$ to $\overline{\mathcal{E}}[-c]$, and the coefficients in the universal symbolic polynomial formula for $\psi$ will be real rational functions of $n$. Also, in the universal symbolic formula for any given $\psi \in \mathcal{R}_{K, c, 0}$, we will allow the coefficients to be singular in some dimensions $n \in \mathrm{D}$. We will only consider $\psi$ in dimensions $n \in \mathrm{D}$. It may be helpful to think of $\mathcal{R}_{K, c, 0}$ as a set of symbolic operator formulae.

Let $F$ denote the field of all real rational functions of $n$. We would like to view $\mathcal{R}_{K, c, 0}$ as a vector space over $F$, but singularities in elements of $F$ make this difficult. To overcome this difficulty, we define a new space as follows. For each $\psi_{1}, \psi_{2} \in \mathcal{R}_{K, c, 0}$, we identify $\psi_{1}$ and $\psi_{2}$ if there is an $n_{0} \in \mathrm{D}$ such that in all dimensions $n \in \mathrm{D} \cap \mathbb{Z}_{\geq n_{0}}, \psi_{1}$ and $\psi_{2}$ are both regular and $\psi_{1}=\psi_{2}$. Let $\mathcal{S}_{K, c, 0}$ denote the set of equivalence classes that result from this identification. Then $\mathcal{S}_{K, c, 0}$ is clearly a vector space over $F$. For all $\psi \in \mathcal{R}_{K, c, 0}$, let $[\psi]$ denote the equivalence class of $\psi$ in $\mathcal{S}_{K, c, 0}$. For any $\psi \in \mathcal{R}_{K, c, 0}$, $[\psi]$ is the zero element of $\mathcal{S}_{K, c, 0}$ if and only if there is an $n_{0} \in \mathrm{D}$ such that $\psi$ is the zero operator in all dimensions $n \in \mathrm{D} \cap \mathbb{Z}_{\geq n_{0}}$. 
We will find bases for $\mathcal{Q}_{K, c, w}$ and $\mathcal{S}_{K, c, 0}$. We begin with $\mathcal{Q}_{K, c, w}$. Let $K, c$, and $w$ be given, as above. Let $S$ denote the set of all monomials $I_{g}$ in $n_{a}, R_{a b}{ }^{c}, \mathbf{g}_{a b}$, $\mathrm{g}^{a b}, G$, and $\nabla$ which have the following properties. First, each $I_{g} \in S$ has mass $K$ and coefficient 1. Each $I_{g}$ determines a natural operator $\psi: \mathcal{E}[w] \rightarrow \overline{\mathcal{E}}[w-c]$, and this operator $\psi$ satisfies Hypotheses 4.1 (but with the value of $w$ fixed). In each case, $I_{g}$ has the properties of the universal symbolic formula for $\psi$ discussed in Hypotheses 4.1. We include the subscript $g$ in the notation to indicate the metric used to define $n_{a}, R_{a b}^{c} d, G$, and $\nabla$ in the symbolic formula for $I_{g}$. This metric is a representative of the conformal class used in the definition of $\boldsymbol{g}_{a b}$ and $\boldsymbol{g}^{a b}$. One can easily verify that there are only finitely many ways of constructing a monomial $I_{g}$ of the above type; the key to doing so is the fact that $I_{g}$ must have mass $K$. It follows that $S$ is a finite set. Thus $S=\left\{I_{\alpha, g}\right\}_{\alpha \in A_{1}}$, where $A_{1}$ is a finite index set. The set $S$ clearly spans $\mathcal{Q}_{K, c, w}$.

Proposition 4.4. Let $\left\{I_{\alpha, g}\right\}_{\alpha \in A_{1}}$ be as above. Then there is a finite set $A \subseteq A_{1}$ such that $\left\{I_{\beta, g}\right\}_{\beta \in A}$ is a basis for $\mathcal{Q}_{K, c, w}$. The set $A$ and the symbolic formulae for the operators $I_{\alpha, g}$ are independent of $w$.

Proof. The claim that $A$ and the symbolic formulae are independent of $w$ follows from Proposition 4.3 .

Corollary 4.5. Let $\left\{I_{\beta, g}\right\}_{\beta \in A}$ be as above, and let $\psi \in \mathcal{R}_{K, c, 0}$ be given. Then $\psi=\sum_{\beta \in A} f_{\beta}(n) I_{\beta, g}$, where for each $\beta \in A, f_{\beta}(n)$ is some real rational function of $n$. We may assume that the functions $f_{\beta}(n)$ have the following property: If the coefficients of $\psi$ are regular in some dimension $n_{0} \in \mathrm{D}$, then each function $f_{\beta}(n)$ is regular for $n=n_{0}$.

Proof. Let $S=\left\{I_{\alpha, g}\right\}_{\alpha \in A_{1}}$ be as above, with $w=0$. By using the ideas that we discussed in our construction of this set, above, one can show that $\psi=$ $\sum_{\gamma \in A_{1}} h_{\gamma}(n) I_{\gamma, g}$. Here each coefficient $h_{\gamma}(n)$ is a real rational function of $n$. By Proposition 4.4, as applied to $\mathcal{Q}_{K, c, 0}$, there exist constants $C_{\gamma, \beta} \in \mathbb{R}$ such that for all $\gamma \in A_{1}, I_{\gamma, g}=\sum_{\beta \in A} C_{\gamma, \beta} I_{\beta, g}$. Thus $\psi=\sum_{\beta \in A}\left(\sum_{\gamma \in A_{1}} C_{\gamma, \beta} h_{\gamma}(n)\right) I_{\beta, g}$.

Proposition 4.6. Let $K \in \mathbb{Z}_{>0}$ and $c \in \mathbb{R}$ be given, and let notation be as above. Then $\left\{\left[I_{\beta, g}\right]\right\}_{\beta \in A}$ is a basis for $\mathcal{S}_{K, c, 0}$.

Proof. Let $\psi \in \mathcal{R}_{K, c, 0}$ be given. Then $\psi=\sum_{\beta \in A} f_{\beta}(n) I_{\beta, g}$, by Corollary 4.5, Here notation is as in the corollary. Thus $[\psi]=\sum_{\beta \in A} f_{\beta}(n)\left[I_{\beta, g}\right]$, and we see that $\left\{\left[I_{\beta, g}\right]\right\}_{\beta \in A}$ spans $\mathcal{S}_{K, c, 0}$.

We will use proof by contradiction to show that $\left\{\left[I_{\beta, g}\right]\right\}_{\beta \in A}$ is linearly independent. Suppose there is a set $\left\{f_{\beta}(n)\right\}_{\beta \in A} \subseteq F$ of real rational functions of $n$ which are not all zero such that

$$
\sum_{\beta \in A} f_{\beta}(n)\left[I_{\beta, g}\right]=0
$$

We may assume that all of the functions $f_{\beta}(n)$ are in fact polynomials in $n$. Let $\ell$ denote the largest degree of any of these polynomials. Let $A_{2}$ denote the set of all $\beta \in A$ such that $f_{\beta}(n)$ is nonzero, and for all $\beta \in A_{2}$, let $d_{\beta}$ and $b_{\beta}$ denote the degree and lead coefficient, respectively, of $f_{\beta}(n)$. Let $A_{3}=\left\{\beta \in A_{2} \mid d_{\beta}=\ell\right\}$. 
By (26) , there is an $n_{0} \in \mathrm{D}$ such that $\sum_{\beta \in A}\left(1 / n^{\ell}\right) f_{\beta}(n) I_{\beta, g}$ is the zero operator in all dimensions $n \in \mathrm{D} \cap \mathbb{Z}_{\geq n_{0}}$. Let a dimension $m \in \mathrm{D}$ be given. Also let a Riemannian manifold $(M, g)$ of dimension $m$, a hypersurface $\Sigma$ in $M$, a point $p \in \Sigma$, and $V \in C^{\infty}(M)$ be given. Next, let $n_{1}=\max \left\{n_{0}, m\right\}$. Finally, let $m^{\prime} \in \mathrm{D} \cap \mathbb{Z}_{>n_{1}}$ be given. Then by Proposition 4.2 , there is a Riemannian manifold $\left(M^{\prime}, r\right)$ of dimension $m^{\prime}$, a hypersurface $\Sigma^{\prime}$ of $M^{\prime}$, a function $V^{\prime} \in C^{\infty}\left(M^{\prime}\right)$, and a point $p^{\prime} \in \Sigma^{\prime}$ such that

$$
\left(\xi^{g}\right)^{c}\left(\sum_{\beta \in A_{2}} \frac{f_{\beta}\left(m^{\prime}\right)}{\left(m^{\prime}\right)^{\ell}} I_{\beta, g} V\right)(p)=\left(\xi^{r}\right)^{c}\left(\sum_{\beta \in A_{2}} \frac{f_{\beta}\left(m^{\prime}\right)}{\left(m^{\prime}\right)^{\ell}} I_{\beta, r} V^{\prime}\right)\left(p^{\prime}\right)=0 .
$$

Thus

$$
0=\lim _{n \rightarrow \infty}\left(\xi^{g}\right)^{c}\left(\sum_{\beta \in A_{2}} \frac{f_{\beta}(n)}{n^{\ell}} I_{\beta, g} V\right)(p)=\left(\xi^{g}\right)^{c}\left(\sum_{\beta \in A_{3}} b_{\beta} I_{\beta, g} V\right)(p) .
$$

Since $m \in \mathrm{D}$ was arbitrary, it follows that $\sum_{\beta \in A_{3}} b_{\beta} I_{\beta, g}$ is the zero operator for all $m \in \mathrm{D}$. This contradicts the linear independence of $\left\{I_{\beta, g}\right\}_{\beta \in A}$ in $\mathcal{Q}_{K, c, 0}$.

When we work with $\mathcal{Q}_{K, c, w}, \mathcal{R}_{K, c, 0}$, and $\mathcal{S}_{K, c, 0}$, operators will act on densities of a fixed weight. But many of the operators in this paper will act on densities of any weight. For some $c \in \mathbb{R}$ and every $w \in \mathbb{R}$, these operators will map $\mathcal{E}[w]$ to $\overline{\mathcal{E}}[w-c]$. To deal with such operators, we will need the following two propositions.

Proposition 4.7. Let $K \in \mathbb{Z}_{>0}, c \in \mathbb{R}$, and a family of natural hypersurface operators $\psi: \mathcal{E}[w] \rightarrow \overline{\mathcal{E}}[w-c]$ of mass $K$ be given, and suppose that $\psi$ satisfies Hypotheses 4.1. Suppose also that the universal symbolic formula for $\psi$ does not refer to $w$ or to $n$. Finally, let $\left\{I_{\beta, g}\right\}_{\beta \in A}$ be the basis for $\mathcal{Q}_{K, c, 0}$ described in Proposition 4.4. Then there exist real constants $C_{\beta}$ such that for all $w \in \mathbb{R}$, $\psi: \mathcal{E}[w] \rightarrow \overline{\mathcal{E}}[w-c]$ is given by the symbolic formula $\psi=\sum_{\beta \in A} C_{\beta} I_{\beta, g}$.

Proof. Let $w \in \mathbb{R}$ be given, and let $\Phi: \mathcal{Q}_{K, c, w} \rightarrow \mathcal{Q}_{K, c, 0}$ be as in Proposition 4.3. The universal symbolic formula for $\psi$ determines an element of $\mathcal{Q}_{K, c, w}$, and there are real constants $C_{\beta}$ such that $\Phi(\psi)=\sum_{\beta \in A} C_{\beta} I_{\beta, g}$. Since $\Phi$ is an isomorphism, $\psi: \mathcal{E}[w] \rightarrow \overline{\mathcal{E}}[w-c]$ satisfies $\psi=\sum_{\beta \in A} C_{\beta} I_{\beta, g}$. But the universal formula for $\psi$ does not refer to $w$, so the constants $C_{\beta}$ are independent of $w$. It follows that for all $w \in \mathbb{R}, \psi: \mathcal{E}[w] \rightarrow \overline{\mathcal{E}}[w-c]$ satisfies $\psi=\sum_{\beta \in A} C_{\beta} I_{\beta, g}$.

Proposition 4.8. Let $K \in \mathbb{Z}_{>0}$ and a family of natural operators $\psi$ be given. Suppose that this family satisfies Hypotheses 4.1 and that the operators $\psi$ have mass K. Let $c$ and $\mathrm{D}$ be as in Hypotheses 4.1. and let $\left\{I_{\beta, g}\right\}_{\beta \in A}$ be the basis for $\mathcal{Q}_{K, c, 0}$ described in Proposition 4.4. Then there exist real functions $f_{\beta}(w, n)$ such that for all $w \in \mathbb{R}$ and all $n \in \mathrm{D}, \psi: \mathcal{E}[w] \rightarrow \overline{\mathcal{E}}[w-c]$ is given by the symbolic formula $\psi=\sum_{\beta \in A} f_{\beta}(w, n) I_{\beta, g}$. For each $\beta \in A, f_{\beta}(w, n)$ is polynomial in $w$ and rational in $n$. If the parameter $w$ does not appear in the universal formula for $\psi$, then each function $f_{\beta}(w, n)$ is independent of $w$.

Proof. The result follows from Proposition 4.7 and an argument similar to the one we used in the proof of Corollary 4.5, above. 
We will use the following proposition in Section 7, below.

Proposition 4.9. Let $K \in \mathbb{Z}_{>0}$ and $c \in \mathbb{R}$ be given. Let $\mathrm{D}$ be as above, and let $\psi \in \mathcal{R}_{K, c, 0}$ be given. Suppose that the coefficient of every term in the universal symbolic formula for $\psi$ is regular for every $n \in \mathrm{D}$. Suppose also that for some $n_{1} \in \mathbb{Z}_{>0}, \psi$ is the zero operator in all dimensions $n \in \mathrm{D} \cap \mathbb{Z}_{>n_{1}}$. Then $\psi$ is the zero operator in all dimensions $n \in \mathrm{D}$.

Proof. By Corollary 4.5, we may may write $\psi=\sum_{\beta \in A} f_{\beta}(n) I_{\beta, g}$, where each coefficient $f_{\beta}(n)$ is a real rational function of $n$. Here $I_{\beta, g}$ and $A$ are as above. We may assume that $f_{\beta}(n)$ is regular at every $n \in \mathrm{D}$.

Let $m \in \mathrm{D}$ be given. Also let a Riemannian manifold $(M, g)$ of dimension $m$, a hypersurface $\Sigma$ in $M$, a point $p \in \Sigma$, and $V \in C^{\infty}(M)$ be given. Let $n_{2}=\max \left\{m, n_{1}\right\}$, and let $m^{\prime} \in \mathrm{D} \cap \mathbb{Z}_{>n_{2}}$ be given. Then by Proposition 4.2 , there is a Riemannian manifold $\left(M^{\prime}, r\right)$ of dimension $m^{\prime}$, a hypersurface $\Sigma^{\prime}$ of $M^{\prime}$, a function $V^{\prime} \in C^{\infty}\left(M^{\prime}\right)$, and a point $p^{\prime} \in \Sigma^{\prime}$ such that

$$
\left(\xi^{g}\right)^{c}\left(\sum_{\beta \in A} f_{\beta}\left(m^{\prime}\right) I_{\beta, g} V\right)(p)=\left(\xi^{r}\right)^{c}\left(\sum_{\beta \in A} f_{\beta}\left(m^{\prime}\right) I_{\beta, r} V^{\prime}\right)\left(p^{\prime}\right)=0 .
$$

Since $m^{\prime} \in \mathrm{D} \cap \mathbb{Z}_{>n_{2}}$ was arbitrary, $\left(\xi^{g}\right)^{c}\left(\sum_{\beta \in A} f_{\beta}\left(m^{\prime}\right) I_{\beta, g} V\right)(p)$ is a real rational function of $m^{\prime}$ which is zero for infinitely many distinct values of $m^{\prime}$. Thus $\left(\sum_{\beta \in A} f_{\beta}(m) I_{\beta, g} V\right)(p)=0$. Since $p$ and $V$ were arbitrary, it follows that $\psi$ is the zero operator in dimension $n=m$.

\section{INVARIANT OPERATOR CONSTRUCTIONS ALONG A HYPERSURFACE}

The basic example of a conformal boundary operator is the conformal Robin operator, as given by the formula (31). In this section, we develop the $\delta_{K}, \delta_{K}^{0}$ and $\delta_{J, k}$ operator families that we discussed in Section 1, above. Each gives, for most weights, higher-order analogues of $\delta$. The first key observation is that the conformal Robin operator is strongly conformally invariant, which means that by coupling formula (3) to the tractor connection, we obtain a conformally invariant operator along $\Sigma$ which acts on any weighted tractor bundle. We thus obtain

$$
\delta_{1}=\delta:\left.\mathcal{T}^{\Phi}[w] \rightarrow \mathcal{T}^{\Phi}[w-1]\right|_{\Sigma}
$$

along $\Sigma$ for any weight $w$. We use this to build the higher analogues of $\delta$. These new constructions will lead to solutions to problems 1.2 and 1.3 . The motivation for many steps of our procedure will center around the paramount Problem 1.3 . As we noted in Remark 3.3, above, we may identify conformally covariant and conformally invariant operators. This will be an important point in what follows.

5.1. Preliminary work. As above, we work locally along a hypersurface $\Sigma$ in a Riemannian conformal manifold $(M, \mathbf{c})$ with $\operatorname{dim}(M)=n \geq 3$. For convenience, the normal field, second fundamental form, and so on are extended off $\Sigma$ via a foliation, as discussed in sections 3.1 and 3.3, above. All quantities are thus defined on an open neighbourhood of $\Sigma$. We use that $\mathcal{E}$ is the same as the space 
of smooth sections of $\mathcal{E}[0]$. We begin by introducing a generalisation of the set $E$ of Problem 1.2.

Definition 5.1. Let $m \in \mathbb{Z}_{>0}, c \in \mathbb{R}$, and a family of natural hypersurface differential operators $\psi:\left.\mathcal{T}^{\Phi}[w] \rightarrow \mathcal{T}^{\Phi}[w-c]\right|_{\Sigma}$ of order at most $m$ be given. Let $E(\psi)$ denote the set of all weights $w$ such that $\psi$ fails to have transverse order $m$.

The set $E(\psi)$ of Definition 5.1 may depend on $n$. When we discuss $E(\psi)$ in this paper, the value of $m$ will always be clear from the context. If $m=0$, then $E(\psi)=\emptyset$. We will always find that $E(\psi)$ is independent of the bundle $\mathcal{T}^{\Phi}$.

The first main step in our operator constructions will be to define the $\delta_{K}$ operator family. We need some additional definitions and terminology that we introduce now.

Definition 5.2. Let $m \in \mathbb{Z}_{\geq 3}$, a set $\mathrm{D} \subseteq \mathbb{Z}_{\geq 3}$, and a family of natural differential operator $\psi$ be given, and suppose that $\psi$ is well-defined in all dimensions $n \in \mathrm{D}$. Also suppose that $\psi$ has order at most $m$ in all dimensions $n \in \mathrm{D}$ and for operands of all possible weights. Then lots and ltots will denote terms in a universal symbolic formula for $\psi$ which, in all dimensions $n \in \mathrm{D}$ and for operands of all possible weights, have order less than $m$ and transverse order less than $m$, respectively.

To simplify our discussions it will be convenient to introduce a notion that is very specific to the details of the formulae that we use. First observe that a symbolic formula for a natural differential operator may involve terms that move tractor indices in a way that we now describe. Let $T^{a b} \in \mathcal{E}^{a b}$ be given, and define an operator $\psi:\left.\mathcal{T}^{A}[w] \rightarrow \mathcal{T}^{A}[w]\right|_{\Sigma}$ by letting

$$
\psi V^{A}=T^{a b} \nabla_{a} \nabla_{b} V^{A}
$$

for all $V^{A} \in \mathcal{T}^{A}[w]$. Then

$$
\psi V^{A}=T^{a b} \nabla_{b} \nabla_{a} V^{A}+T^{a b} \Omega_{a b}{ }_{B}{ }_{B} V^{B},
$$

by (12). In one term of (29), the tractor index $A$ has moved off of $V$ and onto $\Omega$. We say that the operator formula given by the right-hand side of (29) moves tractor indices. The formula given by the right-hand side of (28) fixes tractor indices. One can extend these ideas to other operators and to tractor bundles of higher rank in the obvious way. We emphasise that the property of fixing or moving tractor indices is a property of an operator formula and not a property of an operator.

At many points in our operator constructions, we will work with a symbolic operator formula which satisfies the following hypotheses:

Hypotheses 5.3. The symbolic operator formula fixes tractor indices and is a polynomial in $n^{a}, R_{a b}{ }^{c}, \mathbf{g}_{a b}, \mathbf{g}^{a b}, H$, and the coupled Levi-Civita tractor connection $\nabla$. The coefficients of this polynomial are real functions of $w$ and $n$ which are polynomial in $w$ and rational in $n$. Within the symbolic operator formula, $\nabla$ never explicitly hits $n^{a}$ or $H$. For some $c \in \mathbb{R}$, the symbolic operator formula defines a family of natural differential operators $\psi:\left.\mathcal{T}^{\Phi}[w] \rightarrow \mathcal{T}^{\Phi}[w-c]\right|_{\Sigma}$. 
All of our operator constructions will begin with the operator family $\delta_{K}$ of the following lemma.

Lemma 5.4. For any $j \in \mathbb{Z}_{\geq 0}$, let $K=j+1$. Then the formula

$$
\delta_{j+1}:=N^{A_{1}} N^{A_{2}} \cdots N^{A_{j}} \delta D_{A_{1}} D_{A_{2}} \cdots D_{A_{j}}
$$

defines a family of natural hypersurface operators $\delta_{K}:\left.\mathcal{T}^{\Phi}[w] \rightarrow \mathcal{T}^{\Phi}[w-K]\right|_{\Sigma}$ of mass $K$. These operators are conformally invariant and may be given by a universal symbolic formula which satisfies Hypotheses 5.3 and is polynomial in $n$.

Proof. The claims of the lemma are clear by the formulae of (3), (11), (15), (20), (27), and Figure 1.

We will show that $\delta_{K}$ has order at most $K$, and we will find $E\left(\delta_{K}\right)$. To do this, we will need several results. For any $k \in \mathbb{Z}_{>0}$, let $\nabla_{n}^{k}$ denote $n^{a_{1}} \cdots n^{a_{k}} \nabla_{a_{1}} \cdots \nabla_{a_{k}}$, where $\nabla$ is the coupled Levi-Civita tractor connection. This operator has transverse order $k$.

Lemma 5.5. Let $s \in \mathbb{R}$ and $k \in \mathbb{Z}_{>0}$ be given. Then for any $V \in \mathcal{T}^{\Phi}[s]$,

$$
N^{A} \nabla_{n}^{k} D_{A} V=(n+2 s-2-k) \nabla_{n}^{k+1} V+k\left(\nabla_{n}^{k+1}-\nabla_{n}^{k-1} \Delta\right) V+\text { lots . }
$$

Here the lots can be given by a symbolic formula which satisfies Hypotheses 5.3 .

Proof. This is a trivial consequence of the formula (15) for the tractor $D$-operator. Verification in detail uses (11), (20), and the rules for the tractor metric given in Figure 1 .

We want to base an induction around this result. To do this, we must deal with terms similar to those in the second expression on the right-hand side of (31). The following is result is easily verified.

Lemma 5.6. Let nonnegative integers $p, q, r, s$, and $k$ be given, and suppose $p+2 q=r+2 s=k>0$. Then $\nabla_{n}^{p} \Delta^{q}-\nabla_{n}^{r} \Delta^{s}$, as an operator acting on densities or weighted tractors, is an operator of transverse order less than $k$.

Our induction will also require the following lemma.

Lemma 5.7. Let $K \in \mathbb{Z}_{>0}, c \in \mathbb{R}$, and a family of natural differential operators $O p:\left.\mathcal{T}^{\Phi}[w] \rightarrow \mathcal{T}^{\Phi}[w-c]\right|_{\Sigma}$ be given, and suppose that $O p$ can be given by a symbolic formula which satisfies Hypotheses 5.3. Suppose also that, for all $w \in \mathbb{R}$, $O p$ has order at most $K$. Then $N^{A} O p D_{A}$ can also be given by a symbolic formula which satisfies Hypotheses 5.3, and when acting on tractors of any weight $w \in \mathbb{R}$, this operator has order at most $K+1$. For any given $w \in \mathbb{R}$, if $O p: \mathcal{T}^{\Phi}[w] \rightarrow$ $\left.\mathcal{T}^{\Phi}[w-c]\right|_{\Sigma}$ has transverse order at most $K-1$, then for this value of $w$,

has transverse order at most $K$.

$$
N^{A} O p D_{A}:\left.\mathcal{T}^{\Phi}[w+1] \rightarrow \mathcal{T}^{\Phi}[w-c]\right|_{\Sigma}
$$

Proof. The result follows from (11), (15), (20), and Figure1. The key points are as follows. First, the leading part of $D_{A}$ is $-X_{A} \Delta$. We may assume that $O p$ is given by a symbolic formula which satisfies Hypotheses 5.3, so in the symbolic formula 
for $-N^{A} O p X_{A}$, we may assume that the tractor indices $A$ are in the locations shown. But $N^{A} X_{A}=0$, so in $-N^{A} O p X_{A}$, we may assume that at least one $\nabla$ hits $X_{A}$. It follows that $-N^{A} O p X_{A}$ is an operator of order at most $K+1$.

Now let $w \in \mathbb{R}$ be given, and suppose that $O p:\left.\mathcal{T}^{\Phi}[w] \rightarrow \mathcal{T}^{\Phi}[w-c]\right|_{\Sigma}$ has transverse order at most $K-1$. To complete the proof of the lemma, we will show that $N^{A} O p D_{A}:\left.\mathcal{T}^{\Phi}[w+1] \rightarrow \mathcal{T}^{\Phi}[w-c]\right|_{\Sigma}$ has transverse order at most $K$. To do this, we need only consider the leading part $-X_{A} \Delta$ of $D_{A}$. As above, we assume that the symbolic formula for $O p$ satisfies Hypotheses 5.3, so we may write

$$
O p=T^{i_{1} i_{2} \ldots i_{K}} \nabla_{i_{1}} \nabla_{i_{2}} \ldots \nabla_{i_{K}}+\text { lots },
$$

for some component functions $T^{i_{1} i_{2} \ldots i_{K}}$. Let a defining function $t$ for $\Sigma$ and a section $V$ of $\mathcal{T}^{\Phi}[w+1]$ be given, and consider

$$
N^{A} T^{i_{1} \ldots i_{K}} \nabla_{i_{1}} \ldots \nabla_{i_{K}}\left(-X_{A} \Delta t^{K+1} V\right) .
$$

Along $\Sigma$, this is a linear combination of terms of the form

$$
N^{A} T^{i_{1} \ldots i_{K}}\left(-Z_{A i_{a}}\right)\left(\nabla_{i_{1}} t\right) \cdots\left(\nabla_{i_{\hat{a}}} t\right) \cdots\left(\nabla_{i_{K}} t\right)\left(\nabla_{j} t\right)\left(\nabla^{j} t\right) V .
$$

Here $\hat{a}$ indicates omission of $\left(\nabla_{i_{a}} t\right)$. By (2),$\nabla_{i} t$ is $\left(\xi^{g}\right)^{-1} n_{i}$, up to multiplication by a nowhere zero function, so (33) is equal to

$$
-\left(\xi^{g}\right)^{-K} n_{i_{1}} \cdots n_{i_{K}} T^{i_{1} \ldots i_{K}}\left(\xi^{g}\right)^{-1} V,
$$

up to a nonzero scale. But (34), and hence also (32), vanish along $\Sigma$, since $O p$ : $\left.\mathcal{T}^{\Phi}[w] \rightarrow \mathcal{T}^{\Phi}[w-c]\right|_{\Sigma}$ has transverse order at most $K-1$

Proposition 5.8. Let $K \in \mathbb{Z}_{>0}$ be given. Then for every real number $w, \delta_{K}$ : $\left.\mathcal{T}^{\Phi}[w] \rightarrow \mathcal{T}^{\Phi}[w-K]\right|_{\Sigma}$ has order at most $K$, and along $\Sigma$,

$$
\delta_{K}=\left[\prod_{i=1}^{K-1}(n+2 w-K-i)\right] \nabla_{n}^{K}+\text { ltots . }
$$

Here the ltots can by given by a symbolic formula which satisfies Hypotheses 5.3. If $K=1$, we take the explicit product in (35) to be 1 .

Proof. This follows by induction and lemmas 5.5, 5.6, and 5.7.

Let $K \in \mathbb{Z}_{>0}$ be given. By Proposition 5.8 , it follows that $E\left(\delta_{K}\right)$ is the set of all $w$ that solve $\prod_{i=1}^{K-1}(n+2 w-K-i)=0$. Thus $E\left(\delta_{1}\right)=\emptyset$, and for any $K \in \mathbb{Z}_{\geq 2}$,

$$
E\left(\delta_{K}\right)=\left\{\frac{2 K-1-n}{2}, \frac{2 K-2-n}{2}, \cdots, \frac{K+1-n}{2}\right\} .
$$

For all $w \in \mathbb{R} \backslash E\left(\delta_{K}\right)$, the operator $\delta_{K}$ has transverse order $K$, and for all $w \in$ $E\left(\delta_{K}\right)$, it has transverse order less than $K$. Note that if $n=K+1$, then 0 is an exceptional weight. Our solutions to Problem 1.3 will therefore require some additional work, as we noted above. One of the keys to our work will be Proposition 5.10, below. In this proposition, $P_{2 k}: \mathcal{E}[k-n / 2] \rightarrow \mathcal{E}[-k-n / 2]$ is the GJMS operator of [41] of order $2 k$. To ensure that this operator exists, we assume that $n, k$, and $(M, \mathbf{c})$ satisfy the following condition: 
Condition 5.9. (1) $n$ is odd, or (2) $n$ is even and $k \leq n / 2$, or (3) (M, c) is conformally flat.

We will refer to Condition 5.9 at several points in our work.

Proposition 5.10. Let $k \in \mathbb{Z}_{>0}$ be given. There is a family of natural conformally invariant differential operators

$$
\mathcal{P}^{k}{ }_{A_{1} \cdots A_{k-1}}: \mathcal{E}[k-n / 2] \rightarrow \mathcal{T}_{A_{1} \cdots A_{k-1}}[-1-n / 2]
$$

such that for any $V \in \mathcal{E}[k-n / 2]$,

$$
(-1)^{k-1} X_{A_{1}} \cdots X_{A_{k-1}} P_{2 k} V=\square D_{A_{1}} \cdots D_{A_{k-1}} V+\mathcal{P}^{k}{ }_{A_{1} \cdots A_{k-1}} V .
$$

These operators are well-defined for all $n$ and $(M, \mathbf{c})$ which satisfy Condition 5.9. For the case in which $k \in\{1,2\}$ or $(M, \mathbf{c})$ is conformally flat, $\mathcal{P}^{k}{ }_{A_{1} \cdots A_{k-1}}=0$. In other cases, $\mathcal{P}^{k}{ }_{A_{1} \cdots A_{k-1}}=\Psi^{k}{ }_{A_{1} \cdots A_{k-1}}{ }^{P Q} D_{P} D_{Q}$, where $\Psi_{A_{1} \cdots A_{k-1}} P Q$ is given by a universal symbolic tractor formula. This formula is a polynomial in $X, D, W, h$, and $h^{\#}$ whose coefficients are real rational functions of $n$. Similarly, $\mathcal{P}^{k}{ }_{A_{1} \cdots A_{k-1}}$ is also given by such a tractor formula; every term of this formula is of degree at least 1 in $W$ and has mass $k+1$ and weight $-k-1$. In every term of the tractor formula for $\mathcal{P}^{k}{ }_{A_{1} \cdots A_{k-1}}$, at least one of the indices $A_{1}, \ldots, A_{k-1}$ appears on $a W$.

Proof. The proposition follows from a careful study of the statement and proof of Proposition 4.5 of [35]. Note that $W_{A B C E}$ vanishes if $(M, \mathbf{c})$ is conformally flat.

Remark 5.11. The proof of Proposition 5.10 uses the tractor formula for $P_{2 k}$ described in [35]. The construction of this tractor formula in general involves a finite number of choices; we assume that we have made and fixed these choices. As a result, the operators $\mathcal{P}^{k}{ }_{A_{1} \cdots A_{k-1}}$ and $\Psi^{k}{ }_{A_{1} \cdots A_{k-1}}{ }^{P Q}$ of Proposition [5.10, as well as all operators and curvatures derived from them, will always be uniquely determined.

5.2. Refining the boundary family in the conformally flat case. In the conformally flat case, it turns out that for every second weight in (36), $\delta_{K}$ is the zero operator. Dividing $\delta_{K}$ by the corresponding factors yields an improved family of operators. We state this precisely as follows.

Theorem 5.12. Let $K \in \mathbb{Z}_{>0}$ be given. There is a family of natural conformally invariant differential operators $\delta_{K}^{0}: \mathcal{E}[w] \rightarrow \overline{\mathcal{E}}[w-K]$ of order at most $K$ on conformally flat conformal manifolds $(M, \mathbf{c})$ of dimension $n \geq 3$ determined by the equation

$$
\left[\prod_{j=1}^{\left\lfloor\frac{K-1}{2}\right\rfloor}(n+2 w-2 K+2 j)\right] \delta_{K}^{0}=\delta_{K}
$$

and polynomial continuation in $w$. Here $\lfloor(K-1) / 2\rfloor$ denotes the integer part of $(K-1) / 2$. The $\delta_{K}^{0}$ family satisfies Hypotheses 4.1 with $\mathrm{D}=\mathbb{Z}_{\geq 3}$.

Proof. We may assume that $K \geq 3$. We will apply the results of Section 4 with $\mathrm{D}=\mathbb{Z}_{\geq 3}$. Let $\left\{I_{\beta, g}\right\}_{\beta \in A}$ be the basis for $\mathcal{Q}_{K, K, 0}$ described in Proposition 4.4. By Proposition 4.8, we may write $\delta_{K}=\sum_{\beta \in A} f_{\beta}(w, n) I_{\beta, g}$. Here each coefficient 
$f_{\beta}(w, n)$ is a polynomial in $w$ whose coefficients are real rational functions of $n$. By the polynomial division algorithm from elementary algebra, we may thus write

$$
f_{\beta}(w, n)=\left[\prod_{j=1}^{\left\lfloor\frac{K-1}{2}\right\rfloor}(n+2 w-2 K+2 j)\right] q_{\beta, n}(w)+r_{\beta, n}(w) .
$$

Here $q_{\beta, n}(w)$ and $r_{\beta, n}(w)$ are polynomials in $w$ whose coefficients are real rational functions of $n$. In performing the polynomial division, we are dividing by a polynomial with leading term $2^{b} w^{b}$, where $b=\lfloor(K-1) / 2\rfloor$. It follows that $q_{\beta, n}(w)$ and $r_{\beta, n}(w)$ are regular at all $n \in \mathrm{D}$, and $r_{\beta, n}(w)$ has degree less than $\lfloor(K-1) / 2\rfloor$.

Now let $j \in \mathbb{Z}$ and $V \in \mathcal{E}[K-j-n / 2]$ be given, and suppose that $1 \leq j \leq$ $\lfloor(K-1) / 2\rfloor$. An easy computation shows that $j<K-j$. By (30),

$$
\delta_{K} V=N^{A_{j}} N^{A_{j+1}} \cdots N^{A_{K-1}} \delta_{j} D_{A_{j}} D_{A_{j+1}} \cdots D_{A_{K-1}} V .
$$

By (15), (16), and Proposition 5.10 ,

$$
(-1)^{K-j} X_{A_{j}} X_{A_{j+1}} \cdots X_{A_{K-1}} P_{2(K-j)} V=D_{A_{j}} D_{A_{j+1}} \cdots D_{A_{K-1}} V .
$$

Next, note that $N^{A} X_{A}=0$, by (20) and Figure 1, Also note that $\delta_{j}$ has order at most $j$, by Proposition 5.8. Thus $\delta_{K} V=0$, by (39) and (40).

Now let $V_{0} \in \mathcal{E}[0]$ be given, and let $j$ be as above. Then

$$
\delta_{K}\left(\xi^{g}\right)^{K-j-n / 2} V_{0}=\sum_{\beta \in A} f_{\beta}(K-j-n / 2, n) I_{\beta, g}\left(\xi^{g}\right)^{K-j-n / 2} V_{0}=0
$$

along $\Sigma$. But $\nabla\left(\left(\xi^{g}\right)^{K-J-n / 2}\right)=0$, so $\sum_{\beta \in A} f_{\beta}(K-j-n / 2, n) I_{\beta, g} V_{0}=0$ along $\Sigma$. For all $\beta \in A, I_{\beta, g}$ determines an element $\left[I_{\beta, g}\right]$ of $\mathcal{S}_{K, K, 0}$. Since $V_{0}$ was arbitrary, our work shows that $\sum_{\beta \in A} f_{\beta}(K-j-n / 2, n)\left[I_{\beta, g}\right]$ is the zero element of $\mathcal{S}_{K, K, 0}$. Thus by Proposition 4.6, it follows that $f_{\beta}(K-j-n / 2, n)=0$ for all $\beta \in A$ and all $n \in \mathbb{Z}$. But then $r_{\beta, n}(K-j-n / 2)=0$ for all $\beta \in A$ and all $n \in \mathrm{D}$, by (38). Let $\beta \in A$ be given. Then for all $n \in \mathrm{D}, r_{\beta, n}(w)$ is a polynomial in $w$ having $\lfloor(K-1) / 2\rfloor$ distinct zeros. The coefficients of this polynomial are thus zero for all $n \in \mathrm{D}$ and hence for all $n \in \mathbb{Z}$. Thus $r_{\beta, n}(w)=0$ for all $w \in \mathbb{R}$ and all $n \in \mathbb{Z}$.

We now let $\delta_{K}^{0}=\sum_{\beta \in A} q_{\beta, n}(w) I_{\beta, g}$. A polynomial continuation argument shows that for all $w \in \mathbb{R}$, this operator is well-defined and conformally invariant.

Corollary 5.13. Let $E\left(\delta_{K}^{0}\right)$ be as in Definition 5.1. Then $E\left(\delta_{1}^{0}\right)=\emptyset$, and for all $K \in \mathbb{Z}_{\geq 2}$,

$$
E\left(\delta_{K}^{0}\right)=\left\{\frac{2 K-1-n}{2}, \frac{2 K-1-n}{2}-1, \cdots, \frac{2 K-1-n}{2}-\left\lfloor\frac{K-2}{2}\right\rfloor\right\} .
$$

For all $w \in \mathbb{R} \backslash E\left(\delta_{K}^{0}\right)$, the operator $\delta_{K}^{0}$ has transverse order $K$, and for all $w \in$ $E\left(\delta_{K}^{0}\right)$, it has transverse order less than $K$.

Proof. By Theorem 5.12 and Proposition 5.8, it follows that

$$
\left[\prod_{j=1}^{\left\lfloor\frac{K-1}{2}\right\rfloor}(n+2 w-2 K+2 j)\right] \delta_{K}^{0}=\left[\prod_{i=1}^{K-1}(n+2 w-K-i)\right] \nabla_{n}^{K}+\text { ltots }
$$


By Proposition 4.8, ltots $=\sum_{\beta \in A} h_{\beta}(w, n) I_{\beta, g}$. Here $A$ and $I_{\beta, g}$ are as above. Each coefficient $h_{\beta}(w, n)$ is a polynomial in $w$ whose coefficients are real rational functions of $n$. By reasoning as in the proof of Theorem [5.12, we find that

$$
\text { ltots }=\left[\prod_{j=1}^{\left\lfloor\frac{K-1}{2}\right\rfloor}(n+2 w-2 K+2 j)\right] \sum_{\beta \in A} s_{\beta, n}(w) I_{\beta, g},
$$

where $s_{\beta, n}(w)$ is polynomial in $w$ and rational in $n$. Polynomial continuation in $w$ shows that for all $w \in \mathbb{R}$, the operator $\sum_{\beta \in A} s_{\beta, n}(w) I_{\beta, g}$ has transverse order less than $K$. To obtain $\delta_{K}^{0}$, we divide (41) by the coefficient of $\delta_{K}^{0}$ on the left-hand side of (41). The corollary then follows.

We will use Corollary 5.13 to identify a critical operator in the proof of Theorem 7.10, below.

5.3. The refinement in the general case. We now consider arbitrary metrics and develop an additional refinement of $\delta_{K}: \mathcal{E}[w] \rightarrow \overline{\mathcal{E}}[w-K]$. The key to this new construction will be the operator family $P_{A_{1} \cdots A_{k}}$ of Proposition 5.14, below. In the definition of $\delta_{K}$ in (30), we will replace some of the $D$-operators with $P_{A_{1} \cdots A_{k}}$. Then, depending on parameters, we will divide the resulting operator by a polynomial in $w$ in much the same way as we did in our construction of $\delta_{K}^{0}$, above. Under certain conditions, this polynomial will be $(n+2 w-1-K)$. Suppose that we $d o$ in fact divide by $(n+2 w-1-K)$. Then for cases in which $n$ is even, $K=n-1$, and $w=0$, the refined operator will be a critical operator.

The key to the construction of $P_{A_{1} \cdots A_{k}}$ is the operator family $\mathcal{P}^{k}{ }_{A_{1} \cdots A_{k-1}}$ of Proposition 5.10. In that proposition, $\mathcal{P}^{k}{ }_{A_{1} \cdots A_{k-1}}$ acts on a section of $\mathcal{E}[k-n / 2]$. Any symbolic tractor formula for $\mathcal{P}^{k}{ }_{A_{1} \cdots A_{k-1}}$, however, determines a family of conformally invariant differential operators on $\mathcal{E}[w]$ for general weights $w \in \mathbb{R}$. In the next proposition, we will use this fact to construct $P_{A_{1} \cdots A_{k}}$.

Proposition 5.14. Let $k \in \mathbb{Z}_{>0}$ be given. By using the symbolic formula for $\mathcal{P}^{k} A_{2} \cdots A_{k}$, define a family of operators

$$
P_{A_{1} \cdots A_{k}}: \mathcal{E}[w] \rightarrow \mathcal{T}_{A_{1} \cdots A_{k}}[w-k]
$$

by letting

$$
P_{A_{1} \cdots A_{k}} V=D_{A_{1}} \ldots D_{A_{k}} V-X_{A_{1}} \mathcal{P}^{k}{ }_{A_{2} \cdots A_{k}} V
$$

for all $V \in \mathcal{E}[w]$. Then for any $V \in \mathcal{E}[k-n / 2]$,

$$
P_{A_{1} \cdots A_{k}} V=(-1)^{k} X_{A_{1}} \cdots X_{A_{k}} P_{2 k} V .
$$

The operators $P_{A_{1} \cdots A_{k}}$ are conformally invariant natural differential operators given by a universal symbolic formula which is a polynomial in $\mathbf{g}, Y, Z, X, h, h^{\#}, \nabla$, and the Riemannian curvature of $g$, where $g$ is a representative of the conformal structure on $M$. The coefficients of this polynomial are polynomial in $w$ and rational in $n$, and each term of the polynomial has mass $k$. The operators $P_{A_{1} \cdots A_{k}}$ are well-defined for all $n$ and $(M, \mathbf{c})$ which satisfy Condition 5.9. 
Proof. Let $V \in \mathcal{E}[k-n / 2]$ be given. By (15) and (37), it follows that

$$
(-1)^{k} X_{A_{1}} \cdots X_{A_{k}} P_{2 k} V=D_{A_{1}} \cdots D_{A_{k}} V-X_{A_{1}} \mathcal{P}^{k}{ }_{A_{2} \cdots A_{k}} V .
$$

Thus (43) holds. The other claims of the proposition follow from (13), (14), (15), and Proposition 5.10.

Our new refinement of $\delta_{K}$ will require additional groundwork. For any $E \subseteq \mathbb{R}$ and $x \in \mathbb{R}$, let $E+x=\{y+x \mid y \in E\}$.

Lemma 5.15. Let $J \in \mathbb{Z}_{>0}$, a real number $c$, and a family of natural differential operators $O p:\left.\mathcal{T}^{\Phi}[w] \rightarrow \mathcal{T}^{\Phi}[w-c]\right|_{\Sigma}$ be given. Suppose that Op has mass $J$ and order at most $J$ and can be given by a universal symbolic formula which satisfies Hypotheses 5.3. Suppose also that Op has transverse order less than J for all $w \in E(O p)$. Then there exist families of natural differential operators $O_{1}$ and $O_{2}$ having the following properties:

(1) For all $w \in \mathbb{R}, O_{1}$ and $O_{2} \operatorname{map} \mathcal{T}^{\Phi}[w]$ to $\left.\mathcal{T}^{\Phi}[w-c-1]\right|_{\Sigma}$.

(2) $N^{A} O p D_{A} V=(n+2 w-2-J) O_{1} V+O_{2} V$ for all sections $V \in \mathcal{T}^{\Phi}[w]$.

(3) $\mathrm{O}_{1}$ and $\mathrm{O}_{2}$ have mass $J+1$ and order at most $J+1$ and can be given by universal symbolic formulae which satisfy Hypotheses 5.3 .

(4) $O_{1}$ has transverse order less than $J+1$ for all $w \in E\left(O_{1}\right)$, and $E\left(O_{1}\right)=$ $E(O p)+1$.

(5) For all $w \in \mathbb{R}, \mathrm{O}_{2}$ has transverse order less than $J+1$.

Proof. Let $w \in \mathbb{R}$ be given. We will assume that $O p$ is given by a symbolic formula which satisfies Hypotheses [5.3. Thus $O p=T^{i_{1} \ldots i_{J}} \nabla_{i_{1}} \ldots \nabla_{i_{J}}+$ lots, where $T^{i_{1} \ldots i_{J}}$ are some component functions. By Lemma 5.7, $N^{A}$ lots $D_{A}$ is an operator of order less than $J+1$, which we include in $O_{2}$. Let $V \in \mathcal{T}^{\Phi}[w]$ and a defining function $t$ for $\Sigma$ be given, and consider the behaviour of $N^{A} T^{i_{1} \ldots i_{J}} \nabla_{i_{1}} \ldots \nabla_{i_{J}} D_{A} t^{J+1} V$ along $\Sigma$. We consider the various parts of $D_{A}$, beginning with $-X_{A} \Delta$. Along $\Sigma$, we have (44)

$$
\begin{aligned}
& N^{A} T^{i_{1} \ldots i_{J}} \nabla_{i_{1}} \ldots \nabla_{i_{J}}\left(-X_{A}\right) \Delta t^{J+1} V= \\
& \quad-\sum_{a=1}^{J}(J+1) ! N^{A} T^{i_{1} \ldots i_{J}} Z_{A i_{a}} \mathbf{g}^{k l}\left(\nabla_{i_{1}} t\right) \cdots\left(\nabla_{i_{\hat{a}}} t\right) \cdots\left(\nabla_{i_{J}} t\right)\left(\nabla_{k} t\right)\left(\nabla_{l} t\right) V= \\
& \quad-\sum_{a=1}^{J}(J+1) ! n_{i_{a}} T^{i_{1} \ldots i_{J}} \mathbf{g}^{k l}\left(\nabla_{i_{1}} t\right) \cdots\left(\nabla_{i_{\hat{a}}} t\right) \cdots\left(\nabla_{i_{J}} t\right)\left(\nabla_{k} t\right)\left(\nabla_{l} t\right) V .
\end{aligned}
$$

Here $\hat{a}$ denotes omission of the factor. By (17),

$$
n_{i_{a}} \mathbf{g}^{k l}\left(\nabla_{k} t\right) \nabla_{l} t=\frac{\nabla_{i_{a}} t}{|d t|_{g}} \mathbf{g}^{k l}\left(\nabla_{k} t\right) \nabla_{l} t=\left(\nabla_{i_{a}} t\right) \mathbf{g}^{k l}\left(\nabla_{k} t\right) n_{l}=\left(\nabla_{i_{a}} t\right) n^{k} \nabla_{k} t
$$

Thus by (44),

$$
\begin{aligned}
& N^{A} T^{i_{1} \ldots i_{J}} \nabla_{i_{1}} \ldots \nabla_{i_{J}}\left(-X_{A}\right) \Delta t^{J+1} V= \\
& \quad-J(J+1) ! T^{i_{1} \ldots i_{J}} n^{k}\left(\nabla_{i_{1}} t\right) \cdots\left(\nabla_{i_{J}} t\right)\left(\nabla_{k} t\right) V
\end{aligned}
$$

along $\Sigma$. 
Now consider the part of $D_{A}$ corresponding to $(n+2 w-2) Z_{A}^{k} \nabla_{k}$. Along $\Sigma$, we have

$$
\begin{aligned}
& N^{A} T^{i_{1} \ldots i_{J}} \nabla_{i_{1}} \ldots \nabla_{i_{J}}(n+2 w-2) Z_{A}{ }^{k} \nabla_{k} t^{J+1} V= \\
& \quad(n+2 w-2) T^{i_{1} \ldots i_{J}} n^{k} \nabla_{i_{1}} \ldots \nabla_{i_{J}} \nabla_{k} t^{J+1} V= \\
& \quad(n+2 w-2)(J+1) ! T^{i_{1} \ldots i_{J}} n^{k}\left(\nabla_{i_{1}} t\right) \cdots\left(\nabla_{i_{J}} t\right)\left(\nabla_{k} t\right) V .
\end{aligned}
$$

Thus

$$
\begin{aligned}
& N^{A} T^{i_{1} \ldots i_{J}} \nabla_{i_{1}} \ldots \nabla_{i_{J}} D_{A} t^{J+1} V= \\
& \quad(n+2 w-2-J)(J+1) ! T^{i_{1} \ldots i_{J}} n^{k}\left(\nabla_{i_{1}} t\right) \cdots\left(\nabla_{i_{J}} t\right)\left(\nabla_{k} t\right) V
\end{aligned}
$$

along $\Sigma$. Let $O_{1}:=T^{i_{1} \ldots i_{J}} n^{k} \nabla_{i_{1}} \ldots \nabla_{i_{J}} \nabla_{k}$. Then

$$
\begin{aligned}
& N^{A} T^{i_{1} \ldots i_{J}} \nabla_{i_{1}} \ldots \nabla_{i_{J}} D_{A}= \\
& \quad(n+2 w-2-J) O_{1}+N^{A} T^{i_{1} \ldots i_{J}} \nabla_{i_{1}} \ldots \nabla_{i_{J}} D_{A}-(n+2 w-2-J) O_{1} .
\end{aligned}
$$

The indicated sum of the second and third operators on the right-hand side of this equation is an operator of transverse order less than $J+1$, by our above work, and we include this operator in $\mathrm{O}_{2}$.

Let $w \in \mathbb{R} \backslash(E(O p)+1)$ and $p \in \Sigma$ be given, and choose a section $V$ of $\mathcal{T}^{\Phi}[w-1]$ such that $T^{i_{1} \ldots i_{J}} \nabla_{i_{1}} \ldots \nabla_{i_{J}} t^{J} V$ is nonzero at $p$. Then $T^{i_{1} \ldots i_{J}}\left(\nabla_{i_{1}} t\right) \cdots\left(\nabla_{i_{J}} t\right) V$ is nonzero at $p$. Thus $O_{1} t^{J+1} \xi^{g} V$ is nonzero at $p$, and hence $w \in \mathbb{R} \backslash E\left(O_{1}\right)$.

Finally, let $w \in E(O p)+1$ and $V \in \mathcal{T}^{\Phi}[w]$ be given. Along $\Sigma$, we have

$$
O_{1} t^{J+1} V=(J+1) n^{k}\left(\nabla_{k} t\right) \xi^{g} T^{i_{1} \ldots i_{J}} \nabla_{i_{1}} \ldots \nabla_{i_{J}} t^{J}\left(\xi^{g}\right)^{-1} V .
$$

But $w-1 \in E(O p)$, and $O p$ has transverse order less than $J$ for all weights in $E(O p)$. It follows that (45) vanishes along $\Sigma$. Thus $w \in E\left(O_{1}\right)$, and $O_{1}: \mathcal{T}^{\Phi}[w] \rightarrow$ $\left.\mathcal{T}^{\Phi}[w-c-1]\right|_{\Sigma}$ has transverse order less than $J+1$.

The following theorem gives our refinement of $\delta_{K}$ in the general case.

Theorem 5.16. Let $J, k \in \mathbb{Z}_{>0}$ be given. There is a family of natural conformally invariant differential operators $\delta_{J, k}: \mathcal{E}[w] \rightarrow \overline{\mathcal{E}}[w-k-J]$ determined as follows. For $k \leq J$,

$$
\delta_{J, k}=N^{A_{1}} \cdots N^{A_{k}} \delta_{J} P_{A_{1} \cdots A_{k}} .
$$

If $k>J$, then $\delta_{J, k}$ is determined by the equation

$$
(n+2 w-2 k) \delta_{J, k}=N^{A_{1}} \cdots N^{A_{k}} \delta_{J} P_{A_{1} \cdots A_{k}}
$$

and polynomial continuation in $w$. The $\delta_{J, k}$ family satisfies Hypotheses 4.1, and the operators $\delta_{J, k}$ have mass $J+k$ and order at most $J+k$ and are well-defined for all $n$ and $(M, \mathbf{c})$ which satisfy Condition 5.9. Moreover,

$$
E\left(\delta_{J, k}\right)=E\left(\delta_{J+k}\right) \backslash\{k-n / 2\},
$$

and $\delta_{J, k}$ has transverse order $J+k$ for all $w \in \mathbb{R} \backslash E\left(\delta_{J, k}\right)$ and transverse order less than $J+k$ for all $w \in E\left(\delta_{J, k}\right)$.

Proof. Suppose first that $k>J$ and suppose also that $V \in \mathcal{E}[k-n / 2]$. Then by Proposition 5.14,

$$
N^{A_{1}} \cdots N^{A_{k}} \delta_{J} P_{A_{1} \ldots A_{k}} V=N^{A_{1}} \cdots N^{A_{k}} \delta_{J}(-1)^{k} X_{A_{1}} \cdots X_{A_{k}} P_{2 k} V .
$$


This is zero along $\Sigma$, since $N^{A} X_{A}=0$. We will use this later in the proof.

Now consider general positive integers $J$ and $k$. Let $w \in \mathbb{R}$ be given, and suppose that $V \in \mathcal{E}[w]$. By the definition of $P_{A_{1} \ldots A_{k}}$,

$$
\begin{aligned}
& N^{A_{1}} \cdots N^{A_{k}} \delta_{J} P_{A_{1} \ldots A_{k}} V= \\
& \quad N^{A_{1}} \cdots N^{A_{k}} \delta_{J} D_{A_{1}} \ldots D_{A_{k}} V+N^{A_{1}} \cdots N^{A_{k}} \delta_{J}\left(-X_{A_{1}} \mathcal{P}^{k}{ }_{A_{2} \cdots A_{k}} V\right) .
\end{aligned}
$$

If $\mathcal{P}^{k}{ }_{A_{2} \cdots A_{k}}$ is nonzero, then every term of $\mathcal{P}^{k}{ }_{A_{2} \cdots A_{k}} V$ contains at least one occurrence of the tractor curvature $W$. We may use (13), (14), and (15) to expand all occurrences of $W$ and the tractor $D$-operator in the second term on the right-hand side of (47). We may then use the rules in Figure 1 to eliminate all occurrences of $X, Y$, and $Z$ in the resulting expression. By (13) and (14), each of the resulting terms will contain at least one occurrence of the Weyl tensor or the Schouten tensor. Since each term of (47) has mass $J+k$, it follows that the second term on the right-hand side of (47) has order less than $J+k$ for all $w \in \mathbb{R}$. This second term satisfies the conditions in Hypotheses 4.1.

By lemmas 5.7 and 5.15.

$$
N^{A_{1}} \cdots N^{A_{k}} \delta_{J} D_{A_{1}} \ldots D_{A_{k}} V=\left[\prod_{i=1}^{k}(n+2 w-2 k+i-1-J)\right] O_{1} V+O_{2} V
$$

for some natural operators $O_{1}$ and $O_{2}$ of mass $J+k$ and order at most $J+$ $k$. The operators $O_{1}$ and $O_{2}$ may be given by symbolic formulae which satisfy Hypotheses 5.3. The operator $O_{1}$ has transverse order less than $J+k$ for all $w \in E\left(O_{1}\right)$, and $E\left(O_{1}\right)=E\left(\delta_{J}\right)+k$. The operator $O_{2}$ has transverse order less than $J+k$ for all $w \in \mathbb{R}$. By (47) and (48),

$$
\begin{aligned}
& N^{A_{1}} \cdots N^{A_{k}} \delta_{J} P_{A_{1} \cdots A_{k}} V= \\
& \quad\left[\prod_{i=1}^{k}(n+2 w-2 k+i-1-J)\right] O_{1} V+O_{2} V+\text { lots } .
\end{aligned}
$$

Suppose now that $k \leq J$, and let $S$ denote the set of all $w$ for which the explicit product in (49) is zero. Then $E\left(\delta_{J, k}\right)=S \cup\left(E\left(\delta_{J}\right)+k\right)$. By (36) and an algebraic exercise, it follows that $E\left(\delta_{J, k}\right)=E\left(\delta_{J+k}\right)$. Since $k \leq J$, it follows that $k-n / 2 \notin E\left(\delta_{J+k}\right)$. Thus (46) holds in the case $k \leq J$.

Now suppose instead that $k>J$. Let $\mathrm{O}_{2} V+$ lots be as in (49). Then $\mathrm{O}_{2} V+$ lots $=$ $\mathrm{O}_{3} V$ for some natural operator $O_{3}$ of mass $J+k$. We apply Proposition 4.8 with $K=c=J+k$. We conclude that $O_{3}=\sum_{\beta \in A} f_{\beta}(w, n) I_{\beta, g}$, where $A$ and $I_{\beta, g}$ are as in Proposition 4.8. Here $f_{\beta}(w, n)$ is a polynomial in $w$ whose coefficients are real rational functions of $n$. By the polynomial division algorithm from elementary algebra, we may write

$$
f_{\beta}(w, n)=(n+2 w-2 k) q_{\beta, n}(w)+r_{\beta, n}
$$

for each $\beta \in A$. Here $q_{\beta, n}(w)$ is a polynomial in $w$ whose coefficients are real rational functions of $n$, and $r_{\beta, n}$ is a real rational function of $n$. We are dividing by a polynomial whose leading term is $2 w$. Thus if $f_{\beta}(w, n)$ is regular at some value of $n$, then $q_{\beta, n}(w)$ and $r_{\beta, n}$ are also regular for this value of $n$. 
We now work with the same operator formula, but we trivialise the density bundles as in Section 4, So we operate now on $V_{0} \in \mathcal{E}[0]$. Then

$$
O_{3}\left(\xi^{g}\right)^{k-n / 2} V_{0}=\sum_{\beta \in A} f_{\beta}(k-n / 2, n) I_{\beta, g}\left(\xi^{g}\right)^{k-n / 2} V_{0}=0
$$

along $\Sigma$, by (49) and our work at the beginning of this proof. Since $\nabla\left(\left(\xi^{g}\right)^{k-n / 2}\right)=$ 0 , it follows that $\sum_{\beta \in A} f_{\beta}(k-n / 2, n) I_{\beta, g} V_{0}=0$ along $\Sigma$. For each $\beta \in A, I_{\beta, g}$ determines an element $\left[I_{\beta, g}\right]$ of $\mathcal{S}_{J+k, J+k, 0}$. Since $V_{0} \in \mathcal{E}[0]$ was arbitrary, it follows that $\sum_{\beta \in A} f_{\beta}(k-n / 2, n)\left[I_{\beta, g}\right]$ is the zero element of $\mathcal{S}_{J+k, J+k, 0}$. Thus by Proposition 4.6, we see that $f_{\beta}(k-n / 2, n)=r_{\beta, n}=0$ for all $\beta \in A$ and all $n \in \mathbb{Z}$. We may therefore conclude that

$$
O_{3}=(n+2 w-2 k) \sum_{\beta \in A} q_{\beta, n}(w) I_{\beta, g}
$$

for general weights $w$. Let $O_{4}=\sum_{\beta \in A} q_{\beta, n}(w) I_{\beta, g}$. We may then let

$$
\delta_{J, k}:=\left[\prod_{i=1, i \neq J+1}^{k}(n+2 w-2 k+i-1-J)\right] O_{1}+O_{4} .
$$

Polynomial continuation in $w$ shows that if $w=k-n / 2$, then $O_{4}$ has transverse order less than $J+k$. Thus $O_{4}$ has transverse order less than $J+k$ for all $w \in \mathbb{R}$. This establishes (46). Polynomial continuation also shows that $\delta_{J, k}$ is well-defined and conformally invariant.

A simple key case is as follows.

Corollary 5.17. Let $n_{0} \in \mathbb{Z}_{\geq 4}$ and $n \in \mathbb{Z}_{\geq 3}$ be given. Also let $J=\left\lfloor\left(n_{0}-2\right) / 2\right\rfloor$, and let $k=\left\lfloor\left(n_{0}+1\right) / 2\right\rfloor$. Suppose that $k, n$, and $\mathbf{c}$ satisfy Condition [5.9. Then for all $w \in \mathbb{R}, \delta_{J, k}: \mathcal{E}[w] \rightarrow \overline{\mathcal{E}}\left[w-n_{0}+1\right]$ is well-defined and has order at most $n_{0}-1$.

Now suppose in addition that $n=n_{0}$. If the dimension $n$ is odd, then $E\left(\delta_{J, k}\right)=$ $\{0\} \cup\{i / 2 \mid 2 \leq i \leq n-3, i \in \mathbb{Z}\}$. On the other hand, if $n$ is even, then $E\left(\delta_{J, k}\right)=$ $\{i / 2 \mid 1 \leq i \leq n-3, i \in \mathbb{Z}\}$.

We will use Corollary 5.17 to identify a critical operator in the proof of Theorem 7.10, below.

\section{Conformal Dirichlet-to-Neumann Operators}

In this section, we construct conformally invariant Dirichlet-to-Neumann operators on a Riemannian conformal manifold with boundary $(M, \Sigma, \mathbf{c})$. Our construction uses the GJMS operators $P_{2 k}$ on $(M, \mathbf{c})$ and the hypersurface operator families of this paper. We begin with some groundwork in Section 6.1. We use standard theory of elliptic boundary problems as in [44, 46]; a summary of the key results needed from those sources is given in [6, Section 6]. 
6.1. Formally self-adjoint boundary problems. For any $K \in \mathbb{Z}_{>0}$, we will use $\delta_{K}^{T}$ to denote any of the operators of order and transverse order $K$ from Lemma 5.4 and theorems 5.12 and 5.16, above, as available according to context. For any $w \in \mathbb{R}$, let $\delta_{0}^{T}: \mathcal{E}[w] \rightarrow \overline{\mathcal{E}}[w]$ be given by $\delta_{0}^{T} u=\left.u\right|_{\Sigma}$. We also let $\delta_{0}$ denote $\delta_{0}^{T}$. The following lemma describes some cases in which the operators $\delta_{K}^{T}$ are available.

Lemma 6.1. Let a positive integer $k$ be given, and suppose $n, k$, and $(M, \mathbf{c})$ satisfy Condition 5.9. Let $i \in \mathbb{Z}$ be given, and suppose that $0 \leq i \leq 2 k-1$. Then there is an operator $\delta_{i}^{T}: \mathcal{E}[k-n / 2] \rightarrow \overline{\mathcal{E}}[k-n / 2-i]$ of order and transverse order $i$. In particular, $k-n / 2 \notin E\left(\delta_{i}^{T}\right)$.

Proof. If $i=0$, let $\delta_{i}^{T}=\delta_{0}$. Now suppose instead that $1 \leq i \leq k$. Then $k-n / 2 \notin$ $E\left(\delta_{i}\right)$, by (36)), so we may let $\delta_{i}^{T}=\delta_{i}$. Finally, suppose that $k+1 \leq i \leq 2 k-1$. Let $j=i-k$. Then $k-n / 2 \notin E\left(\delta_{j, k}\right)$, by Theorem [5.16, so we may let $\delta_{i}^{T}=\delta_{j, k}$.

Let $M$ be a compact conformal $n$-manifold with smooth boundary $\Sigma=\partial M$. Recall that a boundary problem $(A, B)$ is formally self-adjoint (FSA), or symmetric, if and only if

$$
\int_{M}[(A u) v-u A v]=0
$$

whenever $B u=B v=0$ on $\Sigma$.

Recall that each GJMS operator $P_{2 k}: \mathcal{E}[k-n / 2] \rightarrow \mathcal{E}[-k-n / 2]$ is conformally invariant and FSA [27, 42]. For each such GJMS operator, we will consider the associated conformal generalised Dirichlet problem $\left(P_{2 k}, B\right)$, where $B$ is the multiboundary operator

$$
B=\left(\delta_{0}^{T}, \delta_{1}^{T}, \cdots, \delta_{k-1}^{T}\right) .
$$

We will need the following observation.

Lemma 6.2. Let $k \in \mathbb{Z}_{>0}$ and a section $u$ of $\mathcal{E}[k-n / 2]$ be given. Suppose that $u$ is in the kernel of $B$, i.e.

$$
0=\delta_{0}^{T} u=\delta_{1}^{T} u=\cdots=\delta_{k-1}^{T} u \quad \text { along } \quad \Sigma .
$$

Then the $(k-1)$-jet of $u$ is zero at every point of $\Sigma$.

Proof. Let $s$ be any local defining function for $\Sigma$. We will use induction to show that for all $\ell \in\{1,2, \ldots, k\}$, there is a smooth section $u_{\ell}$ of $\mathcal{E}[k-n / 2]$ such that $u=s^{\ell} u_{\ell}$. Let $c \in\{2,3 \ldots, k\}$ be given, and suppose that $u=s^{c-1} u_{c-1}$ for some $u_{c-1} \in \mathcal{E}[k-n / 2]$. Then along $\Sigma, \delta_{c-1}^{T} u=F^{i_{1} \cdots i_{c-1}}\left(\nabla_{i_{1}} s\right) \cdots\left(\nabla_{i_{c-1}} s\right) u_{c-1}=0$ for some component functions $F^{i_{1} \cdots i_{c-1}}$. Since $\delta_{c-1}^{T}$ has transverse order $c-1$ in this context, it follows that $u_{c-1}=s u_{c}$ for some section $u_{c}$ of $\mathcal{E}[k-n / 2]$.

This leads to the following result.

Theorem 6.3. Let $k \in \mathbb{Z}_{>0}$ be given, and let $B$ be as in (50). Suppose that $n, k$, and $(M, \mathbf{c})$ satisfy Condition 5.9. Then the conformal generalised Dirichlet problem $\left(P_{2 k}, B\right)$ is FSA. 
Proof. Let sections $u$ and $v$ of $\mathcal{E}[k-n / 2]$ be given, and fix a metric $g \in \mathbf{c}$ for the purpose of calculating. Since $P_{2 k}$ is FSA, we have

$$
\int_{M}\left[\left(P_{2 k} u\right) v-u P_{2 k} v\right]=\int_{\Sigma} \operatorname{sk}_{P_{2 k}}(u, v),
$$

where $\operatorname{sk}_{P_{2 k}}(u, v)$ is a skew bilinear form on the Cauchy data and its tangential derivatives at the boundary $\Sigma$. To see this, repeatedly integrate terms of the left-hand side of (51) by parts in the usual way. All integrals over $M$ eventually cancel out, and the boundary integral of a bilinear form $b(u, v)$ remains. But this boundary integral is skew-symmetric in $u$ and $v$, so in this integral, we may replace $b(u, v)$ with its skew part. In each term of $\operatorname{sk}_{P_{2 k}}(u, v)$, either $u$ or $v$ is differentiated at most $k-1$ times, so the result follows from Lemma 6.2.

To construct our conformal Dirichlet-to-Neumann operators, we will work with well-posed boundary problems, in particular, boundary problems which satisfy the so-called Lopatinski-Shapiro condition (see e.g. [1, 6, 46]). We will assume that $M$ is compact with nonempty boundary $\Sigma$. We define the Lopatinski-Shapiro condition below. We will use the definition given in [6]. Our definition begins with the idea of elliptic and properly elliptic operators. Let a differential operator $A$ of order $j$ acting on $\mathcal{E}[w]$ be given, and let $a_{j}(x, \xi)$ denote the leading symbol of $A$. We say that $A$ is elliptic if there is a constant $C>0$ such that $\left|a_{j}(x, \xi)\right| \geq C|\xi|^{j}$ on $M$. Here $|\xi|^{2}=g^{a b} \xi_{a} \xi_{b}$. In this context, we will always assume that $j$ is even.

Now consider arbitrary $x \in \Sigma$ and $\eta_{a} \in \mathcal{E}_{a}$, as well as the polynomial $p(\tau)=$ $a_{j}\left(x, \eta_{a}+\tau n_{a}\right)$. We say that $A$ is properly elliptic if for all $x \in \Sigma$ and all $\eta_{a}$ not parallel to $n_{a}$ at $x$, the zeros of $p(\tau)$ are separated by the real axis. This means that one may label the zeros as $\tau_{i}^{ \pm}$, for $i=1, \ldots, j / 2$, and do this in such a way that $\operatorname{Im} \tau_{i}^{+}>0$ and $\operatorname{Im} \tau_{i}^{-}<0$ for all $i$.

We may now define the Lopatinski-Shapiro condition. Let $k \in \mathbb{Z}_{>0}$, a properly elliptic differential operator $A$ of order $2 k$, and a multi-boundary operator $O=$ $\left(O_{1}, \ldots, O_{k}\right)$ be given. For each $i$, suppose that $O_{i}$ has order $m_{i}$ and leading symbol $b_{i}(x, \xi)$. Suppose also that $0 \leq m_{1}<m_{2}<\cdots<m_{k}<2 k$. For every $i \in\{1, \ldots, k\}$, let $p_{i}(\tau)=b_{i}\left(x, \eta_{a}+\tau n_{a}\right)$. Let $\tau_{i}^{+}$be as above, and let $M^{+}=$ $\Pi_{i=1}^{k}\left(\tau-\tau_{i}^{+}\right)$. Let $I$ denote the principal ideal in $\mathbb{C}[\tau]$ generated by $M^{+}$. Each $f(\tau)$ in $\mathbb{C}[\tau]$ determines an element $[f(\tau)]$ of $\mathbb{C}[\tau] / I$. We say that $(A, O)$ satisfies the Lopatinski-Shapiro condition if, for all $x \in \Sigma$ and all $\eta_{a}$ not parallel to $n_{a}$ at $x$, the elements $\left[p_{i}(\tau)\right]$ are linearly independent in $\mathbb{C}[\tau] / I$.

If $n, k$, and $\mathbf{c}$ satisfy Condition [5.9, then $P_{2 k}$ is elliptic and properly elliptic. If $A=P_{2 k}$, then there is an $\alpha \in \mathbb{C}$ such that $M^{+}=(\tau-\alpha)^{k}$. We will use this formula for $M^{+}$in the proof of the following lemma.

Lemma 6.4. Let $k \in \mathbb{Z}_{>0}$ be given, and let $O$ denote the multi-boundary operator $\left(\delta_{0}, n^{a} \nabla_{a},\left(n^{a} \nabla_{a}\right)^{2}, \ldots,\left(n^{a} \nabla_{a}\right)^{k-1}\right)$. If $n, k$, and $(M, \mathbf{c})$ satisfy Condition 5.9, then the boundary problem $\left(P_{2 k}, O\right)$ satisfies the Lopatinski-Shapiro condition.

Proof. Let $j \in\{0,1, \ldots, k-1\}$ be given, and note that $\left(n^{a} \nabla_{a}\right)^{j}$ has leading symbol $b_{j}(x, \xi)=C_{j}\left(n^{a} \xi_{a}\right)^{j}$ for some nonzero constant $C_{j}$. Let $x \in \Sigma$ be given, and suppose that $\eta_{a}$ is not parallel to $n_{a}$ at $x$. Then $b_{j}\left(x, \eta_{a}+\tau n_{a}\right)=C_{j}\left(n^{a} \eta_{a}+\tau\right)^{j}$. 
Now let $I$ denote the principal ideal in $\mathbb{C}[\tau]$ generated by $M^{+}$. We claim that the elements $\left[C_{j}\left(n^{a} \eta_{a}+\tau\right)^{j}\right], 0 \leq j \leq k-1$, are linearly independent in $\mathbb{C}[\tau] / I$. To see this, suppose there are constants $C_{0}^{\prime}, \ldots, C_{k-1}^{\prime}$, not all zero, such that $\sum_{j=0}^{k-1} C_{j}^{\prime}\left[C_{j}\left(n^{a} \eta_{a}+\tau\right)^{j}\right]=I$. Then there is a polynomial $h(\tau)$ in $\mathbb{C}[\tau]$ such that

$$
\sum_{j=0}^{k-1} C_{j}^{\prime} C_{j}\left(n^{a} \eta_{a}+\tau\right)^{j}=h(\tau)(\tau-\alpha)^{k} .
$$

But then the left-hand side of (52) is a polynomial of lower degree than the polynomial on the right-hand side of (52). This is a contradiction. The linear independence claim therefore holds, and thus $\left(P_{2 k}, O\right)$ satisfies the Lopatinski-Shapiro condition.

Lemma 6.5. Let $k \in \mathbb{Z}_{>0}$ be given, and suppose that $n, k$, and $(M, \mathbf{c})$ satisfy Condition 5.9. Let $B$ be as in (50). Then the boundary problem $\left(P_{2 k}, B\right)$ satisfies the Lopatinski-Shapiro condition.

Proof. Let $j \in\{0, \ldots, k-1\}$ be given. By Riemannian invariant theory, there exist intrinsic natural differential operators $D_{m}$ on $\Sigma$ (for $0 \leq m \leq j-1$ ) of order at most $j-m$ such that

$$
\delta_{j}^{T}=c_{j}\left(n^{a} \nabla_{a}\right)^{j}+\sum_{m=0}^{j-1} D_{m}\left(n^{a} \nabla_{a}\right)^{m}
$$

along $\Sigma$. Here $c_{j}$ is a nonzero constant. The result then follows by Lemma 6.4, above, and Lemma 6.3 of [6].

Our construction of Dirichlet-to-Neumann operators will also require the following definition:

Definition 6.6. Let $k \in \mathbb{Z}_{>0}$ and a multi-boundary operator $O=\left(O_{1}, \ldots, O_{k}\right)$ be given. Suppose that $O_{1}, \ldots, O_{k}$ are differential operators of orders $m_{1}, \ldots, m_{k}$, respectively, and suppose that $0 \leq m_{1}<\cdots<m_{k}<2 k$. Finally, suppose that for each $i \in\{1, \ldots, k\}$, the operator $O_{i}$ has transverse order $m_{i}$. Then we say that $O$ is a normal multi-boundary operator.

Definition 6.6 is equivalent to the definition of a normal system of boundary operators given on page 34 of [6]. The multi-boundary operator $B$ of (50) is normal.

6.2. The fraction Laplacians. The next theorem gives our construction of conformally invariant Dirichlet-to-Neumann operators.

Theorem 6.7. Let a compact Riemannian conformal manifold with boundary $(M, \Sigma, \mathbf{c})$ be given. Let $k \in \mathbb{Z}_{>0}$ and $\ell \in\{0,1, \cdots, k-1\}$ be given, and suppose that $n, k$, and $(M, \mathbf{c})$ satisfy Condition 5.9. Let $B$ be as in (50), and suppose that the conformal generalised Dirichlet problem $\left(P_{2 k}, B\right)$ has trivial kernel. Then 
there is a well-defined conformally invariant Dirichlet-to-Neumann operator

given by

$$
P_{2 m}^{T, k}: \overline{\mathcal{E}}\left[m-\frac{n-1}{2}\right] \rightarrow \overline{\mathcal{E}}\left[-m-\frac{n-1}{2}\right]
$$

$$
\Gamma\left(\overline{\mathcal{E}}\left[m-\frac{n-1}{2}\right]\right) \ni f \mapsto \delta_{2 k-1-\ell}^{T} u .
$$

Here $m:=k-1 / 2-\ell$, and $u$ solves the conformal generalised Dirichlet problem

$$
P_{2 k} u=0, \quad \delta_{\ell}^{T} u=f, \quad \delta_{j}^{T} u=0 \text { for } j \neq \ell \text { and } 0 \leq j \leq k-1 .
$$

The operator $P_{2 m}^{T, k}$ has leading term $(-\bar{\Delta})^{m}$.

Proof. The problem $\left(P_{2 k}, B\right)$ is properly elliptic, normal, and FSA. It satisfies the Lopatinski-Shapiro condition, and by assumption, it has trivial kernel. Thus by standard theory it follows that (53) has a unique solution $u$ (see e.g. [46, Section 20.1]). Thus the map $f \mapsto \delta_{2 k-1-\ell}^{T} u$ gives a well-defined non-local operator $P_{2 m}^{T, k}: \overline{\mathcal{E}}[m-(n-1) / 2] \rightarrow \overline{\mathcal{E}}[-m-(n-1) / 2]$. By construction this is conformally invariant.

In the case of the dimension $n$ hemisphere, equipped with the standard conformal structure of the round sphere, it is well-known that there is a unique intertwinor between boundary conformal density bundles and with the domain space $\Gamma(\overline{\mathcal{E}}[m-(n-1) / 2])$. This takes values in $\Gamma(\overline{\mathcal{E}}[-m-(n-1) / 2])$ and has leading term $(-\bar{\Delta})^{m}$. See [6]. Thus by the universality of the construction, the final claim follows.

\section{Q-Type Curvatures}

In this section, we define and investigate the $Q$-type curvatures that we discussed in Section 1, above. Our definition is very general. It associates $Q$-type curvatures to the operator families $\delta_{K}, \delta_{K}^{0}$, and $\delta_{J, k}$ of Section 5 and to any other operator families satisfying appropriate properties. This general $Q$-type curvature will exist in all dimensions in which the associated operators exist. The construction of our $Q$-type curvature resembles the construction of Branson's $Q$-curvature in [4], and under conformal change of metric, our $Q$-type curvature will transform in essentially the same way as Branson's $Q$-curvature. Note, however, that the integral of Branson's $Q$-curvature over a compact manifold without boundary is a conformal invariant; we do not claim that our $Q$-type curvatures have this property. Our curvature definition applies to families of natural hypersurface operators $\psi$ which satisfy the following hypotheses:

Hypotheses 7.1. For some $c \in \mathbb{R}, K \in \mathbb{Z}_{>0}$, and $\mathrm{D} \subseteq \mathbb{Z}_{>0}$, the family of operators $\psi: \mathcal{E}[w] \rightarrow \overline{\mathcal{E}}[w-c]$ satisfies Hypotheses [4.1. The operators $\psi$ are conformally invariant and have mass $K$ and order at most $K$. The set $E(\psi)$ of Definition 5.1 may depend on $n$ but is always finite. Finally, in all dimensions $n \in \mathrm{D}, \psi$ annihilates constant densities of weight zero. Specifically, if $f$ is a constant section of $\mathcal{E}[0]$, then $\psi f=0$ along $\Sigma$.

The definition of our $Q$-type curvatures requires some groundwork. 
Definition 7.2. Let $\left\{I_{\beta, g}\right\}_{\beta \in A}$ be the basis for $\mathcal{Q}_{K, c, w}$ that we discussed in Proposition 4.4, above. Let $B$ denote the set of all $\beta \in A$ such that $I_{\beta, g}$ is a composition of the form $O_{\beta, g} \circ \nabla$ for some natural operator $O_{\beta, g}$. Let $C$ denote the set of all $\beta \in A$ such that $I_{\beta, g}$ is a zero-order operator.

Note that $A=B \cup C$ and $B \cap C=\emptyset$. Now recall that if $g$ is any Riemannian metric, then $\xi^{g}$ denotes the scale density associated to $g$.

Definition 7.3. Let $c \in \mathbb{R}$ and a family of conformally invariant natural differential operators $\psi: \mathcal{E}[w] \rightarrow \overline{\mathcal{E}}[w-c]$ be given. For all $w \in \mathbb{R}$ and all $g \in \mathbf{c}$, define $\psi_{g, w}: C^{\infty}(M) \rightarrow C^{\infty}(\Sigma)$ by letting $\psi_{g, w} f=\left(\xi^{g}\right)^{-w+c} \psi\left(\xi^{g}\right)^{w} f$ for all sections $f$ of $\mathcal{E}[0]$. Now let $\left\{I_{\beta, g}\right\}_{\beta \in A}$ be as in Proposition 4.4. For every $w \in \mathbb{R}$ and $\beta \in A$, let $i_{\beta, g}=\left(\xi^{g}\right)^{-w+c} I_{\beta, g}\left(\xi^{g}\right)^{w}$.

In Definition $7.3, \psi_{g, w}$ is conformally covariant of bidegree $(-w,-w+c)$, by Proposition 3.2 . The definition of our $Q$-type curvatures will rely on the following proposition.

Proposition 7.4. Let a family of natural hypersurface operators $\psi$ be given, and suppose that $\psi$ satisfies Hypotheses 7.1. Let notation be as in definitions 7.2 and 7.3. Then for all $w \in \mathbb{R}$,

$$
\psi_{g, w}=\sum_{\beta \in B} f_{\beta}(w, n) i_{\beta, g}+\sum_{\beta \in C} w q_{\beta, n}(w) i_{\beta, g},
$$

where both $f_{\beta}(w, n)$ and $q_{\beta, n}(w)$ are polynomial in $w$ and rational in $n$.

Proof. By Proposition 4.8, $\psi=\sum_{\beta \in B \cup C} f_{\beta}(w, n) I_{\beta, g}$, where each function $f_{\beta}(w, n)$ is polynomial in $w$ and rational in $n$. Let $\beta \in C$ be given. By the polynomial division algorithm from elementary algebra, we may write $f_{\beta}(w, n)=w q_{\beta, n}(w)+$ $r_{\beta, n}$. Here $q_{\beta, n}(w)$ and $r_{\beta, n}$ are rational in $n$, and $q_{\beta, n}(w)$ is polynomial in $w$. Note that $q_{\beta, n}(w)$ and $r_{\beta, n}$ are clearly regular at all $n \in \mathrm{D}$, where $\mathrm{D}$ is as in Hypotheses [7.1. Since $\psi$ annihilates constant densities of weight zero, it follows that $\sum_{\beta \in C} f_{\beta}(0, n) I_{\beta, g}=0$ for all $n \in \mathrm{D}$. Thus $\sum_{\beta \in C} f_{\beta}(0, n)\left[I_{\beta, g}\right]$ is the zero element of $\mathcal{S}_{K, c, 0}$. Here $\left[I_{\beta, g}\right]$ denotes the element of $\mathcal{S}_{K, c, 0}$ determined by $I_{\beta, g}$. Thus $f_{\beta}(0, n)=r_{\beta, n}=0$ for all $\beta \in C$ and all $n \in \mathbb{Z}$, by Proposition 4.6. It follows that $f_{\beta}(w, n)=w q_{\beta, n}(w)$ for $\beta \in C$, all $w \in \mathbb{R}$, and all $n \in \mathrm{D}$. The result follows.

The next definition gives our new general $Q$-type curvatures.

Definition 7.5. Let a family of natural hypersurface operators $\psi$ be given, and suppose that $\psi$ satisfies Hypotheses 7.1. Let notation be as above. We define the following curvature quantity along $\Sigma$ :

$$
Q_{g}(\psi):=-\sum_{\beta \in C} q_{\beta, n}(0) i_{\beta, g} .
$$

Remark 7.6. The curvature quantity $Q_{g}(\psi)$ is a family of hypersurface invariants parametrised by the dimension $n$. We will only be interested in the values of $Q_{g}(\psi)$ at points $p \in \Sigma$, and $Q_{g}(\psi)$ is uniquely determined at such points. Note, however, 
that the right-hand side of (55) is defined on an open neighbourhood of $M$; this will be important in the proof of Proposition 7.15, below.

The following proposition describes a transformation rule for $Q_{g}(\psi)$ under conformal change of metric.

Proposition 7.7. Let $\psi$ be as in Definition 7.5, and let notation be as above. Then

$$
e^{c \Upsilon} Q_{\hat{g}}(\psi)=Q_{g}(\psi)+\psi_{g, 0} \Upsilon .
$$

If we view $Q_{g}(\psi)$ as a density of weight $-c$, then the transformation rule becomes

$$
Q_{\hat{g}}(\psi)=Q_{g}(\psi)+\psi \Upsilon .
$$

Proof. We apply $e^{(-w+c) \Upsilon} \psi_{\hat{g}, w}$ to the constant function 1. By conformal covariance, we obtain

$$
\begin{aligned}
& e^{(-w+c) \Upsilon} \sum_{\beta \in C} w q_{\beta, n}(w) i_{\beta, \widehat{g}}= \\
& \quad-w e^{-w \Upsilon} \sum_{\beta \in B} f_{\beta}(w, n) i_{\beta, g} \Upsilon+w^{2} e^{-w \Upsilon} \ell_{g}(w, n, \Upsilon) \\
& \quad+w e^{-w \Upsilon} \sum_{\beta \in C} q_{\beta, n}(w) i_{\beta, g} .
\end{aligned}
$$

Here $\ell_{g}(w, n, \Upsilon)$ is defined as follows. Let $\beta \in B$ be given. Then

$$
i_{\beta, g} e^{-w \Upsilon}=e^{-w \Upsilon}\left(-w i_{\beta, g} \Upsilon+w^{2} k_{\beta, g}(w, \Upsilon)\right),
$$

where $k_{\beta, g}(w, \Upsilon)$ is polynomial in $w$. Let $\ell_{g}(w, n, \Upsilon)=\sum_{\beta \in B} f_{\beta}(w, n) k_{\beta, g}(w, \Upsilon)$. Now divide (56) by $-w e^{-w \Upsilon}$ to obtain

$$
\begin{aligned}
& -e^{c \Upsilon} \sum_{\beta \in C} q_{\beta, n}(w) i_{\beta, \widehat{g}}= \\
& \quad \sum_{\beta \in B} f_{\beta}(w, n) i_{\beta, g} \Upsilon-w \ell_{g}(w, n, \Upsilon)-\sum_{\beta \in C} q_{\beta, n}(w) i_{\beta, g} .
\end{aligned}
$$

This equation holds in the case $w=0$, since both sides are polynomial in $w$. In this case, we may use (54) to identify the presence of $\psi_{g, 0}$ on the right-hand side of (57), and we see that the proposition holds.

We now apply Definition 7.5 to the operator families $\delta_{K}, \delta_{K}^{0}$, and $\delta_{J, k}$.

Proposition 7.8. Let $K \in \mathbb{Z}_{>0}$ be given, and let $\psi: \mathcal{E}[w] \rightarrow \overline{\mathcal{E}}[w-K]$ denote the $\delta_{K}$ operator family of Lemma 5.4. as acting on densities, the $\delta_{K}^{0}$ family of Theorem 5.12, or the $\delta_{J, k}$ family of Theorem 5.16. (In the latter case, $n, k$, and $(M, \mathbf{c})$ must satisfy Condition 5.9, and $J+k$ must equal $K$.) Then $\psi$ satisfies Hypotheses 7.1, and Definition 7.5 gives a $Q$-type curvature $Q_{g}(\psi)$ associated to $\psi$ and a representative $g$ of the conformal structure on $M$.

Proof. Suppose first that $\psi=\delta_{K}$. The proposition then follows trivially, since by (15), the Thomas $D$-operator annihilates constant functions.

The proofs for $\psi=\delta_{K}^{0}$ and $\psi=\delta_{J, k}$ are similar. We will give the proof for $\psi=\delta_{J, k}$ only. We begin by letting $\psi=\delta_{J, k}$. We will consider the family of conformally invariant operators $O_{J, k}: \mathcal{E}[w] \rightarrow \overline{\mathcal{E}}[w-J-k]$ given by $O_{J, k}:=$ $N^{A_{1}} \cdots N^{A_{k}} \delta_{J} P_{A_{1} \cdots A_{k}}$. By Proposition 5.14, we know that $P_{A_{1} \cdots A_{k}}$ is a family of conformally invariant natural operators of mass $k$ given by a universal symbolic 
formula. It follows that $O_{J, k}$ has mass $J+k$ and satisfies Hypotheses 4.1. By propositions 5.10 and 5.14, $P_{A_{1} \cdots A_{k}}$ is given by either

$$
D_{A_{1}} \ldots D_{A_{k}} \quad \text { or } \quad D_{A_{1}} \ldots D_{A_{k}}-X_{A_{1}} \Psi_{A_{2} \cdots A_{k}}{ }^{P Q} D_{P} D_{Q} \text {. }
$$

Here $\Psi^{k}{ }_{A_{2} \cdots A_{k}}^{P Q}$ is as in Proposition 5.10, Thus $O_{J, k}$ annihilates constant functions, and the proposition follows in the case $k \leq J$. We may thus assume that $k>J$.

Let $\mathrm{D}$ denote the set of all dimensions $n$ in which $\delta_{J, k}$ is well-defined. We apply Proposition 4.8 with $\mathrm{D}$ and with $K=c=J+k$. We may write $O_{J, k}=$ $\sum_{\beta \in B \cup C} f_{\beta}(w, n) I_{\beta, g}$, where $I_{\beta, g}, B$, and $C$ are as in Definition 7.2. For each $\beta \in B \cup C, f_{\beta}(w, n)$ is a polynomial in $w$ whose coefficients are real rational functions of $n$. For all $\beta \in C$, we may use polynomial division to symbolically compute functions $s_{\beta, n}(w)$ and $t_{\beta, n}(w)$, such that

$$
f_{\beta}(w, n)=w(n+2 w-2 k) s_{\beta, n}(w)+t_{\beta, n}(w) .
$$

In this polynomial division, we treat $f_{\beta}(w, n)$ and $w(n+2 w-2 k)$ as polynomials in $w$. Thus $s_{\beta, n}(w)$ and $t_{\beta, n}(w)$ are polynomials in $w$ whose coefficients are real rational functions of $n$. These rational functions are regular at all $n \in \mathrm{D}$, since the divisor has leading term $2 w^{2}$. The degree of $t_{\beta, n}(w)$ is at most 1 .

For all $\beta \in B \cup C$, let $\left[I_{\beta, g}\right]$ denote the element of $\mathcal{S}_{J+k, J+k, 0}$ determined by $I_{\beta, g}$. Since $O_{J, k}$ annihilates constant densities of weight zero, it follows that $\sum_{\beta \in C} f_{\beta}(0, n) I_{\beta, g}$ is the zero operator for all $n$ in $\mathrm{D}$. It therefore follows that $\sum_{\beta \in C} f_{\beta}(0, n)\left[I_{\beta, g}\right]$ is the zero element of $\mathcal{S}_{J+k, J+k, 0}$. Thus $f_{\beta}(0, n)=0$ for all $\beta \in C$ and all $n \in \mathbb{Z}$, by Proposition 4.6, so $t_{\beta, n}(0)=0$ for all $\beta \in C$ and all $n \in \mathrm{D}$, by (58).

Now consider any $V_{0} \in \mathcal{E}[0]$. Then $\left(\xi^{g}\right)^{k-n / 2} V_{0} \in \mathcal{E}[k-n / 2]$, so by Theorem 5.16,

$$
\sum_{\beta \in B \cup C} f_{\beta}(k-n / 2, n) I_{\beta, g}\left(\xi^{g}\right)^{k-n / 2} V_{0}=O_{J, k}\left(\xi^{g}\right)^{k-n / 2} V_{0}=0
$$

along $\Sigma$. But $\nabla\left(\left(\xi^{g}\right)^{k-n / 2}\right)=0$, so $\sum_{\beta \in B \cup C} f_{\beta}(k-n / 2, n) I_{\beta, g} V_{0}$ vanishes along $\Sigma$. It then follows that $\sum_{\beta \in B \cup C} f_{\beta}(k-n / 2, n)\left[I_{\beta, g}\right]$ is the zero element of $\mathcal{S}_{J+k, J+k, 0}$. Thus $f_{\beta}(k-n / 2, n)=0$ for all $\beta \in B \cup C$ and for all $n \in \mathbb{Z}$, by Proposition 4.6. so $t_{\beta, n}(k-n / 2)=0$ for all $\beta \in C$ and all $n \in \mathrm{D}$, by (58).

Let $\beta \in C$ and $n \in \mathrm{D} \backslash\{2 k\}$ be given. Then $t_{\beta, n}(w)$ is a polynomial in $w$ with two distinct zeros, so the coefficients of $t_{\beta, n}(w)$ must vanish in dimension $n$. Since $\mathrm{D} \backslash\{2 k\}$ is an infinite set, these coefficients must therefore vanish for all $n \in \mathbb{Z}$. Thus $f_{\beta}(w, n)=w(n+2 w-2 k) s_{\beta, n}(w)$ for all $n \in \mathrm{D}$ and all $w \in \mathbb{R}$.

Similar reasoning shows that for all $\beta \in B$ and all $n \in \mathrm{D}$, we have $f_{\beta}(w, n)=$ $(n+2 w-2 k) h_{\beta, n}(w)$. Here $h_{\beta, n}(w)$ is polynomial in $w$ and rational in $n$. Thus by polynomial continuation in $w$,

$$
\delta_{J, k}=\sum_{\beta \in B} h_{\beta, n}(w) I_{\beta, g}+\sum_{\beta \in C} w s_{\beta, n}(w) I_{\beta, g} .
$$

Thus $\delta_{J, k}$ annihilates constant densities of weight zero. 
Corollary 7.9. For all $K \in \mathbb{Z}_{>0}$, the operator families $\delta_{K}, \delta_{K}^{0}$, and $\delta_{J, k}$ solve Problem 1.2. (In the case of $\delta_{J, k}$, we assume that $n, k$, and $(M, \mathbf{c})$ satisfy Condition 5.9 and that $J+k=K$.)

The following theorem gives higher-order analogues of $P_{3}^{C Q}$ in the conformally flat setting and in the case of general Riemannian metrics.

Theorem 7.10. Let an even integer $n_{0} \geq 4$ be given, and let $K=n_{0}-1$. Then $\delta_{n_{0}-1}^{0}$ and $\delta_{\left(n_{0}-2\right) / 2, n_{0} / 2}$ solve Problem 1.3 in dimension $n=n_{0}$.

Proof. If $n=n_{0}$, then $0 \notin E\left(\delta_{n_{0}-1}^{0}\right)$ and $0 \notin E\left(\delta_{\left(n_{0}-2\right) / 2, n_{0} / 2}\right)$, by corollaries 5.13 and 5.17. So if $n=n_{0}$, then $\delta_{n_{0}-1}^{0}$ and $\delta_{\left(n_{0}-2\right) / 2, n_{0} / 2}$ are critical operators.

The next two theorems give symbolic tractor formulae associated to $Q_{g}\left(\delta_{J, k}\right)$, $Q_{g}\left(\delta_{K}\right)$, and $Q_{g}\left(\delta_{K}^{0}\right)$.

Theorem 7.11. Let $J, k \in \mathbb{Z}_{>0}$ be given, and view $Q_{g}\left(\delta_{J, k}\right)$ as a density of weight $-J-k$. Let $I_{E}=(n-2) Y_{E}-\mathrm{J} X_{E}$. Then there is a family of conformally invariant differential operators $P^{1}{ }_{A_{1} \ldots A_{k}}{ }^{E}: \mathcal{T}_{E}[w] \rightarrow \mathcal{T}_{A_{1} \ldots A_{k}}[w-k+1]$ with the following properties:

- It is well-defined for all $n$ and $(M, \mathbf{c})$ satisfying Condition 5.9.

- It is given by a polynomial in D, W, X, h, and $h^{\#}$ of mass $k-1$ whose coefficients are rational in $n$.

- If $k>J$, then

$$
(n-2 k) Q_{g}\left(\delta_{J, k}\right)=-N^{A_{1}} \cdots N^{A_{k}} \delta_{J} P^{1}{ }_{A_{1} \ldots A_{k}}{ }^{E} I_{E} .
$$

- If $k \leq J$, then

$$
Q_{g}\left(\delta_{J, k}\right)=-N^{A_{1}} \cdots N^{A_{k}} \delta_{J} P^{1}{ }_{A_{1} \ldots A_{k}}{ }^{E} I_{E} .
$$

Proof. Let $P_{A_{1} \ldots A_{k}}$ be as in Proposition 5.14, above, and suppose first that $k>J$. Then by Proposition 5.10, we may write $P_{A_{1} \ldots A_{k}}=P^{1}{ }_{A_{1} \ldots A_{k}}{ }^{E} D_{E}$, where $P^{1}{ }_{A_{1} \ldots A_{k}}{ }^{E}$ is a polynomial in $D, W, X, h$, and $h^{\#}$ whose coefficients are rational in $n$. Let $\mathrm{D}$ denote the set of all dimensions $n$ in which $\delta_{J, k}$ is defined. By Proposition 7.4,

$$
\delta_{J, k, g, w}=\sum_{\beta \in B} f_{\beta}(w, n) i_{\beta, g}+\sum_{\beta \in C} w q_{\beta, n}(w) i_{\beta, g} .
$$

Here notation is as in definitions 7.2 and 7.3 and Proposition 7.4 , By (15),

$$
\begin{gathered}
(n+2 w-2 k) \delta_{J, k, g, w} 1=\sum_{\beta \in C} w(n+2 w-2 k) q_{\beta, n}(w) i_{\beta, g} \\
=\left(\xi^{g}\right)^{-w+J+k} N^{A_{1}} \cdots N^{A_{k}} \delta_{J} P^{1}{ }_{A_{1} \ldots A_{k}}{ }^{E} w \tilde{I}_{E}\left(\xi^{g}\right)^{w},
\end{gathered}
$$

where $\tilde{I}_{E}=(n+2 w-2) Y_{E}-\mathrm{J} X_{E}$. We may use (11), (13), (14), (15), (20), and the rules of Figure 1 to expand the rightmost expression in (60). Then by polynomial continuation in $w$, we find that

$$
\begin{gathered}
-(n+2 w-2 k)\left(\xi^{g}\right)^{-J-k} \sum_{\beta \in C} q_{\beta, n}(w) i_{\beta, g}= \\
-\left(\xi^{g}\right)^{-w} N^{A_{1}} \cdots N^{A_{k}} \delta_{J} P^{1}{ }_{A_{1} \ldots A_{k}}{ }^{E} \tilde{I}_{E}\left(\xi^{g}\right)^{w}
\end{gathered}
$$


for all $w \in \mathbb{R}$. If we let $w=0$ in (61), then (59) follows.

The proof for the case $k \leq J$ is straightforward.

Theorem 7.12. Let $I_{E}$ be as in Theorem 7.11, and for all $K \in \mathbb{Z}_{>0}$, view $Q_{g}\left(\delta_{K}\right)$ and $Q_{g}\left(\delta_{K}^{0}\right)$ as densities of weight $-K$. Then for all $K \in \mathbb{Z}_{\geq 2}$,

$$
Q_{g}\left(\delta_{K}\right)=-N^{A_{1}} \cdots N^{A_{K-1}} \delta_{1} D_{A_{1}} \cdots D_{A_{K-2}} I_{A_{K-1}}
$$

and

$$
\left[\prod_{j=1}^{\left\lfloor\frac{K-1}{2}\right\rfloor}(n-2 K+2 j)\right] Q_{g}\left(\delta_{K}^{0}\right)=-N^{A_{1}} \cdots N^{A_{k-1}} \delta_{1} D_{A_{1}} \cdots D_{A_{K-2}} I_{A_{K-1}} .
$$

In the above equations, if $K=2$, then $D_{A_{1}} \ldots D_{A_{K-2}}$ is the identity operator. On the other hand, $Q_{g}\left(\delta_{1}\right)=H$ and $Q_{g}\left(\delta_{1}^{0}\right)=H$.

Proof. The proof is similar to the proof of Theorem 7.11 .

One may use theorems 7.11 and 7.12 to construct symbolic formulae for $Q_{g}\left(\delta_{J, k}\right)$, $Q_{g}\left(\delta_{K}\right)$, and $Q_{g}\left(\delta_{K}^{0}\right)$. In some cases, this requires a special procedure. For example, consider $Q_{g}\left(\delta_{J, k}\right)$ when $k>J$. By applying (11), (13), (14), (15), (20), and the rules in Figure 1, above, one may convert the right-hand side of (59) into a polynomial in $n_{a}, R_{a b}{ }^{c} d, \boldsymbol{g}_{a b}, \mathbf{g}^{a b}$, the modified mean curvature $G$, and the LeviCivita connection $\nabla$. By commuting covariant derivatives and applying standard tensor identities, one may (in principle) convert this polynomial into an expression of the form $(n-2 k) \psi$, where $\psi$ is again a polynomial of the above type. Thus $(n-2 k) Q_{g}\left(\delta_{J, k}\right)=(n-2 k) \psi$ in all dimensions $n \in \mathrm{D}$, where $\mathrm{D}$ is the set of all dimensions in which $\delta_{J, k}$ is defined. Now let $K=J+k$, and note that $Q_{g}\left(\delta_{J, k}\right)$ and $\psi$ define differential operators of order zero. These operators are elements of $\mathcal{R}_{K, K, 0}$, and they are equal in all dimensions $n \in \mathrm{D} \backslash\{2 k\}$. Thus $Q_{g}\left(\delta_{J, k}\right)=\psi$ in dimension $n=2 k$, by Proposition 4.9.

We have used (59) and the above procedure to find an explicit symbolic formula for $Q_{g}\left(\delta_{J, k}\right)$ in a few specific low-order cases.

Next we shall see that the behaviour of the curvatures of $\delta_{K}$ and $\delta_{J, k}$, as defined by (55), or equivalently by theorems 7.11 and 7.12 , varies strongly according to dimension parity. This suggests that we introduce some terminology.

Definition 7.13. Let $c \in \mathbb{R}, K \in \mathbb{Z}_{\geq 0}$, and a Riemannian hypersurface invariant $T^{g} \in \overline{\mathcal{E}}[-c]$ be given. Suppose that $T^{g}$ has a conformal transformation of the form $T^{\widehat{g}}=T^{g}+P \Upsilon$, where $P: \mathcal{E}[0] \rightarrow \overline{\mathcal{E}}[-c]$ is a conformally invariant natural hypersurface differential operator of order and transverse order $K$. Then we shall say that $T^{g}$ is a hypersurface T-curvature (or transverse curvature) of order $K$. We shall call $(P, T)$ a T-curvature pair.

This terminology is inspired by the Chang-Qing pair $\left(P_{3}^{C Q}, T^{C Q}\right)$ which we discussed in Section 1, above. This pair is a $T$-curvature pair in the sense of Definition 7.13. This is very different from the Branson $Q$-curvature of the hypersurface, so we shall say " $Q$-curvature" for quantities closer to that behaviour. More precisely, we make the following definition. 
Definition 7.14. Let $c \in \mathbb{R}, K \in \mathbb{Z}_{>0}$, and a Riemannian hypersurface invariant $Q^{g} \in \overline{\mathcal{E}}[-c]$ be given. Suppose that $Q^{g}$ has a conformal transformation of the form $Q^{\widehat{s}}=Q^{g}+P \Upsilon$, where $P: \mathcal{E}[0] \rightarrow \overline{\mathcal{E}}[-c]$ is a conformally invariant natural hypersurface differential operator of order $K$ and transverse order less than $K$. Then we shall say that $Q^{g}$ is a hypersurface $Q$-curvature of order $K$. We shall call $(P, Q)$ a $Q$-curvature pair.

It is clear that the $T$-curvatures of Definition 7.13 are recovered from the operators $\delta_{K}, \delta_{K}^{0}$, and $\delta_{J, k}$ in all cases in which these operators are defined and the weight 0 is not in the respective exceptional list $E\left(\delta_{K}\right), E\left(\delta_{K}^{0}\right)$, or $E\left(\delta_{J, k}\right)$. Theorem 1.6, above, thus follows from (36) and Theorem 5.16.

Let notation be as in Theorem 1.6, and let an even integer $n_{0} \geq 4$ be given. Then in dimension $n=n_{0}$, we should think of the critical pair $\left(\delta_{\left(n_{0}-2\right) / 2, n_{0} / 2}, T_{n_{0}-1}\right)$ as an analogue of the Chang-Qing $\left(P_{3}^{C Q}, T^{C Q}\right)$-pair. Note that $T_{1}^{g}$ is simply $H^{g}$, the mean curvature of the hypersurface, so the $T$-curvatures may also be thought of as (conformal geometry-inspired) higher-order generalisations of the mean curvature. This also suggests an interesting application of the $T$-curvatures, which we describe as follows.

Proposition 7.15. Suppose that $n$ is even, $\Sigma$ is closed as a subset of $M$, and $\Sigma$ and $M$ are orientable. For all $i \in \mathbb{Z}_{>0}$, let $T_{i}^{g}$ be as in Theorem 1.6. Finally, let $g \in \mathbf{c}$ be given. Then for all $m \in \mathbb{Z}_{>0}$, there is a metric $\widehat{g} \in \mathbf{c}$ such that $\widehat{g}$ induces $\bar{g}$ and

$$
T_{1}^{\widehat{g}}=T_{2}^{\widehat{g}}=\cdots=T_{m}^{\widehat{g}}=0 \quad \text { along } \Sigma .
$$

Along $\Sigma$, the metric $\widehat{g}$ is uniquely determined by $\bar{g}$ to order $m$.

The above results also hold if $\Sigma$ is not closed as a subset of $M$. In this case, however, we only claim that the metric $\widehat{g}$ is defined on an open neighbourhood $O$ of $\Sigma$ in $M$. Thus $\widehat{g}=e^{2 \Upsilon} g$ on $O$ for some $\Upsilon \in C^{\infty}(O)$. The neighbourhood $O$ may depend on $m$.

Proof. Suppose first that $\Sigma$ is closed. For any $p \in \Sigma$, we may define local coordinates $\left(x^{1}, \ldots, x^{n}\right)$ on a neighbourhood of $p$ in $M$ such that $x^{n}$ is a local defining function for $\Sigma$. Suppose that $\left(y^{1}, \ldots, y^{n}\right)$ is any other local coordinate system, and suppose that $y^{n}$ is also a local defining function for $\Sigma$. We may assume that $\partial y^{n} / \partial x^{n}>0$ at all points of $\Sigma$ at which both coordinate systems are defined; this follows from the fact that $M$ and $\Sigma$ are both orientable. By using such coordinate systems along with an appropriate partition of unity, one can show that for some open neighbourhood $U$ of $\Sigma$, there is a defining function $t$ for $\Sigma$ on $U$.

For any $\Upsilon, \Upsilon^{\prime}$, and $\Upsilon^{\prime \prime}$ in $\mathcal{E}$, we will let $\widehat{g}=e^{2 \Upsilon} g, \widehat{g}^{\prime}=e^{2 \Upsilon^{\prime}} g$, and $\widehat{g}^{\prime \prime}=e^{2 \Upsilon^{\prime \prime}} g$. For all $i \in \mathbb{Z}_{>0}$, let $\psi_{i}$ denote the operator corresponding to $T_{i}^{g}$ in Theorem 1.6.

To establish the existence of the desired $\widehat{g}$, we proceed by induction on $m$. Let $s \in \mathbb{Z}_{\geq 0}$ be given. If $s=0$, let $\Upsilon \equiv 0$ on $M$. Otherwise, suppose there is an $\Upsilon \in \mathcal{E}$ such that $\Upsilon$ vanishes along $\Sigma$, and (62) holds in the $m=s$ case. Since $0 \notin E\left(\psi_{s+1}\right)$, there is an open set $V \subseteq M$ such that (1) $\Sigma \subseteq V \subseteq U$, and (2) $\psi_{s+1} t^{s+1}$ is nonzero on $V$. Choose any $\varphi \in \mathcal{E}$ with support in $V$ such that $\varphi \equiv 1$ on $\Sigma$, and let 
$\Upsilon^{\prime}=\Upsilon-t^{s+1} \varphi T_{s+1}^{\widehat{\mathrm{g}}} /\left(\psi_{s+1} t^{s+1}\right)$. Then $T_{1}^{\widehat{\mathrm{g}}^{\prime}}=T_{2}^{\widehat{\mathrm{g}}^{\prime}}=\cdots=T_{s+1}^{\widehat{\mathrm{g}}^{\prime}}=0$ along $\Sigma$, by Proposition 7.7 .

To establish uniqueness, it suffices to show that for every $m \in \mathbb{Z}_{>0}$ and every $\Upsilon^{\prime}, \Upsilon^{\prime \prime} \in \mathcal{E}$, if $\widehat{g}=\widehat{g}^{\prime}$ and $\widehat{g}=\widehat{g}^{\prime \prime}$ induce $\bar{g}$ and satisfy (62), then $\Upsilon^{\prime \prime}-\Upsilon^{\prime}$ vanishes to order $m$ along $\Sigma$. We again proceed by induction on $m$. Let $s \in \mathbb{Z}_{\geq 0}$ be given. If $s>0$, suppose that the desired statement holds in the case $m=s$. Let $\Upsilon^{\prime}, \Upsilon^{\prime \prime} \in \mathcal{E}$ be given, and suppose that $\widehat{g}=\widehat{g}^{\prime}$ and $\widehat{g}=\widehat{g}^{\prime \prime}$ induce $\bar{g}$ and satisfy (62) in the $m=s+1$ case. Then $\Upsilon^{\prime \prime}-\Upsilon^{\prime}$ vanishes to order $s$ along $\Sigma$. But $\widehat{g}^{\prime \prime}=e^{2\left(\Upsilon^{\prime \prime}-\Upsilon^{\prime}\right)} \widehat{g}^{\prime}$. Thus $\psi_{s+1}\left(\Upsilon^{\prime \prime}-\Upsilon^{\prime}\right)=0$ along $\Sigma$, by Proposition 17.7, Recall that $0 \notin E\left(\psi_{s+1}\right)$. So if we work in local coordinates of the type that we discussed above, a short argument shows that $\Upsilon^{\prime \prime}-\Upsilon^{\prime}$ vanishes to order $s+1$ along $\Sigma$.

If $\Sigma$ is not closed, the proof is similar to the above proof.

Remark 7.16. In even dimensions, Proposition 7.15 generalises a previous result which has occasionally been used in the literature of general relativity. For even $n \geq 4$, Proposition 7.15 gives a way of normalising the conformal scale so that in the new scale, $\Sigma$ is minimal (i.e. $H=0$ ) and also satisfies related higher-order conditions. By Theorem 7.18, below, similar results hold in the case in which $M$ is odd-dimensional, but the range of available $T$-curvatures is smaller. A statement of a zero-order version of these results appears in Proposition 4.1 of [29].

In the proof of Theorem 7.18 , below, we will use the following lemma to determine the order of $\delta_{K}: \mathcal{E}[0] \rightarrow \overline{\mathcal{E}}[-K]$ in odd dimensions.

Lemma 7.17. Let $K \in \mathbb{Z}_{>0}$ and $w \in \mathbb{R}$ be given, and suppose that $V$ is any section of $\mathcal{T}^{\Phi}[w]$. Then

$$
\delta_{K} V=\left[\prod_{i=1}^{K-1}(n+2 w-2 i)\right] \nabla_{n}^{K} V+\sum_{\beta \in A} C_{\beta}(n, w) P_{\beta} V+\text { lots }
$$

Here $A$ is a finite index set, and for each $\beta \in A, C_{\beta}(n, w)$ is a real polynomial in $n$ and $w$. For each $\beta \in A$, there is an $\ell \in \mathbb{Z}_{>0}$ such that $P_{\beta}=n^{i_{1}} \cdots n^{i_{K-2 \ell}} O_{i_{1} \ldots i_{K-2 \ell}}^{\beta}$, where $O^{\beta}$ is the composition of the operators $\nabla_{i_{1}}, \ldots, \nabla_{i_{K-2 \ell}}$, and $\ell$ copies of $\Delta$, in some order. Finally, lots can be given by a symbolic formula which satisfies Hypotheses 5.3 .

Proof. The lemma follows from (11), (15), (20), Figure 1, lemmas 5.5 and 5.7, and an inductive argument.

Theorem 7.18. Let $K \in \mathbb{Z}_{>0}$ be given, and suppose that $\operatorname{dim}(M)=n$ is odd. Let $\psi: \mathcal{E}[w] \rightarrow \overline{\mathcal{E}}[w-K]$ denote the operator family $\delta_{K}$ of Lemma 5.4 or the operator family $\delta_{J, k}$ of Theorem 5.16 . (In the latter case $J+k$ must equal $K$.) If

$$
\frac{n+1}{2} \leq K \leq n-1
$$

then there is a canonical $Q$-curvature pair $\left(\psi, Q_{K}^{g}\right)$ of order $K$. Here $Q_{K}^{g}:=Q_{g}(\psi)$. In particular, in the critical-order case in which $K=n-1$, we obtain a $Q$-curvature 
$\operatorname{pair}\left(\psi, Q_{n-1}^{g}\right)$. If

$$
K \leq \frac{n-1}{2} \text { or } n \leq K
$$

then there is a $T$-curvature pair $\left(\psi, T_{K}^{g}\right)$ of order $K$. Here $T_{K}^{g}:=Q_{g}(\psi)$.

Proof. Suppose first that $K \leq(n-1) / 2$ or $n \leq K$. Then $0 \notin E\left(\delta_{K}\right)$, by (36) . Thus if $\psi=\delta_{J, k}$, then $0 \notin E(\psi)$, by Theorem 5.16, Thus $\psi: \mathcal{E}[0] \rightarrow \overline{\mathcal{E}}[-K]$ has order and transverse order $K$, and $Q_{g}(\psi)$ is a $T$-curvature.

Now suppose instead that $(n+1) / 2 \leq K \leq n-1$. Then $0 \in E\left(\delta_{K}\right)$, by (36). For the case in which $\psi=\delta_{J, k}$, note that $k-n / 2 \neq 0$, since $n$ is odd; thus $0 \in E\left(\delta_{J, k}\right)$, by Theorem 5.16. So to complete the proof, it suffices to show that $\psi: \mathcal{E}[0] \rightarrow \overline{\mathcal{E}}[-K]$ has order $K$.

Let $V \in \mathcal{E}[0]$ be given, and apply Lemma 7.17. By commuting covariant derivatives, if necessary, we may write

$$
\delta_{K} V=\left[\prod_{i=1}^{K-1}(n-2 i)\right] \nabla_{n}^{K} V+\sum_{i=1}^{\lfloor K / 2\rfloor} C_{i}^{\prime}(n) \nabla_{n}^{K-2 i} \Delta^{i} V+\text { lots } .
$$

Here $C_{i}^{\prime}(n)$ is a real polynomial in $n$. It follows that $\delta_{K}$, as acting on $V$, has order $K$. Now suppose that $\psi=\delta_{J, k}$. Since $w=0$, it follows from Theorem 5.16 that $\psi$ is a nonzero multiple of $N^{A_{1}} \cdots N^{A_{k}} \delta_{J} P_{A_{1} \ldots A_{k}}$. But by (47) and the discussion following (47), $N^{A_{1}} \cdots N^{A_{k}} \delta_{J} P_{A_{1} \ldots A_{k}}=\delta_{K}+$ lots. Thus $\psi: \mathcal{E}[0] \rightarrow \overline{\mathcal{E}}[-K]$ has order $K$.

\section{EXAMPLES}

By using the constructions of Lemma 5.4 and theorems 5.12 and 5.16 , one may construct explicit formula for $\delta_{K}, \delta_{K}^{0}$, and $\delta_{J, k}$ of the type described in Hypotheses 4.1. One may also use theorems 7.11 and 7.12 to construct similar explicit formulae for $Q_{g}\left(\delta_{K}\right), Q_{g}\left(\delta_{K}^{0}\right)$, and $Q_{g}\left(\delta_{J, k}\right)$. The construction of such formulae for $\delta_{J, k}$ and $Q_{g}\left(\delta_{J, k}\right)$ uses the algorithm of [35] for constructing tractor formulae for the GJMS operators $P_{2 k}$. We have constructed explicit formulae of the above types in a few low-order cases. In this section, we discuss these constructions and give some of the resulting explicit formulae. In some cases, we have incorporated the trace-free second fundamental form $\stackrel{o}{L}_{a b}$ into the formulae. In constructing the explicit formulae, we used the results described in Section 3, above. In many cases, we also used Mathematica, together with J. Lee's Ricci package [51, 62].

We will need specific tractor formulae for two of the operator families of Proposition [5.14, By [35], we have $P_{A B}=D_{A} D_{B}$ and

$$
P_{A B C}=D_{A} D_{B} D_{C}-\frac{2}{n-4} X_{A} W_{B}^{E}{ }_{C}^{F} D_{E} D_{F} .
$$

8.1. Second-Order Operator. The operator family $\delta_{2}:\left.\mathcal{T}^{\Phi}[w] \rightarrow \mathcal{T}^{\Phi}[w-2]\right|_{\Sigma}$ of Lemma 5.4 is given by (44). By Definition 7.5,

$$
Q_{g}\left(\delta_{2}\right)=\mathrm{J}+(n-2) H^{2}-(n-2) n^{a} n^{b} \mathrm{P}_{a b} .
$$




$$
\begin{aligned}
& (n+2 w-5) \delta \square f \\
& -(n+2 w-2)\left(\bar{\square} \delta f-\frac{2}{3(n-3)} \ell_{2} f+\frac{3 n-5}{4(n-2)} \stackrel{o}{L}_{a b} \stackrel{o}{L}^{a b} \delta f\right. \\
& \left.-\frac{n-5}{2(n-3)} \stackrel{o}{L}_{a b} \stackrel{o}{L}_{c}^{a} \stackrel{o}{L}^{b c} f-\frac{n-5}{2(n-3)} n^{a} n^{b} \stackrel{o}{L}_{c d} C_{a}{ }^{c} b^{d} f\right) \\
& +(n+2 w-2)(n+2 w-5)\left(-H n^{a} n^{b} \nabla_{a} \nabla_{b} f-\frac{2(n+2 w-1)}{3} \stackrel{o}{L}^{a b} \nabla_{a} \nabla_{b} f\right. \\
& +\frac{7-4 n-12 w+4 n w+8 w^{2}}{3}\left(\bar{\nabla}_{a} H\right) \nabla^{a} f+\frac{3(w-1)}{2} H^{2} n^{a} \nabla_{a} f \\
& +\frac{-7+4 n+12 w-4 n w-8 w^{2}}{3} n^{a} n^{b} n^{c} \mathrm{P}_{a b} \nabla_{c} f+\frac{2\left(5-2 n-6 w+2 n w+4 w^{2}\right)}{3} n^{a} \mathrm{P}_{a}{ }^{b} \nabla_{b} f \\
& -\frac{1}{4(n-2)} \stackrel{\circ}{L}_{a b} \stackrel{o}{L}^{a b} n^{c} \nabla_{c} f-\frac{w\left(5-2 n-6 w+2 n w+4 w^{2}\right)}{3(n-3)}(\bar{\Delta} H) f-\frac{w(w-1)}{2} H^{3} f \\
& +\frac{w\left(9+2 n-2 n^{2}-6 n w+2 n^{2} w+4 n w^{2}\right)}{3(n-3)} H n^{a} n^{b} \mathrm{P}_{a b} f \\
& +\frac{w\left(5-2 n-6 w+2 n w+4 w^{2}\right)}{3(n-3)} n^{a} n^{b} n^{c}\left(\nabla_{a} \mathrm{P}_{b c}\right) f-\frac{w\left(5-2 n-6 w+2 n w+4 w^{2}\right)}{3(n-3)} H \mathrm{~J} f \\
& -\frac{w\left(5-2 n-6 w+2 n w+4 w^{2}\right)}{3(n-3)} n^{a}\left(\nabla_{a} \mathrm{~J}\right) f-\frac{2 w(n+2 w-2)(n+2 w-4)}{3(n-3)} \stackrel{o}{L}^{a b} \mathrm{P}_{a b} f \\
& -\frac{w\left(-31+33 n-8 n^{2}+48 w-40 n w+8 n^{2} w-32 w^{2}+16 n w^{2}\right)}{12(n-2)(n-3)} H \stackrel{\circ}{L}_{a b} \stackrel{o}{L}^{a b} f \\
& +\frac{(2 w+1)\left(9-3 n-8 w+2 n w+4 w^{2}\right)}{6(n-3)^{2}} \stackrel{o}{L}_{a b} L^{a}{ }_{c}{ }_{c}^{o}{ }^{b c} f \\
& \left.+\frac{(2 w+1)\left(9-3 n-8 w+2 n w+4 w^{2}\right)}{6(n-3)^{2}} n^{a} n^{b} \stackrel{o}{L}_{c d} C_{a}{ }^{c} b^{d} f\right)
\end{aligned}
$$

Figure 2. $\delta_{1,2} f$ for dimensions $n \geq 4$ and $f \in \mathcal{E}[w]$

From (36), it follows that $E\left(\delta_{2}\right)=\{(3-n) / 2\}$, so for all $n \geq 4, Q_{g}\left(\delta_{2}\right)$ is a hypersurface $T$-curvature in the sense of Definition 7.13. In the $n=3$ case, $Q_{g}\left(\delta_{2}\right)$ is a $Q$-curvature in the sense of Definition 7.14.

In [43], D.H. Grant discusses a related family of conformally invariant secondorder hypersurface differential operators $\bar{D}^{A} \Pi_{A}{ }^{B} D_{B}: \mathcal{E}[w] \rightarrow \overline{\mathcal{E}}[w-2]$. For any $w \in \mathbb{R}$ and any $f \in \mathcal{E}[w]$,

$$
\begin{aligned}
& \bar{D}^{A} \Pi_{A}{ }^{B} D_{B} f=(n+w-3)((n+2 w-2) \bar{\square} f \\
& \left.\quad+(n+2 w-3)\left((n+2 w-2)\left(H n^{a} \nabla_{a} f-\frac{w}{2} H^{2} f\right)-\square f\right)\right),
\end{aligned}
$$

by [43]. A computation shows that

$$
(n+w-3) \delta_{2} f+\bar{D}^{A} \Pi_{A}^{B} D_{B} f=-\frac{w(n+w-3)(n+2 w-2)}{2(n-2)} \stackrel{o}{L}_{a b} \stackrel{o}{L}^{a b} f .
$$

If we divide by $n+w-3$, polynomial continuation shows that

$$
\delta_{2} f=-\bar{\square} f+\frac{n-3}{4(n-2)} \stackrel{o}{L}_{a b} \stackrel{o}{L}^{a b} f, \quad \text { for } f \in \mathcal{E}\left[\frac{3-n}{2}\right] .
$$

In this case, $\square$ is the intrinsic Yamabe operator.

8.2. Third-Order Operator. Consider $\delta_{1,2}: \mathcal{E}[w] \rightarrow \overline{\mathcal{E}}[w-3]$. This family of operators is well-defined in all ambient dimensions $n \geq 3$, and from Theorem 5.16 , it follows that $E\left(\delta_{1,2}\right)=\{(5-n) / 2\}$. By Theorem 5.16, $(n+2 w-4) \delta_{1,2}=$ $N^{A} N^{B} \delta D_{A} D_{B}$. For $n \geq 4$, symbolic computations show that $\delta_{1,2}$ is given by the formula in Figure 2. Here $\ell_{2}$ is the conformally invariant operator $L^{A B} D_{A} D_{B}$; 
additional symbolic computations show that for all $w \in \mathbb{R}$ and all $f \in \mathcal{E}[w]$,

$$
\begin{aligned}
& \ell_{2} f=(n+2 w-2)(n+2 w-4)\left((n-3) L^{a b} \nabla_{a} \nabla_{b} f\right. \\
& \quad-2(n-3)(w-1)\left(\bar{\nabla}_{a} H\right) \nabla^{a} f+w(w-1)(\bar{\Delta} H) f \\
& \quad-n(w-1) w H n^{a} n^{b} \mathrm{P}_{a b} f-w(w-1) n^{a} n^{b} n^{c}\left(\nabla_{a} \mathrm{P}_{b c}\right) f \\
& \quad+w(w-1) H \mathrm{~J} f+2(n-3)(w-1) n^{a} n^{b} n^{c} \mathrm{P}_{a b} \nabla_{c} f \\
& \quad+w(w-1) n^{a}\left(\nabla_{a} \mathrm{~J}\right) f-2(n-3)(w-1) n_{a} \mathrm{P}^{a b} \nabla_{b} f \\
& \quad+w(n+2 w-5) \mathrm{P}_{a b} L^{a b} f+w(w-1) H \stackrel{o}{L}_{a b} \stackrel{o}{a}^{a b} f \\
& \left.\quad-\frac{w(w-1)}{n-3} \stackrel{o}{L}_{a b} \stackrel{o}{L}^{a}{ }_{c} \stackrel{o}{ }^{b c} f-\frac{w(w-1)}{n-3} n^{a} n^{b} \stackrel{o}{L}_{c d} C_{a}^{c}{ }^{d} f\right) .
\end{aligned}
$$

Figure 2 shows that for $f \in \mathcal{E}[1-n / 2]$, we have $\delta_{1,2} f=-3 \delta \square f$. Thus $\delta_{1,2}$ : $\mathcal{E}[1-n / 2] \rightarrow \overline{\mathcal{E}}[-2-n / 2]$ is the composition of two conformally invariant operators. Similarly, Figure 2 shows that for $f \in \mathcal{E}[5 / 2-n / 2]$,

$$
\begin{aligned}
& \delta_{1,2} f=-3 \bar{\square} \delta f+\frac{2}{n-3} \ell_{2} f-\frac{3(3 n-5)}{4(n-2)} \stackrel{o}{L}_{a b} \stackrel{o}{L}^{a b} \delta f \\
& \quad+\frac{3(n-5)}{2(n-3)} \stackrel{o}{L}_{a b} \stackrel{o}{L}^{a}{ }_{c} \stackrel{o}{L}^{b c} f+\frac{3(n-5)}{2(n-3)} n^{a} n^{b} \stackrel{o}{L}_{c d} C_{a}{ }^{c}{ }_{b}^{d} f .
\end{aligned}
$$

Here $\bar{\square}$ acts on a density of weight $1-(n-1) / 2$. Thus $\bar{\square}$ is conformally invariant in this case, and we have again expressed $\delta_{1,2}$ in terms of conformally invariant operators. We gave a symbolic formula for $Q_{g}\left(\delta_{1,2}\right)$ in (1).

In Section 11, above, we discussed the operators $\delta_{3}^{G}$ of [43]. These operators exist in all dimensions $n \geq 4$ and map $\mathcal{E}[(4-n) / 2]$ to $\overline{\mathcal{E}}[(-2-n) / 2]$. The leading term of $\delta_{3}^{G}$ equals the leading term of $\delta_{1,2}: \mathcal{E}[(4-n) / 2] \rightarrow \overline{\mathcal{E}}[(-2-n) / 2]$, up to a nonzero scale.

Now suppose that $M$ is a compact 4-manifold with boundary $\Sigma$, and suppose that the unit normal vector $n^{a}$ points inward. Symbolic computations using [51] and [62] show that for all $f \in \mathcal{E}[0], \delta_{1,2}$ and the third-order boundary operator $P_{3}^{C Q}$ of [16] satisfy the following:

$$
P_{3}^{C Q} f=-\frac{1}{2} \delta_{1,2} f-L^{A B} \bar{D}_{B} \bar{D}_{A} f+\stackrel{o}{L}_{a b} \stackrel{o}{a b}^{a b} \delta .
$$

8.3. Fourth-Order Operator. Consider $\delta_{1,3}: \mathcal{E}[w] \rightarrow \overline{\mathcal{E}}[w-4]$. By Theorem 5.16, together with (63),

$$
(n+2 w-6) \delta_{1,3} f=N^{A} N^{B} N^{C} \delta\left(D_{A} D_{B} D_{C} f-\frac{2}{n-4} X_{A} W_{B}{ }^{E}{ }_{C}^{F} D_{E} D_{F} f\right)
$$

for all $f \in \mathcal{E}[w]$. For $n \geq 5$, symbolic computations show that $\delta_{1,3}$ has the form given in Figure 3. In this figure, $K\left(L^{A B}, L^{C D}\right)$ is a scalar invariant which we define as follows. First, note that by (15) and Figure 1, $\left(\bar{D}_{A} L_{B C}\right) \bar{D}^{A} L^{B C}$ contains a factor $(n-5)$. Divide the symbolic formula for $\left(\bar{D}_{A} L_{B C}\right) \bar{D}^{A} L^{B C}$ by $(n-5)$, and let $K\left(L^{A B}, L^{C D}\right)$ be defined by the resulting symbolic formula. The quantity defined in this way is conformally invariant in all dimensions $n \geq 5$. In dimension 5 , this follows from dimensional continuation.

Inspection of Figure 3 reveals the particular form that $\delta_{1,3} f$ takes when the weight $w$, of $f$, is equal to $2-n / 2,1-n / 2,5 / 2-n / 2$, or $7 / 2-n / 2$. In each 


$$
\begin{aligned}
& \frac{(n+2 w-2)(n+2 w-5)(n+2 w-7)}{2}\left(-Y^{A} \square D_{A} f\right) \\
& +\frac{(n+2 w-4)(n+2 w-5)(n+2 w-7)}{10} \delta_{2} \square f \\
& -\frac{(n+2 w-2)(n+2 w-4)(n+2 w-7)}{2}\left(-\bar{Y} A \bar{\square} \bar{D}_{A} f+\frac{4}{3(n-3)} L^{A B} \delta D_{A} D_{B} f\right. \\
& -\frac{2(n-4)}{3(n-3)} \stackrel{\circ}{L} a \stackrel{o}{L}^{a b} \delta_{2} f-\frac{n-7}{2(n-3)^{2}(n-2)} L^{A B} \bar{\square} L_{A B} f \\
& -\frac{2(n-5)}{3(n-3)(n-4)} N^{C} N^{D} W^{A}{ }_{C}{ }^{B}{ }_{D} D_{A} D_{B} f+\frac{2(n-1)}{3(n-3)^{3}} L^{A}{ }_{C} L^{C B} D_{A} D_{B} f
\end{aligned}
$$

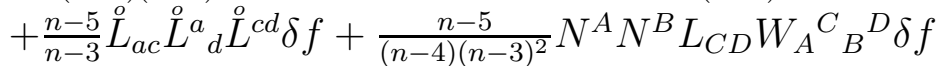

$$
\begin{aligned}
& +\frac{4}{3(n-4)(n-3)} N^{B} L_{C D} W^{A C}{ }_{B}^{D} D_{A} f-\frac{1}{2(n-3)^{2}(n-2)} K\left(L^{A B}, L^{C D}\right) f \\
& +\frac{n-5}{(n-3)^{2}} \stackrel{o}{L}_{a b} \stackrel{o}{L}_{c d} \stackrel{o}{L}^{a c} \stackrel{o}{L}^{b d} f+\frac{n-5}{(n-3)^{2}} n^{a} n^{b} n^{c} n^{d} C_{a e b i} C_{c}{ }^{e}{ }^{i} f \\
& \left.+\frac{(n-5)\left(n^{3}-15 n^{2}+51 n-53\right)}{16(n-3)^{2}(n-2)^{2}} \stackrel{o}{L}_{a b} \stackrel{o}{L}^{a b} \stackrel{o}{L}_{c d} \stackrel{o}{L}^{c d} f+\frac{2(n-5)}{(n-3)^{2}} n^{a} n^{b} \stackrel{o}{L}_{c d} \stackrel{o}{L}^{c}{ }_{e} C_{a}{ }^{d}{ }^{e} f\right) \\
& -\frac{(n+2 w-2)(n+2 w-4)(n+2 w-5)}{10}\left(\bar{\square} \delta_{2} f-\frac{4}{3(n-3)} L^{A B} \delta D_{A} D_{B} f\right. \\
& +\frac{7 n-13}{4(n-2)} \stackrel{o}{L}_{a b} \stackrel{o}{L}^{a b} \delta_{2} f+\frac{2}{3(n-4)} N^{C} N^{D} W^{A}{ }_{C}^{B}{ }_{D} D_{A} D_{B} f \\
& -\frac{2}{3(n-3)^{2}} L^{A}{ }_{C} L^{C B} D_{A} D_{B} f-\frac{4}{(n-4)(n-3)} N^{A} L_{B C} W^{E B}{ }_{A}^{C} D_{E} f \\
& \left.-\frac{5(n-5)}{n-3} \stackrel{\circ}{L_{b c}} \stackrel{o}{L}_{d}^{b} \stackrel{o}{L}^{c d} \delta f-\frac{5(n-5)}{(n-4)(n-3)^{2}} N^{A} N^{B} L_{C D} W_{A}{ }_{B}{ }_{B}^{D} \delta f\right) \\
& +(n+2 w-2)(n+2 w-4)(n+2 w-5)(n+2 w-7)( \\
& \left.-\frac{1}{10} n^{a} n^{b} n^{c} n^{d} \nabla_{a} \nabla_{b} \nabla_{c} \nabla_{d} f+\text { lots }\right)
\end{aligned}
$$

Figure $3 . \delta_{1,3} f$ for dimensions $n \geq 5$ and $f \in \mathcal{E}[w]$

of these cases, the formula in the figure expresses $\delta_{1,3}$ in terms of conformally invariant operators. To see this, note first that by [35], the (conformally invariant) Paneitz operator $P_{4}: \mathcal{E}[2-n / 2] \rightarrow \mathcal{E}[-2-n / 2]$ satisfies $P_{4}=-Y^{A} \square D_{A}$. Thus if $w=2-n / 2$ in Figure 3, then $\delta_{1,3}=3 P_{4}$. Similarly, if $w=5 / 2-n / 2$, then $-\bar{Y}^{A} \square \bar{D}_{A}$, in Figure 3, is the intrinsic Paneitz operator. On the other hand, $\square$ : $\mathcal{T}^{\Phi}[1-n / 2] \rightarrow \mathcal{T}^{\Phi}[-1-n / 2]$ is conformally invariant. The operators $L^{A B} \delta D_{A} D_{B}$, $N^{C} N^{D} W^{A}{ }_{C}{ }^{B}{ }_{D} D_{A} D_{B}$, and $L^{A}{ }_{C} L^{C B} D_{A} D_{B}$ in the figure have order less than four.

The $Q$-type curvature associated to $\delta_{1,3}$ is as follows:

$$
\begin{aligned}
& Q_{g}\left(\delta_{1,3}\right)=6(n-4)(n-2) H^{4}-36(n-4)(n-2) H^{2} n^{a} n^{b} \mathrm{P}_{a b} \\
& \quad-12(n-4)(n-2) H n^{a} n^{b} n^{c} \nabla_{a} \mathrm{P}_{b c} \\
& \quad-(n-4)(n-2) n^{a} n^{b} n^{c} n^{d} \nabla_{a} \nabla_{b} \mathrm{P}_{c d} \\
& \quad+(n-2) n^{a} n^{b} \Delta \mathrm{P}_{a b}+36(n-4) H^{2} \mathrm{~J}-3 \Delta \mathrm{J}+6 \mathrm{~J}^{2} \\
& \quad+36(n-4) H n^{a} \nabla_{a} \mathrm{~J}+2(n-6)(n-2) n^{a} n^{b} \mathrm{P}_{a c} \mathrm{P}_{b}^{c} \\
& \quad+6(n-4)(n-2) n^{a} n^{b} n^{c} n^{d} \mathrm{P}_{a b} \mathrm{P}_{c d}-12(n-4) n^{a} n^{b} \mathrm{P}_{a b} \mathrm{~J} \\
& \quad+(5 n-22) n^{a} n^{b} \nabla_{a} \nabla_{b} \mathrm{~J}-2(n-2) \mathrm{P}_{a b} \mathrm{P}^{a b}+2(n-2) n^{a} n^{b} \mathrm{P}_{c d} C_{a}{ }^{c} b
\end{aligned}
$$

8.4. Fifth-Order Operator. Finally, consider $\delta_{2,3}: \mathcal{E}[w] \rightarrow \overline{\mathcal{E}}[w-5]$. By (63), we see that

$$
\begin{aligned}
& (n+2 w-6) \delta_{2,3} f= \\
& \quad N^{A} N^{B} N^{C} N^{E} \delta D_{E}\left(D_{A} D_{B} D_{C} f-\frac{2}{n-4} X_{A} W_{B}{ }^{F} C^{G} D_{F} D_{G} f\right) .
\end{aligned}
$$




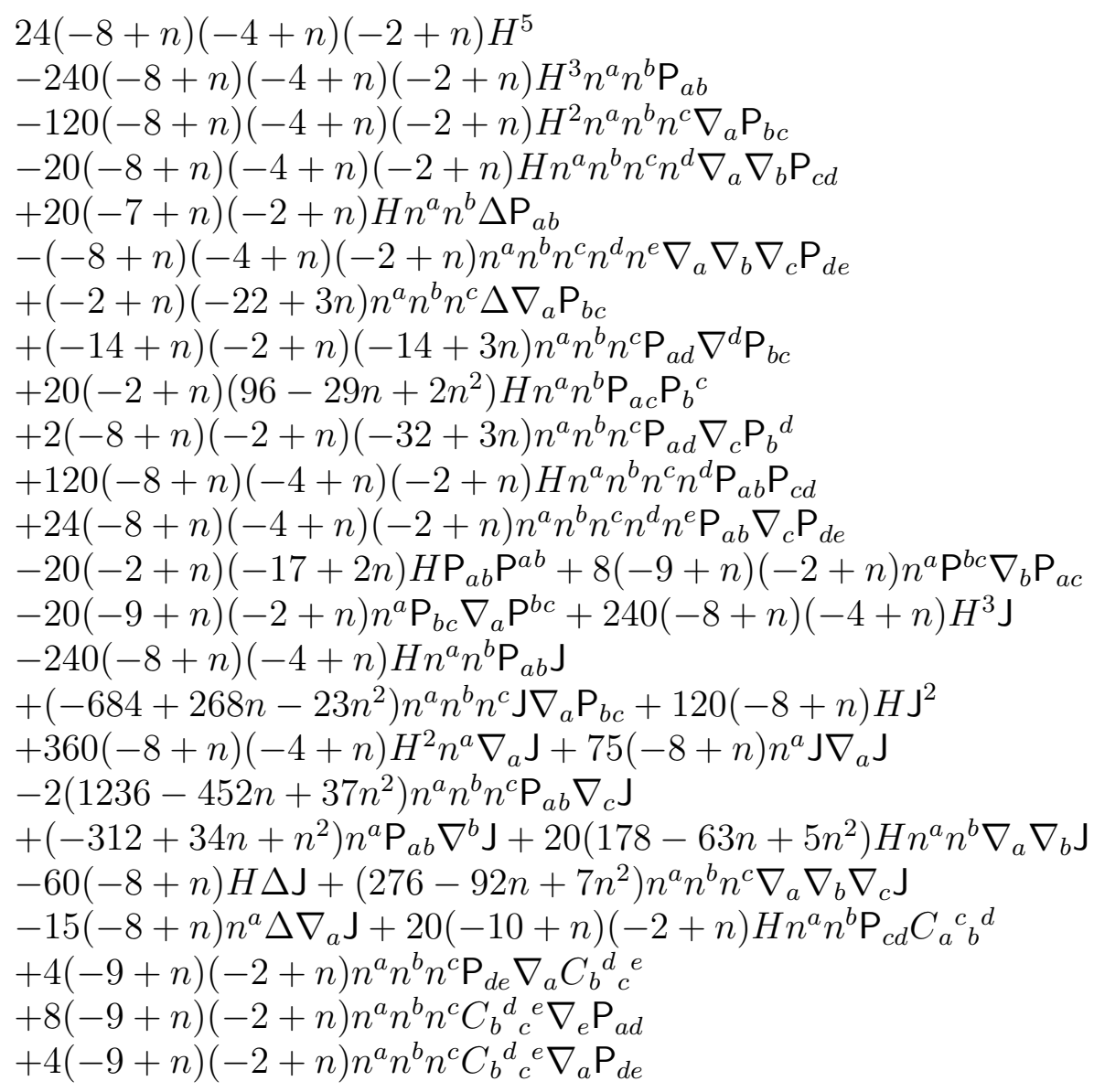

Figure $4 . Q_{g}\left(\delta_{2,3}\right)$

One may use (64) to express $\delta_{2,3} f$, in terms of $\nabla, \stackrel{o}{L}_{a b}, H$, the normal vector $n$, the curvature of a representative $g$ of the conformal structure, and $f$. By setting $w$ equal to $2-n / 2$ in the resulting expanded formula, one can show that $\delta_{2,3}=-60 \delta P_{4}$, where $P_{4}$ is the (conformally invariant) Paneitz operator. Similarly, for $f \in \mathcal{E}[1-n / 2]$, we have $\delta_{2,3} f=-5 \delta_{3} \square f$; note that in this context, $\square$ is conformally invariant. An expanded formula for $Q_{g}\left(\delta_{2,3}\right)$ appears in Figure 4 .

\section{FinAL REMARKS}

Here we mention some related points that we find very interesting. We only touch on these points however, as a full treatment lies beyond the scope of the current work.

9.1. Poincaré-Einstein manifolds. We recall the well-known notions of conformally compact and Poincaré-Einstein manifolds. For simplicity, we assume here that all relevant structures are orientable.

Definition 9.1. Let a compact manifold $M$ of dimension $n$ with boundary $\Sigma$ be given. Also let a Riemannian metric $g_{+}$on the interior, $M_{+}$, of $M$ be given, and 
suppose that for some nonnegative defining function $x$ for $\Sigma$, the metric $g=x^{2} g_{+}$ extends to a Riemannian metric on $M$. Then we say that $\left(M_{+}, g_{+}\right)$is conformally compact. The restriction of $g$ to $T \Sigma$ determines a conformal structure $\overline{\mathbf{c}}$ on $\Sigma$, and we say that $(\Sigma, \overline{\mathbf{c}})$ is the conformal infinity of $M_{+}$. Finally, suppose that the metric $g_{+}$on $M_{+}$is Einstein and that $\operatorname{Rc}^{g_{+}}=-(n-1) g_{+}$. Then we say that $M$ is a Poincaré-Einstein manifold.

In fact, we will assume, as above, that $M$, the defining function $x$, and $g$ are extended to a collar neighbourhood of $\Sigma$. The assumption that $x$ is nonnegative will only apply to points of the original manifold.

Conformally compact manifolds have, in particular, the structure of a conformal manifold with boundary. Thus all the results from the earlier sections apply to this setting. It is worth noting, however, that in the special case of a Poincaré-Einstein manifold, rather strong simplifications arise. (This is also true for manifolds which are asymptotically Einstein to sufficient order. We leave the details of this to the reader, but some related results are discussed in [19].) This stems from the following result.

Lemma 9.2. Let $\Sigma$ be the conformal infinity of a Poincaré-Einstein manifold $M$, and let $x$ be a nonnegative defining function for $\Sigma$ as in Definition 9.1. Let $\sigma=x \xi^{g}$, and let $I^{A}=\frac{1}{n} D^{A} \sigma$. Then $\left.I^{A}\right|_{\Sigma}=\left.N^{A}\right|_{\Sigma}$, and $I^{A}$ has the following properties on $M$ : (1) $I^{A}$ is parallel, (2) $I_{A} I^{A}=1$, and (3) the contraction of $I^{A}$ with any index of $W_{B C D E}$ is zero.

Proof. The fact that $\left.I^{A}\right|_{\Sigma}=\left.N^{A}\right|_{\Sigma}$ and properties (1) and (2) are proved in [31]. (See also [19] for a recent treatment.) Note that [31] and [19] use $d$ and $n$ to denote the dimensions of $M$ and $\Sigma$, respectively. By [35], $W_{B C D E}$ has Weyl tensor symmetries. Thus $W_{B C D E}=-W_{B C E D}$ and $W_{B C D E}=W_{D E B C}$. Since $I^{A}$ is parallel, property (3) follows from (12) and (14).

Moreover since $\left.I^{A}\right|_{\Sigma}=N^{A}$ is parallel along $\Sigma$, it follows that $\Sigma$ is totally umbilic [29]. The Einstein condition, i.e. the fact that $I^{A}$ is parallel, also forces other hypersurface invariants to vanish, cf. [19, 37]. We should thus expect the formulae for the boundary operators and associated curvatures to simplify. Indeed, we have the following:

Proposition 9.3. Let $\Sigma$ be the conformal infinity of a Poincaré-Einstein manifold M. Let $g$ and $n$ be as in Definition [9.1, and let $\mathbf{c}$ denote the conformal class associated to $g$. Let $j, k \in \mathbb{Z}_{>0}$ be given, and suppose that $n$ and $(M, \mathbf{c})$ satisfy Condition 5.9. Finally, let $P$ be as in Proposition 5.14. Then

$$
N^{A_{1}} \cdots N^{A_{k}} \delta_{j} P_{A_{1} \cdots A_{k}}=N^{A_{1}} \cdots N^{A_{k}} \delta_{j} D_{A_{1}} \ldots D_{A_{k}}
$$

along $\Sigma$.

Proof. From Lemma 9.2, it follows that

$$
N^{A_{1}} \cdots N^{A_{k}} \delta_{j} P_{A_{1} \cdots A_{k}}=\delta_{j} I^{A_{1}} \cdots I^{A_{k}} P_{A_{1} \cdots A_{k}}
$$

along $\Sigma$. But by Proposition 5.10, in every term of the tractor formula for $\mathcal{P}^{k}{ }_{A_{2} \cdots A_{k}}$, at least one of the indices $A_{2}, \ldots, A_{k}$ appears on a $W$. Thus (65) follows from (42) and Lemma 9.2 . 
The simplification expressed in (65) enables, in this setting, further refinements beyond Theorem 5.16. This will be taken up elsewhere. Meanwhile, inspired by Proposition 9.3, we now consider the very simple operator

$$
N^{A_{1}} \cdots N^{A_{k}} D_{A_{1}} \ldots D_{A_{k}}=I^{A_{1}} \ldots I^{A_{k}} D_{A_{1}} \ldots D_{A_{k}}
$$

along the boundary $\Sigma$ of a Poincaré-Einstein manifold. The right-hand side of (66) is defined not only along $\Sigma$, but on all of $M$ as well. Since $I$ is parallel, the right-hand side of (66) is simply $(I \cdot D)^{k}$. Here $I \cdot D=I^{A} D_{A}$. This idea was studied in [32] (to produce GJMS operators of the interior manifold) and in e.g. [36, 37]. These operators have a number of applications. For example, for any $m \in \mathbb{Z}_{>0}$,

$$
(I \cdot D)^{2 m}: \mathcal{E}\left[m-\frac{n-1}{2}\right] \rightarrow \mathcal{E}\left[-m-\frac{n-1}{2}\right]
$$

acts tangentially along $\Sigma$ [36, Theorem 4.1], and for suitable $m$ recovers nonzero multiples of the order-2 $m$ GJMS operators of the boundary [36, Theorem 4.5]. Here tangential is as defined in [36]. (In comparing with [36], note that with $I$ parallel it follows that $I^{2}:=I^{A} I_{A}$ is constant, and in the Poincaré-Einstein case this constant is non-zero.) From this one can, in an obvious way, find "factorisations" of the $I \cdot D$ powers at appropriate weights. For example, along $\Sigma$ and for $k \in \mathbb{Z}_{>0}$,

$$
(I \cdot D)^{2 m+k}=(I \cdot D)^{2 m} \circ(I \cdot D)^{k}: \mathcal{E}\left[m+k-\frac{n-1}{2}\right] \rightarrow \mathcal{E}\left[-m-\frac{n-1}{2}\right]
$$

evidently factors as a composition of a GJMS operator of $\Sigma$ applied to the restriction to $\Sigma$ of $(I \cdot D)^{k}$ acting on $\mathcal{E}[m+k-(n-1) / 2]$. Factorisations along these lines have been sought in the work [47] of Juhl. Again, further examples are beyond the scope of the current work, but we believe that these observations are of interest. In particular we believe that, in the setting of Poincaré-Einstein (or suitably asymptotically Poincaré-Einstein) manifolds, one may use these observations, together with the main techniques of the present paper, to construct simpler families of boundary operators that solve the problems 1.2 (and 1.3), and indeed yield rather stronger results. Again this will be taken up elsewhere.

9.2. Juhl's conjectures. Several of the results in the sections above solve problems closely related to (other) directions and conjectures in Juhl's monograph [47]. For example, the $T$-curvature pairs of Theorem 1.6, above, satisfy the "fundamental identity" that expression (1.10.4) in [47] would satisfy if the residue described there were to vanish. So the critical T-curvature pair of Theorem 1.6 provides a version of the objects sought at that point in [47]. (See also Chapter 6 of that work.)

The tractor families " $D_{K}^{\mathcal{T}}(M, \Sigma ; g ; \lambda)$ " of Definition 6.21.1 and Definition 6.21.2 in [47] are the operators denoted " $\delta_{K}$ " in Theorem 5.1 of [6]. In comparing with our discussion above, the reader should note that in [47], $n$ denotes $\operatorname{dim}(\Sigma)$, whereas here $n$ is $\operatorname{dim}(M)=\operatorname{dim}(\Sigma)+1$. Conjecture 1.10.1 of [47] (which is stated in more detail as Conjecture 6.21.3 of the same source) proposes that for case of $\operatorname{dim}(\Sigma)$ even, these operators admit a certain decomposition into an intrinsic Laplacian power part plus another part, termed an "extrinsic" part, and that this occurs in 
a manner compatible with corresponding $Q$-curvatures. As stated, that conjecture relies on Conjecture 6.21.1 of [47], which asserts that for $\operatorname{dim}(\Sigma)$ even, $\delta_{K}^{B G}$ is the zero operator on densities of weight $w=0$.

We do not see evidence that Conjecture 6.21.1 should hold in the curved setting if $\operatorname{dim}(\Sigma)$ is (even and) greater than 4 . However, any of the operators $\delta_{J, k}$, with $J+k=\operatorname{dim}(\Sigma)$, or either of the operators $\delta_{K}$ or $\delta_{K}^{0}$, with $K=\operatorname{dim}(\Sigma)$, provides a replacement for the operator $D_{\operatorname{dim}(\Sigma)}^{T}(\cdot, \Sigma ; g ; 0)$ sought in that conjecture, as $\delta_{K}$, $\delta_{K}^{0}$, and $\delta_{J, k}$ are all regular at weight 0 . One can use $\delta_{K}, \delta_{K}^{0}$, and $\delta_{J, k}$ to show that a variant of Juhl's Conjecture 6.21 .3 (or 1.10.1) does in fact hold. To see this, begin by supposing that $n \geq 5$ and that $n$ is odd. Let $\bar{P}_{n-1}$ denote the intrinsic order- $(n-1)$ GJMS operator on $\Sigma$ mapping $\overline{\mathcal{E}}[0]$ to $\overline{\mathcal{E}}[1-n]$. Let $\bar{B}_{n-1}(\bar{g})$ denote the Branson $Q$-curvature of $\Sigma$ associated to the metric $\bar{g}$ on $\Sigma$ induced by a representative $g$ of the conformal structure on $M$. View $\bar{B}_{n-1}(\bar{g})$ as a density of weight $1-n$. For a conformal metric $\widehat{g}=e^{2 \Upsilon} g$ on $M$,

$$
\bar{B}_{n-1}(\widehat{\bar{g}})=\bar{B}_{n-1}(\bar{g})+(-1)^{(n-1) / 2} \bar{P}_{n-1} \Upsilon \text {, }
$$

by (1.13) of [4]. The factor $(-1)^{(n-1) / 2}$ in (68) occurs because Branson's sign convention for $\Delta$ differs from ours.

With these preparations complete, we obtain at once the promised variant of Juhl's Conjecture 6.21.3.

Theorem 9.4. Suppose that $\operatorname{dim}(M)=n \geq 5$, and suppose that $n$ is odd. Let $\alpha \in \mathbb{R}$ be given, and let $K=n-1$. Let $\psi: \mathcal{E}[w] \rightarrow \overline{\mathcal{E}}[w-K]$ denote the operator $\delta_{K}$ of Lemma 5.4, as acting on densities, the operator $\delta_{K}^{0}$ of Theorem 5.12, or the operator $\delta_{J, k}$ of Theorem 5.16. (In the latter case, $J+k$ must equal $K$.) Then there exist natural conformally invariant differential operators $\mathbf{P}_{n-1}: \overline{\mathcal{E}}[0] \rightarrow \overline{\mathcal{E}}[1-n]$ and $P_{n-1}^{e}: \mathcal{E}[0] \rightarrow \overline{\mathcal{E}}[1-n]$ and natural sections $\mathbf{Q}_{n-1}(\bar{g})$ and $Q_{n-1}^{e}(g)$ of $\overline{\mathcal{E}}[1-n]$ and $\left.\mathcal{E}[1-n]\right|_{\Sigma}$, respectively, with the following properties. First, for the operator $\psi: \mathcal{E}[0] \rightarrow \overline{\mathcal{E}}[1-n]$, we have $\alpha \psi=\mathbf{P}_{n-1}+P_{n-1}^{e}$. Also,

$$
(-1)^{(n-1) / 2} Q_{g}(\alpha \psi)=\mathbf{Q}_{n-1}(\bar{g})+Q_{n-1}^{e}(g) .
$$

Here $\mathbf{P}_{n-1}$ has leading term $\bar{\Delta}^{(n-1) / 2}$, and $Q_{g}(\alpha \psi)$ is as in Definition 7.5. We view $Q_{g}(\alpha \psi)$ as a section of $\left.\mathcal{E}[1-n]\right|_{\Sigma}$. Finally, suppose that $\widehat{g}=e^{2 \Upsilon} g$. Then

$$
\mathbf{Q}_{n-1}(\widehat{\bar{g}})=\mathbf{Q}_{n-1}(\bar{g})+(-1)^{(n-1) / 2} \mathbf{P}_{n-1} \Upsilon \text {, }
$$

and

$$
Q_{n-1}^{e}(\widehat{g})=Q_{n-1}^{e}(g)+(-1)^{(n-1) / 2} P_{n-1}^{e} \Upsilon .
$$

Remark 9.5. We do not claim that $\psi, \mathbf{P}_{n-1}, P_{n-1}^{e}, \mathbf{Q}_{n-1}(\bar{g})$, or $Q_{n-1}^{e}(g)$ is uniquely determined, but see Remark 5.11.

Proof of Theorem 9.4. Let $\bar{P}_{n-1}$ and $\bar{B}_{n-1}(\bar{g})$ be as above. Let $P_{n-1}^{e}=\alpha \psi-\bar{P}_{n-1}$, and let $\mathbf{P}_{n-1}=\bar{P}_{n-1}$. Let $\mathbf{Q}_{n-1}(\bar{g})=\bar{B}_{n-1}(\bar{g})$, and let

$$
Q_{n-1}^{e}(g)=(-1)^{(n-1) / 2} Q_{g}(\alpha \psi)-\mathbf{Q}_{n-1}(\bar{g}) .
$$

The transformation rules given in (69) and (170) then follow from (68) and Proposition 7.7 . 


$$
\left[r_{a b}\right]=\left[\begin{array}{c|c}
\left.g_{a b}\right|_{p} & O_{m \times d} \\
\hline O_{d \times m} & I_{d \times d}
\end{array}\right], \quad\left[r^{a b}\right]=\left[\begin{array}{c|c}
\left.g^{a b}\right|_{p} & O_{m \times d} \\
\hline O_{d \times m} & I_{d \times d}
\end{array}\right]
$$

FiguRE 5. The component matrices of $r$ and its inverse at a point $(p, q) \in M \times \mathbb{R}^{d}$

As stated Juhl's Conjecture 6.21.3 allows for the freedom to choose $\alpha$ in Theorem 9.4. However it is natural to fix this to the special value $\alpha=\alpha_{0} \neq 0$ so that when specialised to the case that $\left(M, g_{+}\right)$is the Poincaré ball metric, with $\Sigma$ the boundary at infinity (i.e. the conformally flat model for Poincaré-Einstein geometry), then $P_{n-1}^{e}$ is zero. For the case of $\psi=\delta_{K}$ the existence of $\alpha_{0}$ follows directly from the discussion surrounding (67). More generally it follows from the uniqueness of the intertwinors in this case.

Finally we mention that the role of extrinsic geometry in generalised notions of $Q$-curvature (and related " $T$-curvatures" as associated transgression curvatures) is explored in [38, 39].

\section{Appendix A. Proof of Proposition 4.2}

All of the expressions in this proof will carry a weight of zero. Let $d=m^{\prime}-m$, and let $M^{\prime}=M \times \mathbb{R}^{d}$. Also let $\Sigma^{\prime}=\Sigma \times \mathbb{R}^{d}$. Let $g_{E}$ denote the Euclidean metric on $\mathbb{R}^{d}$, and let $r$ denote the product metric $g \oplus g_{E}$ on $M^{\prime}$. One key to our proof is the fact that the operator $\psi$ is given by the same universal symbolic formula on $M$ and on $M^{\prime}$. Our strategy will be to relate expressions on $\left(M^{\prime}, r\right)$ to their counterparts on $(M, g)$.

Let $\left(y^{1}, \ldots, y^{d}\right)$ denote the standard coordinates on $\mathbb{R}^{d}$. Let $U$ be any open subset of $M$ on which a local coordinate system $\left(x^{1}, \ldots, x^{m}\right)$ is defined, and suppose there is a local defining function $t$ for $\Sigma$ on $U$. Note that $\left(z^{1}, \ldots, z^{m^{\prime}}\right)=$ $\left(x^{1}, \ldots, x^{m}, y^{1}, \ldots, y^{d}\right)$ is a local coordinate system for $M^{\prime}$ on $U \times \mathbb{R}^{d}$. Let $\Gamma_{a b}{ }^{c}$ and $\Gamma_{a b}^{\prime c}$ denote the Christoffel symbols of $g$ and $r$ in the $\left(x^{1}, \ldots, x^{m}\right)$ and $\left(z^{1}, \ldots, z^{m^{\prime}}\right)$ coordinate systems, respectively.

Figure 5 gives the components of $r$ and its inverse in the $\left(z^{1}, \ldots, z^{m^{\prime}}\right)$ coordinate system at a point $(p, q) \in M^{\prime}$. In this figure, $\left.g_{a b}\right|_{p}$ and $\left.g^{a b}\right|_{p}$ denote the $m \times m$ component matrices of $g$ and its inverse, respectively, at $p, I_{d \times d}$ is the $d \times d$ identity matrix, and $O_{m \times d}$ and $O_{d \times m}$ are the zero matrices of the indicated dimensions.

Note that $\Gamma_{a b}^{\prime}{ }^{c}=\frac{1}{2} r^{c d}\left(\partial_{a} r_{b d}+\partial_{b} r_{a d}-\partial_{d} r_{a b}\right)$. Thus if $\{a, b, c\}$ is not a subset of $\{1, \ldots, m\}$, one can use the formulae in Figure 5 to show that $\Gamma_{a b}^{\prime}{ }^{c}=0$ on $U \times \mathbb{R}^{d}$. On the other hand, if $\{a, b, c\}$ is a subset of $\{1, \ldots, m\}$, then for all $(p, q) \in U \times \mathbb{R}^{d}$, we have $\left.\Gamma_{a b}^{\prime}{ }^{c}\right|_{(p, q)}=\left.\Gamma_{a b}{ }^{c}\right|_{p}$. For all $\{a, b, c\} \subseteq\left\{1, \ldots, m^{\prime}\right\}, \Gamma_{a b}^{\prime}{ }^{c}$ is independent of $z^{m+1}, \ldots$, and $z^{m^{\prime}}$.

Let $\left(R^{\prime}\right)_{a b}{ }^{c} d$ denote the components of the Riemannian curvature tensor of $r$. Then

$$
\left(R^{\prime}\right)_{a b d}^{c}=\frac{\partial}{\partial z^{a}} \Gamma_{b d}^{\prime c}-\frac{\partial}{\partial z^{b}} \Gamma_{a d}^{\prime c}+\Gamma_{b d}^{\prime e} \Gamma_{a e}^{\prime c}-\Gamma_{a d}^{\prime e} \Gamma_{b e}^{\prime c}
$$


Now let $(p, q) \in U \times \mathbb{R}^{d}$ be given. If $\{a, b, c, d\} \subseteq\{1, \ldots, m\}$, then $\left.\left(R^{\prime}\right)_{a b}{ }^{c}\right|_{(p, q)}=$ $\left.R_{a b d}^{c}\right|_{p}$. Otherwise, $\left.\left(R^{\prime}\right)_{a b}^{c}{ }_{d}\right|_{(p, q)}=0$.

We will now construct a unit conormal field for $\Sigma^{\prime}$. Let $\pi: U \times \mathbb{R}^{d} \rightarrow U$ be the natural projection, and let $t^{\prime}$ denote the lift (pullback) of $t$ along $\pi$. Consider any given $(p, q) \in U \times \mathbb{R}^{d}$. Note that $\left.\left(d t^{\prime}\right)\right|_{(p, q)}=\left.\left.\frac{\partial t}{\partial x^{i}}\right|_{p} d x^{i}\right|_{(p, q)}$ and that $t^{\prime}(p, q)=0$ if and only if $p \in \Sigma$. Thus $t^{\prime}$ is a local defining function for $\Sigma^{\prime}$ on $U \times \mathbb{R}^{d}$. The unit conormal field for $\Sigma^{\prime}$ on $\Sigma^{\prime} \cap\left(U \times \mathbb{R}^{d}\right)$ is given by $n_{a}^{\prime}=\frac{d t^{\prime}}{\left|d t^{\prime}\right|_{r}}$. If we consider Figure 5, we find that $\left|d t^{\prime}\right|_{r}$, evaluated at $(p, q)$, is equal to $|d t|_{g}$, evaluated at $p$. Now let $a \in\left\{1, \ldots, m^{\prime}\right\}$ be given, and let $n_{a}^{\prime}$ denote the component of the unit conormal field for $\Sigma^{\prime}$ corresponding to $a$. If $m+1 \leq a \leq m^{\prime}$, then $\left.n_{a}^{\prime}\right|_{(p, q)}=0$. If $1 \leq a \leq m$, then $\left.n_{a}^{\prime}\right|_{(p, q)}=\left.n_{a}\right|_{p}$; here $\left.n_{a}\right|_{p}$ denotes the component of $n_{a}$ at $p$.

Let $G^{\prime}$ denote the modified mean curvature associated to $M^{\prime}$ and $\Sigma^{\prime}$, as defined in Section 3.1, above. Also let $\nabla^{\prime}$ denote the Levi-Civita connection of $r$ on $M^{\prime}$. Then $G^{\prime}=r^{a b} \nabla_{a}^{\prime} n_{b}^{\prime}$ on $U \times \mathbb{R}^{d}$, and for any $(p, q) \in U \times \mathbb{R}^{d}$, we have $\left.G^{\prime}\right|_{(p, q)}=\left.r^{a b}\left(\left(\partial / \partial z^{a}\right) n_{b}^{\prime}-\Gamma_{a b}^{\prime}{ }^{c} n_{c}^{\prime}\right)\right|_{(p, q)}$. We may assume here that $a, b$, and $c$ are summed from 1 to $m$ only. Thus

$$
\left.G^{\prime}\right|_{(p, q)}=\left.g^{a b}\left(\frac{\partial}{\partial x^{a}} n_{b}-\Gamma_{a b}{ }^{c} n_{c}\right)\right|_{p}=\left.G\right|_{p} .
$$

Next, let $V^{\prime}$ denote the lift of $V$ along $\pi$. Also let $\beta \in \mathbb{Z}_{>0}$ and $(p, q) \in U \times \mathbb{R}^{d}$ be given, and consider the following component expressions:

$$
\left.\left(\nabla_{a_{1}}^{\prime} \ldots \nabla_{a_{\beta}}^{\prime} V^{\prime}\right)\right|_{(p, q)} \text { and }\left.\left(\nabla_{a_{1}}^{\prime} \ldots \nabla_{a_{\beta}}^{\prime}\left(R^{\prime}\right)_{i j}{ }^{k} \ell\right)\right|_{(p, q)} .
$$

We may symbolically expand both of these and express them in terms of the Christoffel symbols $\Gamma_{i j}^{\prime k}$ and partial derivatives of $V^{\prime}, \Gamma_{i j}^{\prime}{ }^{k}$ and $\left(R^{\prime}\right)_{i j}{ }^{k} \ell$. (See Section 2.5 of [60].) Let $\mathcal{P}_{1}$ and $\mathcal{P}_{2}$ denote the expressions that result from the expansion of the respective expressions in (71) in this way. Note that $V^{\prime}, \Gamma_{i j}^{\prime k}$, and $\left(R^{\prime}\right)_{i j}{ }^{k} \ell$ are independent of $q$. Also recall that $\left.\Gamma_{a b}^{\prime}{ }^{c}\right|_{(p, q)}$ vanishes whenever $\{a, b, c\}$ is not a subset of $\{1, \ldots, m\}$. Similarly, $\left.\left(R^{\prime}\right)_{a b}{ }^{c} d\right|_{(p, q)}$ vanishes if $\{a, b, c, d\}$ is not a subset of $\{1, \ldots, m\}$. We may thus assume that all indices in $\mathcal{P}_{1}$ and $\mathcal{P}_{2}$ take values in $\{1, \ldots, m\}$ only.

Now let $p \in \Sigma$ and $q \in \mathbb{R}^{d}$ be given. We may assume that $p \in U$. We claim that $(\psi V)(p)=\left(\psi V^{\prime}\right)(p, q)$. To see this, begin by expanding the symbolic formula for $\left(\psi V^{\prime}\right)(p, q)$ in the way in which we expanded the two expressions in (71), above. In the resulting expansion, we may assume that all indices are summed from 1 to $m$ only. Next, replace all occurrences of $\left(R^{\prime}\right)_{a b}{ }^{c}{ }_{(p, q)},\left.\Gamma_{a b}^{\prime}{ }^{c}\right|_{(p, q)},\left.G^{\prime}\right|_{(p, q)}$, $\left.n_{a}^{\prime}\right|_{(p, q)},\left.r_{a b}\right|_{(p, q)},\left.r^{a b}\right|_{(p, q)}, V^{\prime}(p, q)$, and $\left.\frac{\partial}{\partial z^{i}}\right|_{(p, q)}$ with $\left.R_{a b}{ }^{c}\right|_{p},\left.\Gamma_{a b}{ }^{c}\right|_{p},\left.G\right|_{p},\left.n_{a}\right|_{p},\left.g_{a b}\right|_{p}$, $\left.g^{a b}\right|_{p}, V(p)$, and $\left.\frac{\partial}{\partial x^{i}}\right|_{p}$, respectively. The resulting expression is $(\psi V)(p)$. If we let $p^{\prime}=(p, q)$, then $p^{\prime}$ satisfies (25).

\section{REFERENCES}

[1] S. Agmon, A. Douglis, L. Nirenberg: Estimates near the boundary for solutions of elliptic partial differential equations satisfying general boundary conditions. II. Comm. Pure Appl. Math. 17 (1964), 35-92. 
[2] M. Atiyah, R. Bott, V.K. Patodi: On the heat equation and the index theorem. Invent. Math. 19 (1973), 279-330.

[3] T.N. Bailey, M.G. Eastwood, A.R. Gover: Thomas's structure bundle for conformal, projective and related structures. Rocky Mountain J. Math. 24 (1994), no. 4, 1191-1217.

[4] T.P. Branson: Sharp inequalities, the functional determinant, and the complementary series. Trans. Amer. Math. Soc. 347 (1995), no. 10, 3671-3742.

[5] T.P. Branson: Spectral theory of invariant operators, sharp inequalities, and representation theory. In: The Proceedings of the 16th Winter School "Geometry and Physics" (Srní, 1996). Rend. Circ. Mat. Palermo (2) Suppl. No. 46 (1997), 29-54.

[6] T.P. Branson, A.R. Gover: Conformally invariant non-local operators. Pacific J. Math. 201 (2001), no. 1, 19-60.

[7] L. Caffarelli, L. Silvestre: An extension problem related to the fractional Laplacian. Comm. Partial Differential Equations 32 (2007), no. 7-9, 1245-1260.

[8] D.M.J. Calderbank: Möbius structures and two-dimensional Einstein-Weyl geometry. $J$. Reine Angew. Math. 504 (1998), 37-53.

[9] A. Cap, A.R. Gover: Standard tractors and the conformal ambient metric construction. Ann. Global Anal. Geom. 24 (2003), no. 3, 231-259.

[10] A. Čap, A.R. Gover: Tractor calculi for parabolic geometries. Trans. Amer. Math. Soc. 354 (2002), no. 4, 1511-1548.

[11] J.S. Case: Boundary operators associated to the Paneitz operator. Indiana Univ. Math. J., in press. arXiv:1509.08342.

[12] J.S. Case, S.-Y.A. Chang: On fractional GJMS operators. Comm. Pure Appl. Math. 69 (2016), no. 6, 1017-1061.

[13] G. Catino, C.B. Ndiaye: Integral pinching results for manifolds with boundary. Ann. Sc. Norm. Super. Pisa Cl. Sci. (5) 9 (2010), no. 4, 785-813.

[14] S.-Y.A. Chang: Conformal invariants and partial differential equations. Bull. Amer. Math. Soc. (N.S.) 42 (2005), no. 3, 365-393.

[15] S.-Y.A. Chang, M. González: Fractional Laplacian in conformal geometry. Adv. Math. 226 (2011), no. 2, 1410-1432.

[16] S.-Y.A. Chang, J. Qing: The zeta functional determinants on manifolds with boundary. I. The formula. J. Funct. Anal. 147 (1997), no. 2, 327-362.

[17] P. Cherrier: Problèmes de Neumann non linéaires sur les variétés riemanniennes. J. Funct. Anal. 57 (1984), no. 2, 154-206.

[18] J.-L. Clerc: Another approach to Juhl's conformally covariant differential operators from $S^{n}$ to $S^{n-1}$. SIGMA Symmetry Integrability Geom. Methods Appl. 13 (2017), Paper 026, $18 \mathrm{pp}$.

[19] S. Curry, A.R. Gover: An introduction to conformal geometry and tractor calculus, with a view to applications in general relativity. In: Asymptotic Analysis in General Relativity. Ed. T. Daudé, D. Häfner, J.-P. Nicolas. London Mathematical Society Lecture Note Series, 443. Cambridge University Press, Cambridge, in press. arXiv:1412.7559.

[20] Z. Djadli, A. Malchiodi: Existence of conformal metrics with constant $Q$-curvature. Ann. of Math. (2) 168 (2008), no. 3, 813-858.

[21] M.G. Eastwood: Notes on conformal differential geometry. In: The Proceedings of the 15th Winter School "Geometry and Physics" (Srní, 1995). Rend. Circ. Mat. Palermo (2) Suppl. No. 43 (1996), 57-76.

[22] M.G. Eastwood, J. Slovak: Semiholonomic Verma modules. J. Algebra 197 (1997), no. 2, 424-448.

[23] J.F. Escobar: Conformal deformation of a Riemannian metric to a scalar flat metric with constant mean curvature on the boundary. Ann. of Math. (2) 136 (1992), no. 1, 1-50. Addendum in Ann. of Math. (2) 139 (1994), no. 3, 749-750.

[24] J.F. Escobar: The Yamabe problem on manifolds with boundary. J. Differential Geom. 35 (1992), no. 1, 21-84. 
[25] C. Fefferman, C.R. Graham: The ambient metric. Annals of Mathematics Studies, 178. Princeton University Press, Princeton, NJ, 2012. x+113 pp.

[26] C. Fefferman, C.R. Graham: Conformal invariants. In: Elie Cartan et les mathématiques d'aujourd'hui. Astérisque 1985, hors série. Société Mathématique de France, Paris, 95-116.

[27] C. Fefferman, C.R. Graham: Q-curvature and Poincaré metrics. Math. Res. Lett. 9 (2002), no. 2-3, 139-151.

[28] M. Fischmann, A. Juhl, P. Somberg: Conformal symmetry breaking differential operators on differential forms. arXiv:1605.04517.

[29] A.R. Gover: Almost Einstein and Poincaré-Einstein manifolds in Riemannian signature. $J$. Geom. Phys. 60 (2010), no. 2, 182-204.

[30] A.R. Gover: Aspects of parabolic invariant theory. In: The 18th Winter School "Geometry and Physics" (Srní 1998). Rend. Circ. Mat. Palermo (2) Suppl. No. 59 (1999), 25-47.

[31] A.R. Gover: Conformal Dirichlet-Neumann maps and Poincaré-Einstein manifolds. SIGMA Symmetry Integrability Geom. Methods Appl. 3 (2007), Paper 100, 21 pp.

[32] A.R. Gover: Laplacian operators and $Q$-curvature on conformally Einstein manifolds. Math. Ann. 336 (2006), no. 2, 311-334.

[33] A.R. Gover, K. Hirachi: Conformally invariant powers of the Laplacian-a complete nonexistence theorem. J. Amer. Math. Soc. 17 (2004), no. 2, 389-405.

[34] A.R. Gover, E. Latini, A. Waldron: Poincaré-Einstein holography for forms via conformal geometry in the bulk. Mem. Amer. Math. Soc. 235 (2015), no. 1106, vi+95 pp.

[35] A.R. Gover, L.J. Peterson: Conformally invariant powers of the Laplacian, $Q$-curvature, and tractor calculus. Comm. Math. Phys. 235 (2003), no. 2, 339-378.

[36] A.R. Gover, A. Waldron: Boundary calculus for conformally compact manifolds. Indiana Univ. Math. J. 63 (2014), no. 1, 119-163.

[37] A.R. Gover, A. Waldron: Conformal hypersurface geometry via a boundary LoewnerNirenberg-Yamabe problem. arXiv:1506.02723.

[38] A.R. Gover, A. Waldron: Renormalized volume. Comm. Math. Phys. 354 (2017), no. 3, $1205-1244$.

[39] A.R. Gover, A. Waldron: Renormalized Volumes with Boundary, arXiv:1611.08345.

[40] C.R. Graham: Conformally invariant powers of the Laplacian, II: Nonexistence. J. London Math. Soc. (2) 46 (1992), no. 3, 566-576.

[41] C.R. Graham, R. Jenne, L.J. Mason, G.A. Sparling: Conformally invariant powers of the Laplacian, I: Existence. J. London Math. Soc. (2) 46 (1992), no. 3, 557-565.

[42] C.R. Graham, M. Zworski: Scattering matrix in conformal geometry. Invent. Math. 152 (2003), no. 1, 89-118.

[43] D.H. Grant: A Conformally invariant third order Neumann-Type operator for hypersurfaces. M.S. thesis, University of Auckland, 2003. vi+70 pp.

[44] G. Grubb: Functional calculus of pseudodifferential boundary problems. Second edition. Progress in Mathematics, 65. Birkhäuser Boston, Inc., Boston, MA, 1996. x+522 pp.

[45] C. Guillarmou, L. Guillopé: The determinant of the Dirichlet-to-Neumann map for surfaces with boundary. Int. Math. Res. Not. IMRN 2007, no. 22, Art. ID rnm099, 26 pp.

[46] L. Hörmander: The analysis of linear partial differential operators. III. Pseudodifferential operators. Grundlehren der Mathematischen Wissenschaften, 274. Springer-Verlag, Berlin, 1985. viii $+525 \mathrm{pp}$.

[47] A. Juhl: Families of conformally covariant differential operators, $Q$-curvature and holography. Progress in Mathematics, 275. Birkäuser Verlag, Basel, 2009. xiv+488 pp.

[48] T. Kobayashi, T. Kubo, M. Pevzner: Conformal symmetry breaking operators for differential forms on spheres. Lecture Notes in Mathematics, 2170. Springer, Singapore, 2016. ix +192 pp.

[49] T. Kobayashi, M. Pevzner: Differential symmetry breaking operators: I. General theory and F-method. Selecta Math. (N.S.) 22 (2016), no. 2, 801-845. 
[50] T. Kobayashi, B. Speh: Symmetry breaking for representations of rank one orthogonal groups. Mem. Amer. Math. Soc. 238 (2015), no. 1126, v+110 pp.

[51] J.M. Lee: Ricci software package. http://www.math.washington.edu/ lee.

[52] J. Maldacena: The large $N$ limit of superconformal field theories and supergravity. $A d v$. Theor. Math. Phys. 2 (1998), no. 2, 231-252.

[53] C.B. Ndiaye: Conformal metrics with constant $Q$-curvature for manifolds with boundary. Comm. Anal. Geom. 16 (2008), no. 5, 1049-1124.

[54] C.B. Ndiaye: Constant T-curvature conformal metrics on 4-manifolds with boundary. Pacific J. Math. 240 (2009), no. 1, 151-184.

[55] R.M. Schoen: Conformal deformation of a Riemannian metric to constant scalar curvature. J. Differential Geom. 20 (1984), no. 2, 479-495.

[56] M. Spivak: A Comprehensive Introduction to Differential Geometry, Vol. III. Third edition. Publish or Perish, Inc., Houston, 1999. ix +314 pp.

[57] R. Stafford: Tractor Calculus and Invariants for Conformal Sub-Manifolds. M.S. thesis, University of Auckland, 2006. iv+48 pp.

[58] P. Stredder: Natural differential operators on Riemannian manifolds and representations of the orthogonal and special orthogonal groups. J. Differential Geometry 10 (1975), no. 4, $647-660$.

[59] L. Sun, J. Xiong: Classification theorems for solutions of higher order boundary conformally invariant problems, I. J. Funct. Anal. 271 (2016), no. 12, 3727-3764.

[60] J.L. Synge, A. Schild: Tensor Calculus. Dover Publications, Inc., New York, 1978. ix+324 pp.

[61] E. Witten: A new proof of the positive energy theorem. Comm. Math. Phys. 80 (1981), no. 3, 381-402.

[62] Wolfram Research: Mathematica computer software. Champaign, IL, USA.

Department of Mathematics, The University of Auckland, Private Bag 92019, Auckland 1142, New Zealand

E-mail address: r.gover@auckland.ac.nz

Department of Mathematics, The University of North Dakota, 101 Cornell Street Stop 8376, Grand Forks, ND 58202-8376, USA

E-mail address: lawrence.peterson@email.und.edu 